\title{
Data Repository Items
}

\section{BLUE RIDGE, COLORADO, QUARTZITE SUCCESSION}

\section{Rock descriptions}

The Proterozoic metasedimentary succession and crystalline basement rocks exposed along Blue Ridge were mapped and described by Reuss (1974), and the following unit descriptions are summarized largely from his work with additional data from the UNM Advanced Field Camp. Basement exposures are dominated by weakly foliated to locally mylonitic, coarse-grained granodiorite of the Paleoproterozoic Twin Mountain batholith (ca. 1705 Ma; Bickford et al., 1989) but also locally include

feldspathic biotite schist and gneiss. Basement granitoids are overlain by a 15-25 m thick layer of quartzite pebble conglomerate on the northern side of the metasedimentary succession (Figs. 2A and 2D). Quartzite pebbles are $0.5-2.0 \mathrm{~cm}$ in size, and the surrounding matrix consists of quartz, muscovite, and well-rounded zircon up to $0.1 \mathrm{~mm}$ in size. The lower 5-10 m of conglomerate also contain up to $20 \%$ microcline crystals that are $0.5-2.0 \mathrm{~cm}$ in size and contain numerous quartz and muscovite inclusions and black tourmaline veinlets. These clasts are interpreted to represent igneous material derived from the underlying granitoid basement. Between the conglomerate and basement granitoid rocks is a thin (5-10 m), poorly-exposed zone of phyllitic material that locally contains large granitic clasts (Fig. 2D). This zone is interpreted to be a regolith that represents part of the underlying granodiorite that was sub-aerially exposed and heavily weathered prior to deposition of the conglomerate. 
The quartzite pebble conglomerate is overlain by quartzite and schist that are exposed in eight northeast-trending, subvertical layers ranging in thickness from 15 to $350 \mathrm{~m}$ (Fig. 2A). The contact between conglomerate and quartzite ranges from sharp to gradational, and isolated lenses of conglomerate occur within the upper units of the quartzite. Based on the gradational stratigraphic progression from highly-weathered granitoid basement to basal conglomerate to quartzite and schist, the basal conglomerate and, therefore, the entire metasedimentary succession, is interpreted to be in unconformable depositional contact with underlying plutonic basement rocks. Quartzite is relatively pure (80-95\% quartz with $1-10 \%$ muscovite), and individual quartz grains range in size from 0.5 to $5.0 \mathrm{~mm}$ and are strongly recrystallized with interlocking, sutured grain boundaries. Tabular cross-bedding is well preserved (Fig. 2C) with individual bed thicknesses between 0.1 and $0.4 \mathrm{~m}$ thick, and paleocurrent analysis corrected for deformation suggests a dominant current direction from the north-northeast. Oscillation ripple marks are also locally preserved and have an average amplitude and wavelength of $0.5-1.5 \mathrm{~cm}$ and $2-5 \mathrm{~cm}$, respectively. Schist layers are dominated by quartz, biotite, and muscovite but also presently contain a variety of metamorphic minerals including garnet + sillimanite + andalusite + staurolite \pm cordierite \pm actinolite. Interlayered beds of quartzite and/or quartz pebble conglomerate are common.

\section{Structural relationships}

After deposition, the protoliths of the metasedimentary succession experienced two major phases of deformation and metamorphism. The first phase $\left(D_{1} / M_{1}\right)$ involved development of a bedding-parallel foliation accompanied by growth and/or 
recrystallization of biotite, muscovite, staurolite, and locally cordierite (Reuss, 1974). The second phase of deformation $\left(\mathrm{D}_{2}\right)$ produced the 4-km-wavelength Gooseberry Gulch syncline (Fig. 2B), the dominant structure at Blue Ridge (Fig. 2A). $\mathrm{D}_{2}$ folding was accompanied by amphibolite-facies metamorphism $\left(\mathrm{M}_{2}\right)$ and local development of a subvertical, axial-planar foliation $\left(\mathrm{S}_{2}\right)$. It is difficult to determine whether or not basement granitoids were folded with the overlying succession. The southern limb of the syncline is cut by a ductile shear zone that juxtaposes steeply-dipping quartzite and schist against strongly-deformed, locally-mylonitic granodiorite, omitting nearly $1 \mathrm{~km}$ of stratigraphic thickness from the lower part of the metasedimentary succession (Fig. 2B; Reuss, 1974). Deformation is most intense within 5-10 m of the granodiorite/quartzite contact and grades into weakly-foliated granodiorite across a distance of 20-30 m. The shear zone fabric is subparallel with $S_{2}$ in the quartzite succession and, thus, is interpreted to have formed during the late stages of $D_{2}$ shortening. The folded succession is also cut by a series of 60-180 m left-lateral, en-echelon folds that postdate development of the main syncline. These folds are interpreted to have formed during the late stages of $\mathrm{D}_{2}$ or during a later deformation event $\left(\mathrm{D}_{3}\right.$; Reuss, 1974).

\section{REFERENCES}

Bickford, M.E., Cullers, R.L., Shuster, R.D., Premo, W.R., and Van Schmus, W.R., 1989, $\mathrm{U}-\mathrm{Pb}$ zircon geochronology of Proterozoic and Cambrian plutons in the Wet Mountains and southern Front Range, Colorado, in Grambling, J. A., and Tewksbury, B. J., Proterozoic geology of the southern Rocky Mountains: Boulder, Colorado, Geological Society of America Special Paper 235, p. 49-64. 
Krogh, T.E., 1982, Improved accuracy of U-Pb zircon ages by the creation of more concordant systems using an abrasion technique: Geochimica et Cosmochimica Acta, v. 46, p. 637-649.

Reuss, R.L., 1974, Precambrian quartzite-schist succession in Wilson Park, Fremont County, Colorado: Mountain Geologist, v. 11, p. 45-58.

Stacey, J.S., and Kramers, J.D., 1975, Approximation of terrestrial lead isotopic evolution by a two-stage model: Earth and Planetary Science Letters, v. 26, p. 207-221. 
Table DR1. U-Pb Isotopic Data and Ages for Samples from Blue Ridge, Colorado

\begin{tabular}{|c|c|c|c|c|c|c|c|c|c|c|c|}
\hline \multirow{3}{*}{ Fraction } & \multirow{2}{*}{$\begin{array}{c}\text { Weight } \\
{[\mathrm{mg}]}\end{array}$} & Concentration & \multicolumn{2}{|c|}{ Measured } & \multicolumn{4}{|c|}{ *Corrected Atomic Ratios } & \multicolumn{3}{|c|}{ Ages (Ma) } \\
\hline & & $\mathrm{U} \quad \mathrm{Pb}^{\mathrm{R}}$ & Common & ${ }^{206} \mathrm{~Pb}$ & ${ }^{208} \mathrm{~Pb}$ & ${ }^{206} \mathrm{~Pb}$ & ${ }^{207} \mathrm{~Pb}$ & ${ }^{207} \mathrm{~Pb}$ & ${ }^{206} \mathrm{~Pb}$ & ${ }^{207} \mathrm{~Pb}$ & ${ }^{207} \mathrm{~Pb}$ \\
\hline & & [ppm] & $\mathrm{Pb}^{\mathrm{T}}[\mathrm{pg}]$ & ${ }^{204} \mathrm{~Pb}$ & ${ }^{206} \mathrm{~Pb}$ & ${ }^{238} \mathrm{U}$ & ${ }^{235} \mathrm{U}$ & ${ }^{206} \mathrm{~Pb}$ & $\overline{{ }^{238} \mathrm{U}}$ & $\overline{{ }^{235} \mathrm{U}}$ & ${ }^{206} \mathrm{~Pb}$ \\
\hline
\end{tabular}

\section{Foliated granodiorite (J01-BR3; Lat $\mathrm{N} 38^{\circ} 35.041^{\prime}$, Long $\mathrm{W} 105^{\circ} \mathbf{1 8 . 2 5 8}^{\prime}$ )}

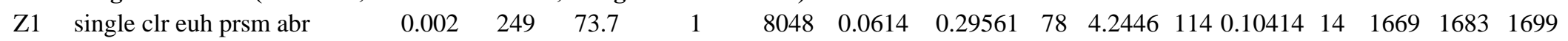

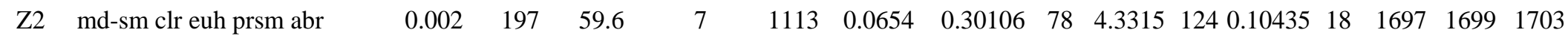

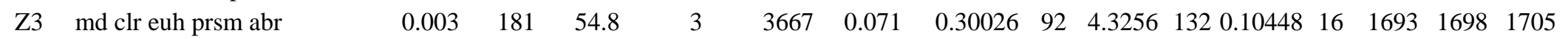

\section{Sheared granodiorite (K00-BR-25; Lat N 38 $33.697^{\prime}$, Long $\mathrm{W}^{105^{\circ}} \mathbf{1 8 . 2 5 8}^{\prime}$ )}

$\begin{array}{llcccccccccccccccc}\text { Z1 } & \text { sm-md pnk sbhd prsm abr } & 0.001 & 125 & 38 & 2 & 1559 & 0.0807 & 0.29885 & 80 & 4.3113 & 132 & 0.10463 & 18 & 1686 & 1696 & 1708 \\ \text { Z2 } & \text { sm-md clr sbhd abr } & 0.002 & 90 & 28.7 & 2 & 2640 & 0.1275 & 0.30225 & 118 & 4.3263 & 172 & 0.10381 & 28 & 1702 & 1698 & 1693 \\ \text { Z3 } & \text { sm-md euh prsm clr abr } & 0.003 & 158 & 49.8 & 13 & 786 & 0.1213 & 0.29929 & 74 & 4.2958 & 122 & 0.10410 & 18 & 1688 & 1693 & 1698 \\ \text { Z4 } & \text { md euh clr abr } & 0.004 & 185 & 58.4 & 10 & 1419 & 0.1307 & 0.2976 & 70 & 4.2775 & 110 & 0.10425 & 16 & 1679 & 1689 & 1701\end{array}$

\section{Cross-cutting pegmatite dike (K00-BR-26; Lat N 38 $33.697^{\prime}$, Long W $105^{\circ} \mathbf{1 8 . 2 5 8}^{\prime}$ )}

$\begin{array}{lllllllllllllllll}\text { Z1 } & \text { v sm clr-pnk sbhd prsm abr } & 0.001 & 210 & 59.1 & 4 & 467 & 0.064 & 0.28056 & 94 & 4.0002 & 268 & 0.10341 & 56 & 1594 & 1634 & 1686 \\ \text { Z2 } & \text { v sm pnk-brn sbhd prsm abr } & 0.001 & 116 & 33 & 6 & 272 & 0.1089 & 0.27299 & 98 & 3.9369 & 496 & 0.10459 & 114 & 1556 & 1621 & 1707 \\ \text { Z3 } & \text { sm pnk euh prsm abr } & 0.002 & 335 & 82.6 & 6 & 2130 & 0.0993 & 0.24061 & 56 & 3.0009 & 76 & 0.09045 & 12 & 1390 & 1408 & 1435\end{array}$

\section{Cross-cutting pegmatite dike (J03-BR4; Lat N 38 $33.697^{\prime}$, Long W $\left.105^{\circ} \mathbf{1 8 . 2 5 8}^{\prime}\right)$}

\begin{tabular}{|c|c|c|c|c|c|c|c|c|c|c|c|c|}
\hline md clr-bge sbhd abr & 0.002 & 480 & 145 & 20 & 1084 & 0.0776 & 0.29664 & 4.333 & $1060.10594 \quad 14$ & 1675 & 1700 & 1731 \\
\hline sm euh clr abr & 0.002 & 92 & 29.2 & 2 & 1469 & 0.1184 & 0.30166 & $76 \quad 4.3307$ & 1360.1041222 & 1700 & 1699 & 1699 \\
\hline md clr sbhe nabr & 0.001 & 119 & 30.3 & 21 & 98 & 0.0937 & 0.25038 & $76 \quad 3.0991$ & 3160.0897780 & 1440 & 1432 & 1421 \\
\hline
\end{tabular}

Abbreviations: abr=abraded; brn=brown; bge=beige; clr=clear; euh=euhedral; lg=large; md=medium; nabr=not abraded; pnk=pink; prsm=prisms; sm=small; sbhd=subhedral; v=very

*Ratios corrected for fractionation, $1 \mathrm{pg}$ and $0.25 \mathrm{pg}$ laboratory $\mathrm{Pb}$ and U blanks respectively and initial common $\mathrm{Pb}$ calculated using $\mathrm{Pb}$ isotopic compositions of Stacey and Kramers (1975). All fractions of zircon are extensively abraded (Krogh, 1982) unless otherwise noted. Two-sigma uncertainties on isotopic ratios are reported after the ratios and refer to the final digits. $\mathrm{Pb}^{\mathrm{R}}$ refers to radiogenic $\mathrm{Pb}$; Common $\mathrm{Pb}^{\mathrm{T}}$ refers to total common $\mathrm{Pb}$. 
Table DR2. U-Pb Isotopic Data and Ages for Detrital Zircon Sample from Phantom Canyon, Colorado

\begin{tabular}{|c|c|c|c|c|c|c|c|c|c|c|c|}
\hline \multirow{3}{*}{ Fraction } & Weight & Concentration & \multicolumn{2}{|c|}{ Measured } & \multicolumn{4}{|c|}{ *Corrected Atomic Ratios } & \multicolumn{3}{|c|}{ Ages (Ma) } \\
\hline & [mg] & $\mathrm{U} \quad \mathrm{Pb}^{\mathrm{R}}$ & Common & ${ }^{206} \mathrm{~Pb}$ & ${ }^{208} \mathrm{~Pb}$ & ${ }^{206} \mathrm{~Pb}$ & ${ }^{207} \mathrm{~Pb}$ & ${ }^{207} \mathrm{~Pb}$ & ${ }^{206} \mathrm{~Pb}$ & ${ }^{207} \mathrm{~Pb}$ & ${ }^{207} \mathrm{~Pb}$ \\
\hline & & [ppm] & $\mathrm{Pb}^{\mathrm{T}}[\mathrm{pg}]$ & ${ }^{204} \mathrm{~Pb}$ & ${ }^{206} \mathrm{~Pb}$ & ${ }^{238} \mathrm{U}$ & ${ }^{235} \mathrm{U}$ & ${ }^{206} \mathrm{~Pb}$ & $\overline{{ }^{238} \mathrm{U}}$ & $\overline{{ }^{235} \mathrm{U}}$ & ${ }^{206} \mathrm{~Pb}$ \\
\hline
\end{tabular}

\section{Phantom Canyon quartzite (J03-PC1; Lat N 38³5.041' ${ }^{\circ}$ Long W 105 $^{\circ} \mathbf{1 8 . 2 5 8}^{\prime}$ )}

$\begin{array}{llccccccccccccccc}\text { Z9 } & \text { sm-md clr pcs abr } & 0.004 & 239 & 52 & 88 & 171 & 0.1421 & 0.2028 & 50 & 2.9159 & 134 & 0.10428 & 36 & 1190 & 1386 & 1702 \\ \text { Z18 } & \text { single md-lg clr abr } & 0.006 & 115 & 35.8 & 7 & 1578 & 0.1919 & 2.7767 & 66 & 3.9914 & 102 & 0.10425 & 16 & 1580 & 1632 & 1701 \\ \text { Z43 } & \text { single md clr abr } & 0.003 & 219 & 38.9 & 3 & 2585 & 0.0799 & 0.17465 & 42 & 2.5093 & 64 & 0.1042 & 14 & 1038 & 1275 & 1700\end{array}$

Abbreviations: abr=abraded; clr=clear; $\mathrm{g}=$ large; $\mathrm{md}=$ =medium; pcs=pieces; sm=small

*Ratios corrected for fractionation, $1 \mathrm{pg}$ and $0.25 \mathrm{pg}$ laboratory $\mathrm{Pb}$ and $\mathrm{U}$ blanks respectively and initial common $\mathrm{Pb}$ calculated using Pb isotopic compositions of Stacey and Kramers (1975). All fractions of zircon are extensively abraded (Krogh, 1982) unless otherwise noted. Two-sigma uncertainties on isotopic ratios are reported after the ratios and refer to the final digits. $\mathrm{Pb}^{\mathrm{R}}$ refers to radiogenic $\mathrm{Pb}$; Common $\mathrm{Pb}{ }^{\mathrm{T}}$ refers to total common Pb. Zircon fraction numbers correspond to LA-ICP-MS analysis numbers reported in Jones (2005). 
Table DR3. Detrital zircon LA-ICP-MS data

\begin{tabular}{|c|c|c|c|c|c|c|c|c|c|c|c|c|c|c|c|}
\hline \multirow[b]{2}{*}{ \# } & \multirow[b]{2}{*}{$\mathrm{N}$} & \multicolumn{7}{|c|}{ Isotopic ratios (corrected) } & \multicolumn{6}{|c|}{ Ages (Ma) } & \multirow[b]{2}{*}{$\begin{array}{c}\text { Disc. } \\
(\%)\end{array}$} \\
\hline & & $\frac{{ }^{206} \mathrm{~Pb}}{{ }^{204} \mathrm{~Pb}^{\dagger}}$ & $\begin{array}{c}{ }^{207} \mathrm{~Pb}^{*} \\
{ }^{235} \mathrm{U} \\
\end{array}$ & $\begin{array}{c} \pm \\
(\mathrm{abs})\end{array}$ & $\begin{array}{l}{ }^{206} \mathrm{~Pb}^{*} \\
{ }^{238} \mathrm{U} \\
\end{array}$ & $\begin{array}{c} \pm \\
(\mathrm{abs})\end{array}$ & $\begin{array}{l}{ }^{207} \mathrm{~Pb}^{*} \\
{ }^{206} \mathrm{~Pb}^{*} \\
\end{array}$ & $\begin{array}{c} \pm \\
(\mathrm{abs})\end{array}$ & $\begin{array}{l}{ }^{207} \mathrm{~Pb}^{*} \\
{ }^{235} \mathrm{U} \\
\end{array}$ & $\begin{array}{c} \pm \\
(\mathrm{abs})\end{array}$ & $\begin{array}{c}{ }^{206} \mathrm{~Pb}^{*} \\
{ }^{238} \mathrm{U} \\
\end{array}$ & $\begin{array}{c} \pm \\
(\mathrm{abs})\end{array}$ & $\begin{array}{l}{ }^{207} \mathrm{~Pb}^{*} \\
{ }^{206} \mathrm{~Pb}^{*}\end{array}$ & $\begin{array}{c} \pm \\
(\mathrm{abs})\end{array}$ & \\
\hline \multicolumn{16}{|c|}{ Sample J03-PC1: Quartzite, Phantom Canyon, Colorado } \\
\hline 1 & 20 & 4,881 & 1.429 & 0.272 & 0.0991 & 0.0182 & 0.1046 & 0.0029 & 901 & 114 & 609 & 107 & 1707 & 50 & -47.92 \\
\hline 2 & 19 & 6,474 & 2.927 & 0.199 & 0.2008 & 0.0119 & 0.1057 & 0.0028 & 1389 & 52 & 1179 & 64 & 1727 & 49 & 15.01 \\
\hline 3 & 19 & 19,041 & 2.386 & 0.359 & 0.1626 & 0.0239 & 0.1064 & 0.0028 & 1238 & 108 & 971 & 132 & 1739 & 48 & -27.49 \\
\hline 6 & 14 & 5,210 & 2.125 & 0.148 & 0.1467 & 0.0091 & 0.1050 & 0.0030 & 1157 & 48 & 883 & 51 & 1715 & 52 & -31.08 \\
\hline 7 & 9 & 9,990 & 1.682 & 0.126 & 0.1167 & 0.0080 & 0.1045 & 0.0031 & 1002 & 48 & 711 & 46 & 1706 & 54 & -40.81 \\
\hline 8 & 18 & 17,429 & 3.840 & 0.235 & 0.2626 & 0.0136 & 0.1060 & 0.0028 & 1601 & 49 & 1503 & 69 & 1732 & 48 & 3.82 \\
\hline 9 & 10 & 8,340 & 3.054 & 0.462 & 0.2167 & 0.0315 & 0.1022 & 0.0029 & 1421 & 116 & 1265 & 167 & 1665 & 53 & 9.37 \\
\hline 14 & 19 & 16,990 & 3.740 & 0.227 & 0.2559 & 0.0131 & 0.1060 & 0.0028 & 1580 & 49 & 1469 & 67 & 1732 & 49 & 4.62 \\
\hline 15 & 19 & 5,613 & 1.602 & 0.157 & 0.1122 & 0.0104 & 0.1035 & 0.0028 & 971 & 61 & 686 & 60 & 1688 & 50 & -41.59 \\
\hline 16 & 12 & 13,224 & 3.648 & 0.264 & 0.2520 & 0.0160 & 0.1050 & 0.0029 & 1560 & 58 & 1449 & 83 & 1714 & 51 & 4.78 \\
\hline 17 & 11 & 13,916 & 4.103 & 0.317 & 0.2806 & 0.0191 & 0.1061 & 0.0030 & 1655 & 63 & 1594 & 96 & 1733 & 52 & 2.03 \\
\hline 18 & 19 & 22,161 & 3.827 & 0.241 & 0.2655 & 0.0143 & 0.1045 & 0.0027 & 1598 & 51 & 1518 & 73 & 1706 & 48 & 3.05 \\
\hline 19 & 20 & 5,661 & 3.907 & 0.293 & 0.2593 & 0.0172 & 0.1093 & 0.0029 & 1615 & 61 & 1486 & 88 & 1787 & 48 & 5.25 \\
\hline 20 & 20 & 8,443 & 3.708 & 0.253 & 0.2582 & 0.0152 & 0.1042 & 0.0028 & 1573 & 54 & 1480 & 78 & 1700 & 49 & 3.75 \\
\hline 21 & 19 & 2,432 & 1.248 & 0.079 & 0.0890 & 0.0048 & 0.1017 & 0.0028 & 822 & 36 & 549 & 29 & 1656 & 51 & -49.68 \\
\hline 22 & 19 & 6,910 & 2.652 & 0.308 & 0.1832 & 0.0201 & 0.1050 & 0.0028 & 1315 & 86 & 1084 & 110 & 1714 & 49 & 20.11 \\
\hline 30 & 18 & 3,688 & 1.864 & 0.155 & 0.1286 & 0.0097 & 0.1051 & 0.0029 & 1068 & 55 & 780 & 55 & 1716 & 51 & -36.99 \\
\hline 31 & 20 & 7,794 & 1.451 & 0.232 & 0.1000 & 0.0155 & 0.1053 & 0.0028 & 910 & 96 & 614 & 91 & 1719 & 49 & -48.19 \\
\hline 32 & 11 & 11,469 & 4.078 & 0.329 & 0.2723 & 0.0196 & 0.1086 & 0.0031 & 1650 & 66 & 1552 & 99 & 1776 & 51 & 3.53 \\
\hline 33 & 12 & 1,562 & 3.616 & 0.291 & 0.2416 & 0.0149 & 0.1086 & 0.0041 & 1553 & 64 & 1395 & 77 & 1775 & 69 & 7.58 \\
\hline 34 & 19 & 8,816 & 2.649 & 0.284 & 0.1778 & 0.0180 & 0.1080 & 0.0030 & 1314 & 79 & 1055 & 99 & 1766 & 51 & 24.32 \\
\hline 35 & 17 & 11,807 & 3.478 & 0.289 & 0.2359 & 0.0179 & 0.1069 & 0.0029 & 1522 & 65 & 1365 & 93 & 1748 & 49 & 7.88 \\
\hline 36 & 20 & 17,169 & 2.993 & 0.194 & 0.2052 & 0.0117 & 0.1058 & 0.0028 & 1406 & 49 & 1203 & 62 & 1728 & 48 & 13.85 \\
\hline 37 & 17 & 9,855 & 3.225 & 0.195 & 0.2222 & 0.0113 & 0.1053 & 0.0029 & 1463 & 47 & 1293 & 60 & 1719 & 50 & 9.72 \\
\hline 38 & 19 & 12,041 & 3.395 & 0.217 & 0.2343 & 0.0129 & 0.1051 & 0.0028 & 1503 & 50 & 1357 & 67 & 1716 & 49 & 7.42 \\
\hline 39 & 9 & 3,704 & 1.744 & 0.164 & 0.1278 & 0.0108 & 0.0990 & 0.0033 & 1025 & 61 & 775 & 62 & 1605 & 62 & -32.21 \\
\hline 40 & 19 & 11,137 & 3.446 & 0.210 & 0.2350 & 0.0120 & 0.1064 & 0.0029 & 1515 & 48 & 1361 & 63 & 1738 & 50 & 7.81 \\
\hline 41 & 19 & 8,924 & 3.545 & 0.220 & 0.2445 & 0.0128 & 0.1052 & 0.0028 & 1537 & 49 & 1410 & 66 & 1717 & 50 & 5.85 \\
\hline 42 & 15 & 6,592 & 2.962 & 0.196 & 0.2022 & 0.0115 & 0.1063 & 0.0029 & 1398 & 50 & 1187 & 62 & 1736 & 50 & 14.93 \\
\hline 43 & 20 & 11,174 & 2.465 & 0.154 & 0.1701 & 0.0094 & 0.1051 & 0.0028 & 1262 & 45 & 1013 & 52 & 1716 & 49 & 25.41 \\
\hline 44 & 19 & 18,860 & 4.172 & 0.285 & 0.2869 & 0.0171 & 0.1055 & 0.0028 & 1669 & 56 & 1626 & 86 & 1723 & 49 & 1.35 \\
\hline 45 & 19 & 18,549 & 3.299 & 0.213 & 0.2255 & 0.0127 & 0.1061 & 0.0028 & 1481 & 50 & 1311 & 67 & 1734 & 49 & 9.44 \\
\hline
\end{tabular}


Isotopic ratios (corrected)

Ages (Ma)

\begin{tabular}{|c|c|c|c|c|c|c|c|c|c|c|c|c|c|c|c|}
\hline$\#$ & $\mathrm{~N}$ & $\frac{{ }^{206} \mathrm{~Pb}}{{ }^{204} \mathrm{~Pb}^{\dagger}}$ & $\begin{array}{l}{ }^{207} \mathrm{~Pb}^{*} \\
{ }^{235} \mathrm{U}\end{array}$ & $\begin{array}{c} \pm \\
\text { (abs) }\end{array}$ & $\begin{array}{l}{ }^{206} \mathrm{~Pb}^{*} \\
{ }^{238} \mathrm{U}\end{array}$ & $\begin{array}{c} \pm \\
(\mathrm{abs})\end{array}$ & $\begin{array}{l}{ }^{207} \mathrm{~Pb}^{*} \\
{ }^{206} \mathrm{~Pb}^{*}\end{array}$ & $\begin{array}{c} \pm \\
\text { (abs) }\end{array}$ & $\begin{array}{c}{ }^{207} \mathrm{~Pb}^{*} \\
{ }^{235} \mathrm{U}\end{array}$ & $\begin{array}{c} \pm \\
\text { (abs) }\end{array}$ & $\begin{array}{c}{ }^{206} \mathrm{~Pb}^{*} \\
{ }^{238} \mathrm{U}\end{array}$ & $\begin{array}{c} \pm \\
(\mathrm{abs})\end{array}$ & $\begin{array}{l}{ }^{207} \mathrm{~Pb}^{*} \\
{ }^{206} \mathrm{~Pb}^{*}\end{array}$ & $\begin{array}{c} \pm \\
(\mathrm{abs})\end{array}$ & $\begin{array}{l}\text { Disc. } \\
(\%)\end{array}$ \\
\hline 46 & 14 & 7,733 & 3.455 & 0.215 & 0.2360 & 0.0123 & 0.1062 & 0.0030 & 1517 & 49 & 1366 & 64 & 1734 & 51 & 7.56 \\
\hline 47 & 16 & 6,672 & 3.431 & 1.042 & 0.2368 & 0.0701 & 0.1051 & 0.0029 & 1512 & 239 & 1370 & 365 & 1716 & 50 & 7.01 \\
\hline 48 & 19 & 18,424 & 3.776 & 0.230 & 0.2582 & 0.0133 & 0.1060 & 0.0028 & 1588 & 49 & 1481 & 68 & 1733 & 49 & 4.35 \\
\hline 49 & 13 & 5,704 & 1.678 & 0.207 & 0.1155 & 0.0136 & 0.1053 & 0.0030 & 1000 & 78 & 705 & 78 & 1720 & 52 & -41.91 \\
\hline 50 & 19 & 40,608 & 3.477 & 0.206 & 0.2369 & 0.0119 & 0.1065 & 0.0028 & 1522 & 47 & 1370 & 62 & 1739 & 48 & 7.54 \\
\hline 51 & 19 & 29,102 & 3.071 & 0.270 & 0.2093 & 0.0172 & 0.1064 & 0.0028 & 1426 & 67 & 1225 & 92 & 1739 & 48 & 13.15 \\
\hline 52 & 20 & 24,861 & 3.770 & 0.229 & 0.2550 & 0.0130 & 0.1072 & 0.0029 & 1586 & 49 & 1464 & 67 & 1752 & 49 & 5.13 \\
\hline 53 & 15 & 9,928 & 3.101 & 0.262 & 0.2146 & 0.0166 & 0.1048 & 0.0028 & 1433 & 65 & 1253 & 88 & 1711 & 50 & 11.08 \\
\hline 54 & 19 & 26,882 & 3.738 & 0.225 & 0.2552 & 0.0130 & 0.1062 & 0.0028 & 1579 & 48 & 1465 & 67 & 1736 & 48 & 4.78 \\
\hline 55 & 17 & 10,922 & 4.075 & 0.266 & 0.2787 & 0.0155 & 0.1060 & 0.0028 & 1649 & 53 & 1585 & 78 & 1732 & 49 & 2.18 \\
\hline 56 & 19 & 15,508 & 4.051 & 0.300 & 0.2765 & 0.0183 & 0.1063 & 0.0028 & 1644 & 60 & 1573 & 92 & 1736 & 48 & 2.46 \\
\hline 57 & 20 & 4,183 & 2.031 & 0.116 & 0.1399 & 0.0068 & 0.1053 & 0.0028 & 1126 & 39 & 844 & 38 & 1719 & 50 & -33.38 \\
\hline 58 & 16 & 13,240 & 3.558 & 0.228 & 0.2429 & 0.0133 & 0.1062 & 0.0029 & 1540 & 51 & 1402 & 69 & 1736 & 50 & 6.49 \\
\hline 59 & 20 & 15,790 & 4.259 & 0.299 & 0.2912 & 0.0179 & 0.1061 & 0.0028 & 1685 & 58 & 1647 & 90 & 1733 & 49 & 1.16 \\
\hline 60 & 19 & 8,072 & 2.607 & 0.150 & 0.1779 & 0.0087 & 0.1063 & 0.0028 & 1303 & 42 & 1055 & 48 & 1737 & 49 & 23.04 \\
\hline 61 & 20 & 9,552 & 3.475 & 0.217 & 0.2205 & 0.0118 & 0.1143 & 0.0030 & 1522 & 49 & 1285 & 62 & 1869 & 48 & 14.26 \\
\hline 62 & 17 & 4,184 & 2.127 & 0.123 & 0.1462 & 0.0071 & 0.1055 & 0.0029 & 1158 & 40 & 880 & 40 & 1723 & 50 & -31.56 \\
\hline 63 & 19 & 7,407 & 3.544 & 0.217 & 0.2448 & 0.0125 & 0.1050 & 0.0029 & 1537 & 48 & 1412 & 65 & 1714 & 50 & 5.75 \\
\hline 64 & 9 & 24,965 & 3.421 & 0.228 & 0.2401 & 0.0135 & 0.1034 & 0.0030 & 1509 & 52 & 1387 & 70 & 1685 & 54 & 5.82 \\
\hline 65 & 17 & 6,037 & 1.447 & 0.097 & 0.0996 & 0.0060 & 0.1054 & 0.0029 & 909 & 40 & 612 & 35 & 1721 & 50 & -48.48 \\
\hline 66 & 18 & 10,188 & 3.982 & 0.274 & 0.2737 & 0.0163 & 0.1055 & 0.0029 & 1631 & 56 & 1560 & 82 & 1724 & 50 & 2.51 \\
\hline 67 & 19 & 23,875 & 3.258 & 0.215 & 0.2222 & 0.0129 & 0.1063 & 0.0028 & 1471 & 51 & 1293 & 68 & 1738 & 48 & 10.20 \\
\hline 68 & 19 & 17,564 & 3.766 & 0.230 & 0.2566 & 0.0132 & 0.1064 & 0.0028 & 1586 & 49 & 1473 & 68 & 1739 & 49 & 4.67 \\
\hline 69 & 19 & 13,473 & 3.569 & 0.215 & 0.2436 & 0.0123 & 0.1063 & 0.0028 & 1543 & 48 & 1406 & 64 & 1736 & 49 & 6.39 \\
\hline 70 & 16 & 17,318 & 3.991 & 0.257 & 0.2799 & 0.0153 & 0.1034 & 0.0028 & 1632 & 52 & 1591 & 77 & 1686 & 50 & 1.38 \\
\hline 71 & 19 & 11,463 & 3.823 & 0.234 & 0.2584 & 0.0132 & 0.1073 & 0.0029 & 1598 & 49 & 1482 & 68 & 1754 & 49 & 4.73 \\
\hline 72 & 19 & 12,107 & 3.336 & 0.200 & 0.2276 & 0.0115 & 0.1063 & 0.0028 & 1489 & 47 & 1322 & 61 & 1736 & 49 & 9.10 \\
\hline 73 & 11 & 7,017 & 1.938 & 0.171 & 0.1330 & 0.0109 & 0.1057 & 0.0030 & 1094 & 59 & 805 & 62 & 1726 & 52 & -35.92 \\
\hline 74 & 17 & 4,304 & 2.081 & 0.133 & 0.1430 & 0.0079 & 0.1056 & 0.0029 & 1143 & 44 & 861 & 45 & 1725 & 50 & -32.65 \\
\hline 75 & 18 & 18,381 & 3.329 & 0.220 & 0.2266 & 0.0131 & 0.1065 & 0.0028 & 1488 & 52 & 1317 & 69 & 1741 & 48 & 9.41 \\
\hline 76 & 14 & 15,975 & 3.538 & 0.226 & 0.2477 & 0.0135 & 0.1036 & 0.0028 & 1536 & 51 & 1426 & 70 & 1689 & 50 & 4.85 \\
\hline 77 & 19 & 22,511 & 4.337 & 0.276 & 0.2947 & 0.0160 & 0.1067 & 0.0028 & 1700 & 53 & 1665 & 80 & 1744 & 48 & 1.05 \\
\hline 78 & 19 & 18,906 & 3.740 & 0.232 & 0.2553 & 0.0135 & 0.1063 & 0.0028 & 1580 & 50 & 1466 & 69 & 1736 & 48 & 4.78 \\
\hline 79 & 13 & 14,875 & 3.741 & 0.249 & 0.2519 & 0.0143 & 0.1077 & 0.0030 & 1580 & 53 & 1448 & 74 & 1761 & 51 & 5.73 \\
\hline 80 & 20 & 4,459 & 3.672 & 0.235 & 0.2554 & 0.0137 & 0.1043 & 0.0028 & 1565 & 51 & 1466 & 70 & 1702 & 50 & 4.11 \\
\hline 81 & 20 & 20,412 & 5.950 & 0.440 & 0.4064 & 0.0260 & 0.1062 & 0.0028 & 1969 & 64 & 2199 & 119 & 1735 & 48 & -2.95 \\
\hline 82 & 15 & 9,668 & 4.308 & 0.285 & 0.2953 & 0.0165 & 0.1058 & 0.0029 & 1695 & 54 & 1668 & 82 & 1729 & 50 & 0.80 \\
\hline 83 & 19 & 9,783 & 4.359 & 0.304 & 0.3017 & 0.0183 & 0.1048 & 0.0028 & 1705 & 58 & 1700 & 90 & 1711 & 49 & 0.14 \\
\hline 84 & 16 & 1,524 & 2.081 & 0.146 & 0.1509 & 0.0087 & 0.1000 & 0.0029 & 1142 & 48 & 906 & 49 & 1624 & 54 & -26.12 \\
\hline 85 & 18 & 11,449 & 4.445 & 0.307 & 0.3044 & 0.0182 & 0.1059 & 0.0028 & 1721 & 57 & 1713 & 90 & 1730 & 49 & 0.21 \\
\hline 86 & 11 & 22,354 & 5.525 & 0.790 & 0.3787 & 0.0520 & 0.1058 & 0.0030 & 1905 & 123 & 2070 & 243 & 1729 & 52 & -2.57 \\
\hline 87 & 17 & 3,540 & 2.513 & 0.166 & 0.1751 & 0.0099 & 0.1041 & 0.0029 & 1276 & 48 & 1040 & 55 & 1699 & 51 & 22.54 \\
\hline 88 & 19 & 9,417 & 4.200 & 0.271 & 0.2866 & 0.0157 & 0.1063 & 0.0028 & 1674 & 53 & 1625 & 78 & 1736 & 49 & 1.56 \\
\hline 89 & 19 & 5,235 & 4.409 & 0.297 & 0.3002 & 0.0170 & 0.1065 & 0.0029 & 1714 & 56 & 1692 & 84 & 1741 & 50 & 0.62 \\
\hline 90 & 20 & 8,744 & 4.066 & 0.278 & 0.2789 & 0.0165 & 0.1057 & 0.0028 & 1648 & 56 & 1586 & 83 & 1727 & 49 & 2.08 \\
\hline 91 & 19 & 11,111 & 3.875 & 0.278 & 0.2628 & 0.0166 & 0.1069 & 0.0028 & 1609 & 58 & 1504 & 85 & 1748 & 48 & 4.07 \\
\hline
\end{tabular}


Isotopic ratios (corrected)

Ages (Ma)

\begin{tabular}{|c|c|c|c|c|c|c|c|c|c|c|c|c|c|c|c|}
\hline \# & $\mathrm{N}$ & $\frac{{ }^{206} \mathrm{~Pb}}{{ }^{204} \mathrm{~Pb}^{\dagger}}$ & $\begin{array}{c}{ }^{207} \mathrm{~Pb}^{*} \\
{ }^{235} \mathrm{U} \\
\end{array}$ & $\begin{array}{c} \pm \\
(\mathrm{abs}) \\
\end{array}$ & $\begin{array}{c}{ }^{206} \mathrm{~Pb}^{*} \\
{ }^{238} \mathrm{U} \\
\end{array}$ & $\begin{array}{c} \pm \\
\text { (abs) }\end{array}$ & $\begin{array}{l}{ }^{207} \mathrm{~Pb}^{*} \\
{ }^{206} \mathrm{~Pb}^{*}\end{array}$ & $\begin{array}{c} \pm \\
\text { (abs) }\end{array}$ & $\begin{array}{c}{ }^{207} \mathrm{~Pb}^{*} \\
{ }^{235} \mathrm{U} \\
\end{array}$ & $\begin{array}{c} \pm \\
\text { (abs) }\end{array}$ & $\begin{array}{c}{ }^{206} \mathrm{~Pb}^{*} \\
{ }^{238} \mathrm{U} \\
\end{array}$ & $\begin{array}{c} \pm \\
\text { (abs) }\end{array}$ & $\begin{array}{l}{ }^{207} \mathrm{~Pb}^{*} \\
{ }^{206} \mathrm{~Pb}^{*}\end{array}$ & $\begin{array}{c} \pm \\
(\mathrm{abs})\end{array}$ & $\begin{array}{l}\text { Disc. } \\
(\%)\end{array}$ \\
\hline 92 & 18 & 14,795 & 4.062 & 0.253 & 0.2672 & 0.0140 & 0.1103 & 0.0030 & 1647 & 51 & 1526 & 71 & 1804 & 49 & 4.59 \\
\hline 93 & 20 & 5,643 & 4.693 & 0.458 & 0.3284 & 0.0292 & 0.1036 & 0.0028 & 1766 & 82 & 1831 & 142 & 1690 & 51 & -1.45 \\
\hline 94 & 19 & 8,925 & 4.305 & 0.281 & 0.2985 & 0.0165 & 0.1046 & 0.0028 & 1694 & 54 & 1684 & 82 & 1707 & 49 & 0.30 \\
\hline 95 & 15 & 5,882 & 2.859 & 0.352 & 0.1963 & 0.0228 & 0.1056 & 0.0029 & 1371 & 93 & 1155 & 123 & 1725 & 50 & 16.26 \\
\hline 97 & 16 & 2,822 & 2.893 & 0.192 & 0.1977 & 0.0110 & 0.1061 & 0.0030 & 1380 & 50 & 1163 & 59 & 1734 & 51 & 16.13 \\
\hline 98 & 19 & 22,603 & 4.089 & 0.256 & 0.2796 & 0.0149 & 0.1061 & 0.0028 & 1652 & 51 & 1589 & 75 & 1733 & 48 & 2.12 \\
\hline 99 & 18 & 18,793 & 3.606 & 0.221 & 0.2453 & 0.0128 & 0.1066 & 0.0028 & 1551 & 49 & 1414 & 66 & 1742 & 49 & 6.27 \\
\hline 100 & 20 & 14,023 & 3.472 & 0.208 & 0.2388 & 0.0120 & 0.1055 & 0.0028 & 1521 & 47 & 1380 & 63 & 1722 & 48 & 6.84 \\
\hline 101 & 18 & 22,272 & 3.615 & 0.226 & 0.2529 & 0.0135 & 0.1037 & 0.0027 & 1553 & 50 & 1454 & 69 & 1691 & 49 & 4.20 \\
\hline 106 & 19 & 6,469 & 2.843 & 0.525 & 0.1947 & 0.0348 & 0.1059 & 0.0028 & 1367 & 139 & 1147 & 188 & 1730 & 49 & 16.89 \\
\hline 107 & 20 & 7,707 & 4.013 & 0.256 & 0.2752 & 0.0149 & 0.1057 & 0.0028 & 1637 & 52 & 1567 & 75 & 1727 & 49 & 2.43 \\
\hline 108 & 19 & 15,033 & 5.507 & 0.402 & 0.3722 & 0.0236 & 0.1073 & 0.0028 & 1902 & 63 & 2040 & 111 & 1754 & 48 & -2.26 \\
\hline 109 & 19 & 19,519 & 3.987 & 0.261 & 0.2723 & 0.0154 & 0.1062 & 0.0028 & 1632 & 53 & 1553 & 78 & 1735 & 48 & 2.83 \\
\hline
\end{tabular}

\begin{tabular}{|c|c|c|c|c|c|c|c|c|c|c|c|c|c|c|c|}
\hline \multicolumn{16}{|c|}{ Sample J01-BR1: Quartzite, Blue Ridge, Colorado } \\
\hline 1 & 20 & 15,227 & 4.628 & 0.244 & 0.3076 & 0.0137 & 0.1091 & 0.0029 & 1754 & 44 & 1729 & 68 & 1785 & 48 & 0.68 \\
\hline 2 & 19 & 12,684 & 4.308 & 0.227 & 0.2917 & 0.0129 & 0.1071 & 0.0029 & 1695 & 43 & 1650 & 64 & 1751 & 49 & 1.37 \\
\hline 4 & 17 & 16,626 & 4.736 & 0.251 & 0.3054 & 0.0136 & 0.1125 & 0.0030 & 1774 & 44 & 1718 & 67 & 1840 & 48 & 1.55 \\
\hline 5 & 15 & 1,577 & 3.681 & 0.282 & 0.2321 & 0.0152 & 0.1150 & 0.0035 & 1567 & 61 & 1345 & 80 & 1880 & 54 & 11.92 \\
\hline 6 & 19 & 15,473 & 6.446 & 0.394 & 0.4429 & 0.0232 & 0.1055 & 0.0028 & 2039 & 54 & 2364 & 103 & 1724 & 49 & -3.27 \\
\hline 8 & 8 & 1,845 & 6.406 & 1.569 & 0.4088 & 0.0930 & 0.1137 & 0.0036 & 2033 & 215 & 2209 & 426 & 1859 & 58 & -2.29 \\
\hline 9 & 20 & 12,230 & 5.245 & 0.283 & 0.3326 & 0.0150 & 0.1144 & 0.0031 & 1860 & 46 & 1851 & 73 & 1870 & 48 & 0.20 \\
\hline 10 & 17 & 17,232 & 4.411 & 0.235 & 0.3011 & 0.0136 & 0.1062 & 0.0028 & 1714 & 44 & 1697 & 67 & 1736 & 49 & 0.49 \\
\hline 11 & 18 & 10,740 & 4.991 & 0.268 & 0.3165 & 0.0143 & 0.1144 & 0.0031 & 1818 & 45 & 1772 & 70 & 1870 & 48 & 1.16 \\
\hline 12 & 13 & 3,508 & 6.117 & 0.422 & 0.4196 & 0.0245 & 0.1057 & 0.0031 & 1993 & 60 & 2258 & 111 & 1727 & 53 & -3.12 \\
\hline 16 & 11 & 1,614 & 2.767 & 0.404 & 0.1865 & 0.0248 & 0.1076 & 0.0033 & 1347 & 109 & 1102 & 135 & 1759 & 56 & 20.70 \\
\hline 17 & 19 & 6,800 & 4.407 & 0.236 & 0.3003 & 0.0134 & 0.1064 & 0.0029 & 1714 & 44 & 1693 & 66 & 1739 & 49 & 0.59 \\
\hline 18 & 19 & 13,283 & 4.564 & 0.242 & 0.3056 & 0.0137 & 0.1083 & 0.0029 & 1743 & 44 & 1719 & 67 & 1771 & 48 & 0.64 \\
\hline 19 & 15 & 2,533 & 4.657 & 0.328 & 0.3165 & 0.0192 & 0.1067 & 0.0030 & 1760 & 59 & 1773 & 94 & 1744 & 52 & -0.32 \\
\hline 20 & 20 & 14,435 & 4.079 & 0.211 & 0.2724 & 0.0119 & 0.1086 & 0.0028 & 1650 & 42 & 1553 & 60 & 1776 & 48 & 3.51 \\
\hline 21 & 19 & 6,328 & 4.219 & 0.223 & 0.2813 & 0.0123 & 0.1088 & 0.0029 & 1678 & 43 & 1598 & 62 & 1779 & 49 & 2.67 \\
\hline 22 & 19 & 17,772 & 4.618 & 0.244 & 0.3093 & 0.0138 & 0.1083 & 0.0028 & 1753 & 44 & 1737 & 68 & 1771 & 48 & 0.41 \\
\hline 23 & 20 & 12,293 & 4.681 & 0.248 & 0.3088 & 0.0138 & 0.1099 & 0.0029 & 1764 & 44 & 1735 & 68 & 1798 & 48 & 0.77 \\
\hline 24 & 19 & 8,526 & 4.363 & 0.231 & 0.3001 & 0.0133 & 0.1054 & 0.0028 & 1705 & 44 & 1692 & 66 & 1722 & 49 & 0.38 \\
\hline 25 & 20 & 9,336 & 4.431 & 0.234 & 0.3042 & 0.0134 & 0.1057 & 0.0028 & 1718 & 44 & 1712 & 66 & 1726 & 49 & 0.17 \\
\hline
\end{tabular}


Isotopic ratios (corrected)

Ages (Ma)

\begin{tabular}{|c|c|c|c|c|c|c|c|c|c|c|c|c|c|c|c|}
\hline$\#$ & $\mathrm{~N}$ & $\frac{{ }^{206} \mathrm{~Pb}}{{ }^{204} \mathrm{~Pb}^{\dagger}}$ & $\begin{array}{c}{ }^{207} \mathrm{~Pb}^{*} \\
{ }^{235} \mathrm{U}\end{array}$ & $\begin{array}{c} \pm \\
\text { (abs) }\end{array}$ & $\begin{array}{l}{ }^{206} \mathrm{~Pb}^{*} \\
{ }^{238} \mathrm{U}\end{array}$ & $\begin{array}{c} \pm \\
(\mathrm{abs})\end{array}$ & $\begin{array}{l}{ }^{207} \mathrm{~Pb}^{*} \\
{ }^{206} \mathrm{~Pb}^{*}\end{array}$ & $\begin{array}{c} \pm \\
\text { (abs) }\end{array}$ & $\begin{array}{c}{ }^{207} \mathrm{~Pb}^{*} \\
{ }^{235} \mathrm{U}\end{array}$ & $\begin{array}{c} \pm \\
\text { (abs) }\end{array}$ & $\begin{array}{c}{ }^{206} \mathrm{~Pb}^{*} \\
{ }^{238} \mathrm{U}\end{array}$ & $\begin{array}{c} \pm \\
(\mathrm{abs})\end{array}$ & $\begin{array}{l}{ }^{207} \mathrm{~Pb}^{*} \\
{ }^{206} \mathrm{~Pb}^{*}\end{array}$ & $\begin{array}{c} \pm \\
(\mathrm{abs})\end{array}$ & $\begin{array}{l}\text { Disc. } \\
(\%)\end{array}$ \\
\hline 27 & 12 & 6,289 & 4.344 & 0.259 & 0.3007 & 0.0152 & 0.1048 & 0.0030 & 1702 & 49 & 1695 & 75 & 1711 & 52 & 0.20 \\
\hline 28 & 19 & 7,771 & 4.877 & 0.277 & 0.3383 & 0.0162 & 0.1046 & 0.0029 & 1798 & 48 & 1878 & 78 & 1706 & 51 & -1.67 \\
\hline 29 & 15 & 14,535 & 4.599 & 0.256 & 0.3182 & 0.0150 & 0.1048 & 0.0029 & 1749 & 46 & 1781 & 73 & 1711 & 50 & -0.78 \\
\hline 30 & 17 & 17,097 & 4.576 & 0.247 & 0.3112 & 0.0142 & 0.1066 & 0.0029 & 1745 & 45 & 1747 & 70 & 1743 & 49 & -0.05 \\
\hline 31 & 14 & 7,226 & 5.824 & 0.355 & 0.4008 & 0.0206 & 0.1054 & 0.0029 & 1950 & 53 & 2173 & 95 & 1721 & 50 & -2.96 \\
\hline 32 & 19 & 14,747 & 4.468 & 0.235 & 0.2972 & 0.0131 & 0.1090 & 0.0029 & 1725 & 44 & 1677 & 65 & 1784 & 49 & 1.40 \\
\hline 33 & 20 & 28,325 & 5.063 & 0.269 & 0.3277 & 0.0147 & 0.1121 & 0.0029 & 1830 & 45 & 1827 & 72 & 1833 & 47 & 0.06 \\
\hline 34 & 12 & 1,056 & 4.461 & 0.387 & 0.2686 & 0.0196 & 0.1204 & 0.0037 & 1724 & 72 & 1534 & 100 & 1963 & 55 & 7.42 \\
\hline 35 & 19 & 2,148 & 6.309 & 0.412 & 0.4305 & 0.0230 & 0.1063 & 0.0029 & 2020 & 57 & 2308 & 104 & 1737 & 51 & -3.15 \\
\hline 36 & 19 & 7,471 & 4.144 & 0.219 & 0.2870 & 0.0126 & 0.1047 & 0.0028 & 1663 & 43 & 1626 & 63 & 1709 & 50 & 1.15 \\
\hline 37 & 20 & 15,844 & 4.634 & 0.250 & 0.3178 & 0.0145 & 0.1058 & 0.0028 & 1755 & 45 & 1779 & 71 & 1728 & 49 & -0.58 \\
\hline 38 & 13 & 2,376 & 5.879 & 0.403 & 0.3723 & 0.0213 & 0.1145 & 0.0034 & 1958 & 60 & 2040 & 100 & 1872 & 54 & -1.38 \\
\hline 39 & 20 & 14,135 & 6.533 & 0.423 & 0.4481 & 0.0252 & 0.1057 & 0.0028 & 2050 & 57 & 2387 & 112 & 1727 & 49 & -3.27 \\
\hline 40 & 15 & 8,385 & 4.553 & 0.247 & 0.3058 & 0.0139 & 0.1080 & 0.0029 & 1741 & 45 & 1720 & 69 & 1765 & 50 & 0.56 \\
\hline 41 & 16 & 6,477 & 5.425 & 0.299 & 0.3281 & 0.0151 & 0.1199 & 0.0032 & 1889 & 47 & 1829 & 73 & 1955 & 48 & 1.43 \\
\hline 42 & 17 & 10,030 & 5.007 & 0.312 & 0.3427 & 0.0186 & 0.1060 & 0.0028 & 1820 & 53 & 1899 & 89 & 1731 & 49 & -1.60 \\
\hline 43 & 18 & 9,259 & 5.176 & 0.353 & 0.3570 & 0.0217 & 0.1052 & 0.0028 & 1849 & 58 & 1968 & 103 & 1717 & 49 & -2.17 \\
\hline 44 & 19 & 11,160 & 4.362 & 0.229 & 0.2992 & 0.0132 & 0.1057 & 0.0028 & 1705 & 43 & 1687 & 66 & 1727 & 49 & 0.50 \\
\hline 45 & 16 & 7,793 & 4.948 & 0.305 & 0.3413 & 0.0182 & 0.1052 & 0.0028 & 1811 & 52 & 1893 & 88 & 1717 & 50 & -1.68 \\
\hline 46 & 19 & 6,473 & 3.800 & 0.199 & 0.2652 & 0.0115 & 0.1039 & 0.0028 & 1593 & 42 & 1516 & 59 & 1696 & 50 & 2.90 \\
\hline 47 & 10 & 1,117 & 5.748 & 0.562 & 0.3937 & 0.0320 & 0.1059 & 0.0034 & 1939 & 85 & 2140 & 148 & 1730 & 59 & -2.82 \\
\hline 48 & 13 & 7,113 & 3.856 & 0.210 & 0.2629 & 0.0121 & 0.1064 & 0.0030 & 1604 & 44 & 1505 & 62 & 1738 & 51 & 3.89 \\
\hline 49 & 18 & 7,093 & 4.026 & 0.224 & 0.2753 & 0.0129 & 0.1061 & 0.0029 & 1640 & 45 & 1568 & 65 & 1733 & 50 & 2.50 \\
\hline 50 & 20 & 6,532 & 4.142 & 0.222 & 0.2854 & 0.0128 & 0.1052 & 0.0028 & 1663 & 44 & 1619 & 64 & 1719 & 49 & 1.40 \\
\hline 51 & 19 & 6,723 & 4.612 & 0.248 & 0.3077 & 0.0137 & 0.1087 & 0.0029 & 1751 & 45 & 1729 & 68 & 1778 & 49 & 0.60 \\
\hline 52 & 13 & 8,445 & 5.579 & 0.340 & 0.3835 & 0.0198 & 0.1055 & 0.0029 & 1913 & 52 & 2093 & 92 & 1723 & 51 & -2.70 \\
\hline 53 & 19 & 22,557 & 5.252 & 0.278 & 0.3200 & 0.0144 & 0.1190 & 0.0031 & 1861 & 45 & 1790 & 70 & 1942 & 47 & 1.81 \\
\hline 54 & 11 & 948 & 1.862 & 0.268 & 0.1260 & 0.0157 & 0.1072 & 0.0035 & 1068 & 95 & 765 & 90 & 1752 & 60 & -39.54 \\
\hline 55 & 16 & 8,716 & 4.158 & 0.227 & 0.2857 & 0.0132 & 0.1055 & 0.0029 & 1666 & 45 & 1620 & 66 & 1724 & 50 & 1.45 \\
\hline 56 & 20 & 9,568 & 4.682 & 0.262 & 0.3233 & 0.0154 & 0.1050 & 0.0028 & 1764 & 47 & 1806 & 75 & 1715 & 49 & -0.98 \\
\hline 57 & 14 & 5,834 & 4.654 & 0.297 & 0.3223 & 0.0178 & 0.1047 & 0.0029 & 1759 & 53 & 1801 & 87 & 1710 & 52 & -0.99 \\
\hline 58 & 17 & 7,431 & 4.418 & 0.241 & 0.3073 & 0.0140 & 0.1043 & 0.0028 & 1716 & 45 & 1728 & 69 & 1701 & 50 & -0.31 \\
\hline 59 & 19 & 10,899 & 4.958 & 0.301 & 0.3415 & 0.0181 & 0.1053 & 0.0028 & 1812 & 51 & 1894 & 87 & 1720 & 49 & -1.66 \\
\hline 60 & 19 & 11,170 & 5.882 & 0.363 & 0.4048 & 0.0216 & 0.1054 & 0.0028 & 1959 & 54 & 2191 & 99 & 1721 & 49 & -3.01 \\
\hline 61 & 17 & 12,223 & 4.587 & 0.247 & 0.3043 & 0.0138 & 0.1093 & 0.0029 & 1747 & 45 & 1713 & 68 & 1788 & 49 & 0.95 \\
\hline 62 & 20 & 8,084 & 15.172 & 1.142 & 0.5688 & 0.0373 & 0.1934 & 0.0051 & 2826 & 72 & 2903 & 153 & 2772 & 43 & -0.47 \\
\hline 63 & 17 & 7,899 & 5.525 & 0.328 & 0.3716 & 0.0187 & 0.1078 & 0.0030 & 1905 & 51 & 2037 & 88 & 1763 & 50 & -2.18 \\
\hline 64 & 19 & 6,002 & 4.625 & 0.249 & 0.3080 & 0.0138 & 0.1089 & 0.0029 & 1754 & 45 & 1731 & 68 & 1781 & 49 & 0.62 \\
\hline 65 & 16 & 1,696 & 11.220 & 1.105 & 0.4703 & 0.0417 & 0.1730 & 0.0048 & 2542 & 92 & 2485 & 183 & 2587 & 47 & 0.59 \\
\hline 66 & 19 & 10,271 & 6.635 & 0.417 & 0.4594 & 0.0247 & 0.1047 & 0.0028 & 2064 & 55 & 2437 & 109 & 1710 & 49 & -3.36 \\
\hline 67 & 20 & 22,592 & 6.338 & 0.391 & 0.4364 & 0.0233 & 0.1053 & 0.0028 & 2024 & 54 & 2334 & 105 & 1720 & 48 & -3.25 \\
\hline 68 & 16 & 8,714 & 5.439 & 0.323 & 0.3575 & 0.0182 & 0.1103 & 0.0030 & 1891 & 51 & 1970 & 86 & 1805 & 50 & -1.47 \\
\hline 69 & 16 & 4,826 & 5.055 & 0.281 & 0.3265 & 0.0151 & 0.1123 & 0.0031 & 1829 & 47 & 1822 & 73 & 1837 & 49 & 0.16 \\
\hline 70 & 16 & 6,091 & 6.718 & 0.500 & 0.4626 & 0.0303 & 0.1053 & 0.0029 & 2075 & 66 & 2451 & 133 & 1720 & 50 & -3.33 \\
\hline 71 & 16 & 7,336 & 6.381 & 0.413 & 0.4451 & 0.0247 & 0.1040 & 0.0028 & 2030 & 57 & 2374 & 110 & 1696 & 50 & -3.39 \\
\hline 72 & 20 & 8,411 & 12.018 & 0.722 & 0.4686 & 0.0238 & 0.1860 & 0.0049 & 2606 & 56 & 2478 & 104 & 2707 & 43 & 1.40 \\
\hline
\end{tabular}


Isotopic ratios (corrected)

Ages (Ma)

\begin{tabular}{|c|c|c|c|c|c|c|c|c|c|c|c|c|c|c|c|}
\hline \# & $\mathrm{N}$ & $\frac{{ }^{206} \mathrm{~Pb}}{{ }^{204} \mathrm{~Pb}^{\dagger}}$ & $\begin{array}{c}{ }^{207} \mathrm{~Pb}^{*} \\
{ }^{235} \mathrm{U}\end{array}$ & $\begin{array}{c} \pm \\
(\mathrm{abs})\end{array}$ & $\begin{array}{l}{ }^{206} \mathrm{~Pb}^{*} \\
{ }^{238} \mathrm{U}\end{array}$ & $\begin{array}{c} \pm \\
(\mathrm{abs})\end{array}$ & $\begin{array}{l}{ }^{207} \mathrm{~Pb}^{*} \\
{ }^{206} \mathrm{~Pb}^{*}\end{array}$ & $\begin{array}{c} \pm \\
(\mathrm{abs})\end{array}$ & $\begin{array}{c}{ }^{207} \mathrm{~Pb}^{*} \\
{ }^{235} \mathrm{U}\end{array}$ & $\begin{array}{c} \pm \\
(\mathrm{abs})\end{array}$ & $\begin{array}{c}{ }^{206} \mathrm{~Pb}^{*} \\
{ }^{238} \mathrm{U} \\
\end{array}$ & $\begin{array}{c} \pm \\
(\mathrm{abs})\end{array}$ & $\begin{array}{l}{ }^{207} \mathrm{~Pb}^{*} \\
{ }^{206} \mathrm{~Pb}^{*}\end{array}$ & $\begin{array}{c} \pm \\
(\mathrm{abs})\end{array}$ & $\begin{array}{c}\text { Disc. } \\
(\%)\end{array}$ \\
\hline 73 & 20 & 7,214 & 4.522 & 0.241 & 0.3026 & 0.0134 & 0.1084 & 0.0029 & 1735 & 44 & 1704 & 67 & 1773 & 49 & 0.87 \\
\hline 74 & 20 & 2,901 & 5.015 & 0.318 & 0.3491 & 0.0185 & 0.1042 & 0.0029 & 1822 & 54 & 1930 & 88 & 1700 & 52 & -2.08 \\
\hline 75 & 14 & 5,348 & 4.694 & 0.272 & 0.3226 & 0.0155 & 0.1055 & 0.0030 & 1766 & 48 & 1802 & 76 & 1723 & 52 & -0.86 \\
\hline 76 & 11 & 5,532 & 4.018 & 0.342 & 0.2747 & 0.0213 & 0.1061 & 0.0031 & 1638 & 69 & 1564 & 108 & 1734 & 54 & 2.58 \\
\hline 77 & 14 & 6,396 & 4.328 & 0.245 & 0.2993 & 0.0143 & 0.1049 & 0.0029 & 1699 & 47 & 1688 & 71 & 1712 & 51 & 0.31 \\
\hline 78 & 19 & 11,797 & 7.214 & 0.419 & 0.4043 & 0.0200 & 0.1294 & 0.0034 & 2138 & 52 & 2189 & 92 & 2090 & 46 & -0.72 \\
\hline 79 & 15 & 3,247 & 6.474 & 0.424 & 0.4480 & 0.0242 & 0.1048 & 0.0030 & 2042 & 58 & 2386 & 108 & 1711 & 52 & -3.34 \\
\hline 80 & 16 & 5,094 & 4.914 & 0.272 & 0.3126 & 0.0144 & 0.1140 & 0.0031 & 1805 & 47 & 1754 & 71 & 1864 & 50 & 1.35 \\
\hline 81 & 19 & 10,561 & 4.164 & 0.219 & 0.2877 & 0.0127 & 0.1050 & 0.0028 & 1667 & 43 & 1630 & 64 & 1714 & 49 & 1.15 \\
\hline 82 & 18 & 9,276 & 4.531 & 0.262 & 0.3132 & 0.0156 & 0.1049 & 0.0028 & 1737 & 48 & 1756 & 76 & 1713 & 49 & -0.50 \\
\hline 83 & 19 & 6,568 & 4.020 & 0.211 & 0.2732 & 0.0120 & 0.1067 & 0.0029 & 1638 & 43 & 1557 & 61 & 1744 & 49 & 2.89 \\
\hline 84 & 18 & 6,357 & 3.615 & 0.188 & 0.2457 & 0.0107 & 0.1067 & 0.0029 & 1553 & 41 & 1416 & 55 & 1744 & 49 & 6.26 \\
\hline 85 & 14 & 4,657 & 4.224 & 0.256 & 0.2930 & 0.0151 & 0.1046 & 0.0029 & 1679 & 50 & 1656 & 75 & 1706 & 51 & 0.66 \\
\hline 86 & 18 & 8,904 & 5.481 & 0.337 & 0.3812 & 0.0202 & 0.1043 & 0.0028 & 1898 & 53 & 2082 & 94 & 1701 & 49 & -2.80 \\
\hline 87 & 20 & 10,401 & 4.300 & 0.229 & 0.2959 & 0.0133 & 0.1054 & 0.0028 & 1693 & 44 & 1671 & 66 & 1722 & 49 & 0.66 \\
\hline 88 & 16 & 3,929 & 5.032 & 0.297 & 0.3146 & 0.0156 & 0.1160 & 0.0032 & 1825 & 50 & 1763 & 77 & 1896 & 50 & 1.61 \\
\hline 89 & 15 & 3,455 & 4.008 & 0.223 & 0.2774 & 0.0127 & 0.1048 & 0.0029 & 1636 & 45 & 1578 & 64 & 1711 & 51 & 1.97 \\
\hline 90 & 12 & 2,401 & 4.773 & 0.318 & 0.3319 & 0.0184 & 0.1043 & 0.0031 & 1780 & 56 & 1848 & 89 & 1702 & 54 & -1.48 \\
\hline 91 & 13 & 3,002 & 3.687 & 0.229 & 0.2558 & 0.0135 & 0.1045 & 0.0030 & 1569 & 50 & 1468 & 69 & 1706 & 53 & 4.14 \\
\hline 92 & 19 & 9,373 & 4.428 & 0.234 & 0.2969 & 0.0131 & 0.1082 & 0.0029 & 1718 & 44 & 1676 & 65 & 1769 & 49 & 1.22 \\
\hline 93 & 19 & 17,732 & 5.799 & 0.404 & 0.3978 & 0.0249 & 0.1057 & 0.0028 & 1946 & 60 & 2159 & 115 & 1727 & 48 & -2.89 \\
\hline 94 & 20 & 11,764 & 4.251 & 0.223 & 0.2871 & 0.0127 & 0.1074 & 0.0028 & 1684 & 43 & 1627 & 63 & 1755 & 48 & 1.80 \\
\hline 95 & 15 & 2,575 & 4.087 & 0.231 & 0.2826 & 0.0130 & 0.1049 & 0.0030 & 1652 & 46 & 1604 & 65 & 1713 & 52 & 1.55 \\
\hline 96 & 19 & 11,513 & 4.239 & 0.222 & 0.2903 & 0.0128 & 0.1059 & 0.0028 & 1682 & 43 & 1643 & 64 & 1730 & 48 & 1.18 \\
\hline 97 & 20 & 8,937 & 4.195 & 0.221 & 0.2822 & 0.0125 & 0.1078 & 0.0029 & 1673 & 43 & 1603 & 63 & 1763 & 48 & 2.34 \\
\hline 98 & 20 & 7,219 & 4.462 & 0.254 & 0.2827 & 0.0138 & 0.1145 & 0.0031 & 1724 & 47 & 1605 & 69 & 1872 & 48 & 4.04 \\
\hline 99 & 19 & 8,160 & 4.335 & 0.234 & 0.2990 & 0.0136 & 0.1052 & 0.0028 & 1700 & 45 & 1686 & 68 & 1717 & 49 & 0.40 \\
\hline 100 & 20 & 8,918 & 4.554 & 0.241 & 0.3077 & 0.0137 & 0.1073 & 0.0028 & 1741 & 44 & 1729 & 67 & 1755 & 48 & 0.31 \\
\hline 101 & 20 & 658 & 3.641 & 0.318 & 0.2470 & 0.0168 & 0.1069 & 0.0034 & 1559 & 70 & 1423 & 87 & 1748 & 59 & 6.15 \\
\hline 102 & 19 & 11,264 & 16.286 & 1.009 & 0.5050 & 0.0265 & 0.2339 & 0.0061 & 2894 & 59 & 2635 & 113 & 3079 & 42 & 2.52 \\
\hline
\end{tabular}

\begin{tabular}{cccccccccccccccc}
\multicolumn{10}{c}{ Sample J01-BR2: Basal conglomerate, Blue Ridge, Colorado } \\
1 & 17 & 3,942 & 3.642 & 0.203 & 0.2502 & 0.0115 & 0.1056 & 0.0029 & 1559 & 44 & 1440 & 59 & 1724 & 51 & 5.21 \\
2 & 16 & 6,383 & 4.272 & 0.252 & 0.2943 & 0.0147 & 0.1053 & 0.0029 & 1688 & 49 & 1663 & 73 & 1719 & 50 & 0.74 \\
3 & 20 & 14,401 & 4.551 & 0.273 & 0.3138 & 0.0163 & 0.1052 & 0.0028 & 1740 & 50 & 1759 & 80 & 1718 & 48 & -0.48 \\
4 & 19 & 10,163 & 4.670 & 0.288 & 0.3213 & 0.0172 & 0.1054 & 0.0028 & 1762 & 52 & 1796 & 84 & 1721 & 49 & -0.82 \\
5 & 20 & 13,584 & 4.433 & 0.253 & 0.3044 & 0.0147 & 0.1056 & 0.0028 & 1719 & 47 & 1713 & 73 & 1725 & 49 & 0.15 \\
6 & 15 & 3,005 & 3.801 & 0.247 & 0.2587 & 0.0142 & 0.1066 & 0.0030 & 1593 & 52 & 1483 & 73 & 1741 & 52 & 4.45 \\
7 & 18 & 14,581 & 5.413 & 0.434 & 0.3720 & 0.0273 & 0.1055 & 0.0028 & 1887 & 69 & 2039 & 128 & 1724 & 49 & -2.48 \\
8 & 17 & 9,236 & 3.695 & 0.205 & 0.2551 & 0.0118 & 0.1051 & 0.0029 & 1570 & 44 & 1465 & 61 & 1716 & 50 & 4.41 \\
9 & 10 & 4,319 & 4.077 & 0.295 & 0.2785 & 0.0175 & 0.1062 & 0.0031 & 1650 & 59 & 1584 & 88 & 1735 & 54 & 2.24 \\
10 & 20 & 13,658 & 4.667 & 0.268 & 0.3211 & 0.0156 & 0.1054 & 0.0028 & 1761 & 48 & 1795 & 76 & 1721 & 49 & -0.81 \\
11 & 18 & 21,021 & 3.948 & 0.215 & 0.2710 & 0.0125 & 0.1057 & 0.0028 & 1624 & 44 & 1546 & 63 & 1726 & 49 & 2.82 \\
12 & 18 & 12,981 & 4.314 & 0.257 & 0.2977 & 0.0153 & 0.1051 & 0.0028 & 1696 & 49 & 1680 & 76 & 1716 & 49 & 0.47 \\
13 & 19 & 25,248 & 4.539 & 0.263 & 0.3108 & 0.0154 & 0.1059 & 0.0028 & 1738 & 48 & 1745 & 76 & 1730 & 49 & -0.17 \\
14 & 11 & 3,019 & 4.246 & 0.362 & 0.2917 & 0.0221 & 0.1056 & 0.0031 & 1683 & 70 & 1650 & 110 & 1724 & 54 & 0.99
\end{tabular}


Isotopic ratios (corrected)

Ages (Ma)

\begin{tabular}{|c|c|c|c|c|c|c|c|c|c|c|c|c|c|c|c|}
\hline$\#$ & $\mathrm{~N}$ & $\frac{{ }^{206} \mathrm{~Pb}}{{ }^{204} \mathrm{~Pb}^{\dagger}}$ & $\begin{array}{l}{ }^{207} \mathrm{~Pb}^{*} \\
{ }^{235} \mathrm{U}\end{array}$ & $\begin{array}{c} \pm \\
\text { (abs) }\end{array}$ & $\begin{array}{l}{ }^{206} \mathrm{~Pb}^{*} \\
{ }^{238} \mathrm{U}\end{array}$ & $\begin{array}{c} \pm \\
\text { (abs) }\end{array}$ & $\begin{array}{l}{ }^{207} \mathrm{~Pb}^{*} \\
{ }^{206} \mathrm{~Pb}^{*}\end{array}$ & $\begin{array}{c} \pm \\
(\mathrm{abs})\end{array}$ & $\begin{array}{c}{ }^{207} \mathrm{~Pb}^{*} \\
{ }^{235} \mathrm{U}\end{array}$ & $\begin{array}{c} \pm \\
(\mathrm{abs})\end{array}$ & $\begin{array}{c}{ }^{206} \mathrm{~Pb}^{*} \\
{ }^{238} \mathrm{U}\end{array}$ & $\begin{array}{c} \pm \\
(\mathrm{abs})\end{array}$ & $\begin{array}{l}{ }^{207} \mathrm{~Pb}^{*} \\
{ }^{206} \mathrm{~Pb}^{*}\end{array}$ & $\begin{array}{c} \pm \\
(\mathrm{abs})\end{array}$ & $\begin{array}{c}\text { Disc. } \\
(\%)\end{array}$ \\
\hline 15 & 19 & 13,415 & 3.935 & 0.216 & 0.2715 & 0.0125 & 0.1051 & 0.0028 & 1621 & 44 & 1548 & 64 & 1717 & 49 & 2.62 \\
\hline 16 & 19 & 14,287 & 4.405 & 0.259 & 0.3057 & 0.0154 & 0.1045 & 0.0028 & 1713 & 49 & 1719 & 76 & 1706 & 49 & -0.16 \\
\hline 17 & 20 & 6,039 & 4.080 & 0.231 & 0.2801 & 0.0131 & 0.1056 & 0.0029 & 1650 & 46 & 1592 & 66 & 1726 & 51 & 1.96 \\
\hline 18 & 16 & 1,507 & 4.365 & 0.284 & 0.2929 & 0.0150 & 0.1081 & 0.0033 & 1706 & 54 & 1656 & 75 & 1767 & 56 & 1.50 \\
\hline 19 & 20 & 3,971 & 3.373 & 0.235 & 0.2309 & 0.0142 & 0.1060 & 0.0029 & 1498 & 55 & 1339 & 74 & 1731 & 50 & 8.37 \\
\hline 20 & 17 & 14,352 & 4.145 & 0.231 & 0.2840 & 0.0134 & 0.1058 & 0.0028 & 1663 & 46 & 1612 & 67 & 1729 & 49 & 1.67 \\
\hline 21 & 17 & 11,168 & 4.021 & 0.223 & 0.2783 & 0.0130 & 0.1048 & 0.0028 & 1638 & 45 & 1583 & 65 & 1710 & 49 & 1.88 \\
\hline 22 & 19 & 50,968 & 3.949 & 0.213 & 0.2703 & 0.0124 & 0.1060 & 0.0028 & 1624 & 44 & 1542 & 63 & 1731 & 48 & 2.98 \\
\hline 23 & 16 & 2,069 & 3.184 & 0.342 & 0.2168 & 0.0211 & 0.1065 & 0.0031 & 1453 & 83 & 1265 & 112 & 1740 & 53 & 11.43 \\
\hline 24 & 16 & 15,531 & 3.977 & 0.229 & 0.2723 & 0.0134 & 0.1059 & 0.0029 & 1630 & 47 & 1553 & 68 & 1730 & 49 & 2.76 \\
\hline 25 & 14 & 6,144 & 4.208 & 0.256 & 0.2895 & 0.0149 & 0.1054 & 0.0029 & 1676 & 50 & 1639 & 75 & 1722 & 51 & 1.13 \\
\hline 26 & 5 & 11,599 & 3.860 & 0.281 & 0.2658 & 0.0162 & 0.1053 & 0.0038 & 1605 & 59 & 1520 & 83 & 1720 & 66 & 3.25 \\
\hline 27 & 20 & 9,782 & 4.356 & 0.244 & 0.3008 & 0.0141 & 0.1050 & 0.0028 & 1704 & 46 & 1695 & 70 & 1715 & 49 & 0.25 \\
\hline 28 & 20 & 31,981 & 3.818 & 0.205 & 0.2617 & 0.0118 & 0.1058 & 0.0028 & 1597 & 43 & 1499 & 60 & 1728 & 49 & 3.85 \\
\hline 29 & 19 & 17,715 & 4.118 & 0.225 & 0.2830 & 0.0131 & 0.1055 & 0.0028 & 1658 & 45 & 1606 & 66 & 1724 & 48 & 1.68 \\
\hline 30 & 20 & 6,957 & 3.913 & 0.216 & 0.2697 & 0.0124 & 0.1052 & 0.0029 & 1616 & 45 & 1539 & 63 & 1718 & 50 & 2.81 \\
\hline 31 & 20 & 15,928 & 3.950 & 0.214 & 0.2714 & 0.0124 & 0.1056 & 0.0028 & 1624 & 44 & 1548 & 63 & 1724 & 48 & 2.76 \\
\hline 32 & 18 & 13,950 & 4.133 & 0.229 & 0.2842 & 0.0133 & 0.1055 & 0.0028 & 1661 & 45 & 1613 & 67 & 1723 & 49 & 1.56 \\
\hline 33 & 19 & 11,922 & 3.925 & 0.215 & 0.2711 & 0.0125 & 0.1050 & 0.0028 & 1619 & 44 & 1546 & 63 & 1714 & 49 & 2.62 \\
\hline 34 & 9 & 5,165 & 4.266 & 0.277 & 0.2926 & 0.0159 & 0.1058 & 0.0032 & 1687 & 53 & 1654 & 79 & 1727 & 56 & 0.98 \\
\hline 35 & 20 & 14,777 & 4.273 & 0.241 & 0.2938 & 0.0140 & 0.1055 & 0.0028 & 1688 & 46 & 1661 & 70 & 1723 & 49 & 0.82 \\
\hline 36 & 16 & 7,951 & 3.874 & 0.219 & 0.2681 & 0.0127 & 0.1048 & 0.0029 & 1608 & 46 & 1531 & 65 & 1711 & 50 & 2.86 \\
\hline 37 & 17 & 12,232 & 4.171 & 0.241 & 0.2857 & 0.0140 & 0.1059 & 0.0029 & 1668 & 47 & 1620 & 70 & 1730 & 50 & 1.54 \\
\hline 38 & 19 & 11,140 & 3.899 & 0.212 & 0.2675 & 0.0122 & 0.1057 & 0.0028 & 1614 & 44 & 1528 & 62 & 1727 & 49 & 3.19 \\
\hline 39 & 18 & 14,768 & 3.958 & 0.216 & 0.2724 & 0.0125 & 0.1054 & 0.0028 & 1626 & 44 & 1553 & 64 & 1721 & 49 & 2.59 \\
\hline 40 & 19 & 9,135 & 4.241 & 0.240 & 0.2906 & 0.0139 & 0.1059 & 0.0028 & 1682 & 47 & 1644 & 69 & 1729 & 49 & 1.15 \\
\hline 41 & 18 & 14,992 & 3.696 & 0.200 & 0.2544 & 0.0116 & 0.1054 & 0.0028 & 1571 & 43 & 1461 & 59 & 1721 & 49 & 4.61 \\
\hline 42 & 19 & 13,816 & 4.449 & 0.277 & 0.3065 & 0.0167 & 0.1053 & 0.0028 & 1721 & 52 & 1723 & 82 & 1719 & 48 & -0.05 \\
\hline 43 & 19 & 7,391 & 4.139 & 0.234 & 0.2845 & 0.0134 & 0.1055 & 0.0029 & 1662 & 46 & 1614 & 67 & 1724 & 50 & 1.56 \\
\hline 44 & 19 & 14,922 & 3.874 & 0.210 & 0.2661 & 0.0122 & 0.1056 & 0.0028 & 1608 & 44 & 1521 & 62 & 1724 & 49 & 3.29 \\
\hline 45 & 19 & 35,148 & 3.864 & 0.208 & 0.2649 & 0.0120 & 0.1058 & 0.0028 & 1606 & 43 & 1515 & 61 & 1728 & 49 & 3.50 \\
\hline 46 & 19 & 5,704 & 4.128 & 0.236 & 0.2867 & 0.0136 & 0.1044 & 0.0029 & 1660 & 47 & 1625 & 68 & 1704 & 51 & 1.10 \\
\hline 47 & 18 & 4,879 & 4.356 & 0.444 & 0.2993 & 0.0284 & 0.1056 & 0.0029 & 1704 & 84 & 1688 & 141 & 1724 & 50 & 0.47 \\
\hline 48 & 13 & 3,792 & 3.868 & 0.229 & 0.2667 & 0.0130 & 0.1052 & 0.0030 & 1607 & 48 & 1524 & 66 & 1718 & 53 & 3.13 \\
\hline 49 & 20 & 6,550 & 4.075 & 0.229 & 0.2789 & 0.0130 & 0.1060 & 0.0029 & 1649 & 46 & 1586 & 65 & 1731 & 51 & 2.14 \\
\hline 50 & 20 & 13,735 & 4.050 & 0.226 & 0.2798 & 0.0133 & 0.1050 & 0.0028 & 1644 & 46 & 1590 & 67 & 1714 & 49 & 1.80 \\
\hline 51 & 18 & 10,020 & 3.997 & 0.223 & 0.2756 & 0.0129 & 0.1052 & 0.0028 & 1634 & 45 & 1569 & 65 & 1717 & 49 & 2.23 \\
\hline 52 & 14 & 2,478 & 4.389 & 0.288 & 0.3018 & 0.0164 & 0.1055 & 0.0030 & 1710 & 54 & 1700 & 81 & 1723 & 53 & 0.28 \\
\hline 53 & 18 & 18,525 & 4.169 & 0.231 & 0.2879 & 0.0135 & 0.1050 & 0.0028 & 1668 & 45 & 1631 & 68 & 1715 & 49 & 1.15 \\
\hline 54 & 18 & 4,043 & 3.231 & 0.181 & 0.2211 & 0.0103 & 0.1060 & 0.0029 & 1465 & 43 & 1288 & 54 & 1731 & 50 & 10.25 \\
\hline 55 & 12 & 5,994 & 3.406 & 0.250 & 0.2348 & 0.0154 & 0.1052 & 0.0030 & 1506 & 58 & 1360 & 80 & 1718 & 52 & 7.37 \\
\hline 56 & 16 & 5,310 & 4.014 & 0.234 & 0.2760 & 0.0136 & 0.1055 & 0.0029 & 1637 & 47 & 1571 & 69 & 1722 & 50 & 2.27 \\
\hline 57 & 15 & 3,025 & 3.729 & 0.229 & 0.2564 & 0.0130 & 0.1055 & 0.0030 & 1578 & 49 & 1471 & 67 & 1723 & 53 & 4.38 \\
\hline 58 & 10 & 3,257 & 3.366 & 0.527 & 0.2314 & 0.0341 & 0.1055 & 0.0032 & 1497 & 122 & 1342 & 178 & 1723 & 56 & 8.09 \\
\hline 59 & 15 & 4,151 & 4.055 & 0.243 & 0.2789 & 0.0139 & 0.1054 & 0.0030 & 1645 & 49 & 1586 & 70 & 1722 & 52 & 2.01 \\
\hline 60 & 11 & 5,535 & 3.663 & 0.270 & 0.2522 & 0.0164 & 0.1053 & 0.0031 & 1563 & 59 & 1450 & 85 & 1720 & 54 & 4.87 \\
\hline
\end{tabular}


Isotopic ratios (corrected)

Ages (Ma)

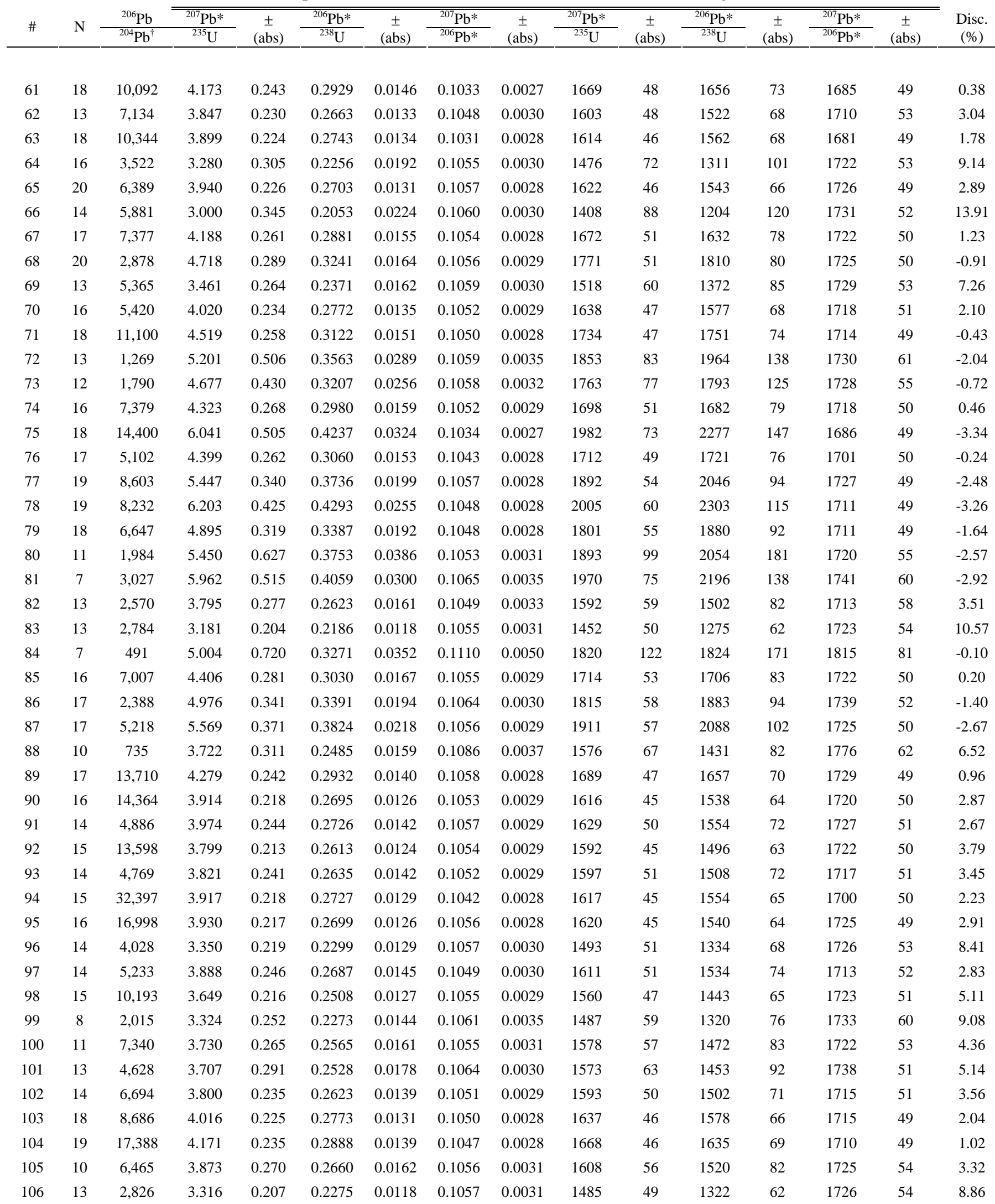


Isotopic ratios (corrected)

Ages (Ma)

\begin{tabular}{|c|c|c|c|c|c|c|c|c|c|c|c|c|c|c|c|}
\hline \# & $\mathrm{N}$ & $\frac{{ }^{206} \mathrm{~Pb}}{{ }^{204} \mathrm{~Pb}^{\dagger}}$ & $\begin{array}{c}{ }^{207} \mathrm{~Pb}^{*} \\
{ }^{235} \mathrm{U}\end{array}$ & $\begin{array}{c} \pm \\
(\mathrm{abs})\end{array}$ & $\begin{array}{c}{ }^{206} \mathrm{~Pb}^{*} \\
{ }^{238} \mathrm{U}\end{array}$ & $\begin{array}{c} \pm \\
(\mathrm{abs})\end{array}$ & $\begin{array}{l}{ }^{207} \mathrm{~Pb}^{*} \\
{ }^{206} \mathrm{~Pb}^{*}\end{array}$ & $\begin{array}{c} \pm \\
(\mathrm{abs})\end{array}$ & $\begin{array}{c}{ }^{207} \mathrm{~Pb}^{*} \\
{ }^{235} \mathrm{U}\end{array}$ & $\begin{array}{c} \pm \\
(\mathrm{abs})\end{array}$ & $\begin{array}{c}{ }^{206} \mathrm{~Pb}^{*} \\
{ }^{238} \mathrm{U}\end{array}$ & $\begin{array}{c} \pm \\
\text { (abs) }\end{array}$ & $\begin{array}{l}{ }^{207} \mathrm{~Pb}^{*} \\
{ }^{206} \mathrm{~Pb}^{*}\end{array}$ & $\begin{array}{c} \pm \\
\text { (abs) }\end{array}$ & $\begin{array}{c}\text { Disc. } \\
(\%)\end{array}$ \\
\hline 107 & 15 & 4,488 & 3.615 & 0.236 & 0.2495 & 0.0141 & 0.1051 & 0.0029 & 1553 & 52 & 1436 & 73 & 1716 & 51 & 5.15 \\
\hline 108 & 19 & 22,727 & 4.975 & 0.316 & 0.3437 & 0.0192 & 0.1050 & 0.0028 & 1815 & 54 & 1904 & 92 & 1714 & 48 & -1.79 \\
\hline 109 & 19 & 12,518 & 4.170 & 0.237 & 0.2889 & 0.0138 & 0.1047 & 0.0029 & 1668 & 47 & 1636 & 69 & 1709 & 50 & 1.00 \\
\hline
\end{tabular}

\begin{tabular}{|c|c|c|c|c|c|c|c|c|c|c|c|c|c|c|c|}
\hline 1 & 20 & 14,994 & 4.181 & 0.268 & 0.2886 & 0.0158 & 0.1051 & 0.0027 & 1670 & 52 & 1635 & 79 & 1715 & 48 & -1.11 \\
\hline 2 & 19 & 13,218 & 3.985 & 0.248 & 0.2766 & 0.0146 & 0.1045 & 0.0027 & 1631 & 51 & 1574 & 74 & 1705 & 48 & -1.95 \\
\hline 3 & 6 & 8,810 & 4.146 & 0.322 & 0.2885 & 0.0188 & 0.1042 & 0.0034 & 1663 & 64 & 1634 & 94 & 1701 & 61 & -0.91 \\
\hline 4 & 19 & 8,161 & 4.208 & 0.269 & 0.2894 & 0.0156 & 0.1055 & 0.0028 & 1676 & 52 & 1639 & 78 & 1722 & 49 & -1.14 \\
\hline 5 & 20 & 24,012 & 3.987 & 0.249 & 0.2760 & 0.0147 & 0.1048 & 0.0027 & 1632 & 51 & 1571 & 74 & 1710 & 48 & -2.09 \\
\hline 6 & 20 & 20,788 & 3.770 & 0.230 & 0.2610 & 0.0135 & 0.1048 & 0.0027 & 1586 & 49 & 1495 & 69 & 1710 & 48 & -3.62 \\
\hline 7 & 18 & 17,187 & 3.857 & 0.242 & 0.2707 & 0.0144 & 0.1033 & 0.0027 & 1605 & 51 & 1544 & 73 & 1685 & 49 & -2.17 \\
\hline 8 & 19 & 19,166 & 4.314 & 0.276 & 0.3027 & 0.0165 & 0.1033 & 0.0027 & 1696 & 53 & 1705 & 82 & 1685 & 49 & 0.24 \\
\hline 9 & 19 & 16,518 & 4.299 & 0.274 & 0.3001 & 0.0162 & 0.1039 & 0.0027 & 1693 & 53 & 1692 & 80 & 1695 & 48 & -0.04 \\
\hline 10 & 19 & 11,042 & 4.225 & 0.267 & 0.2916 & 0.0156 & 0.1051 & 0.0028 & 1679 & 52 & 1650 & 78 & 1716 & 49 & -0.88 \\
\hline 11 & 16 & 10,662 & 4.282 & 0.288 & 0.3001 & 0.0172 & 0.1035 & 0.0028 & 1690 & 55 & 1692 & 85 & 1688 & 50 & 0.05 \\
\hline 12 & 19 & 11,217 & 4.752 & 0.313 & 0.3289 & 0.0184 & 0.1048 & 0.0028 & 1776 & 55 & 1833 & 89 & 1711 & 49 & 1.27 \\
\hline 13 & 19 & 11,591 & 4.775 & 0.331 & 0.3350 & 0.0201 & 0.1034 & 0.0027 & 1781 & 58 & 1863 & 97 & 1686 & 49 & 1.75 \\
\hline 14 & 15 & 8,969 & 4.954 & 0.338 & 0.3404 & 0.0196 & 0.1056 & 0.0029 & 1812 & 58 & 1889 & 94 & 1724 & 50 & 1.59 \\
\hline 15 & 19 & 14,217 & 4.872 & 0.330 & 0.3369 & 0.0194 & 0.1049 & 0.0028 & 1797 & 57 & 1872 & 94 & 1712 & 49 & 1.57 \\
\hline 16 & 19 & 12,756 & 5.416 & 0.378 & 0.3770 & 0.0224 & 0.1042 & 0.0028 & 1887 & 60 & 2062 & 105 & 1700 & 49 & 2.74 \\
\hline 17 & 18 & 15,578 & 3.864 & 0.240 & 0.2682 & 0.0141 & 0.1045 & 0.0028 & 1606 & 50 & 1532 & 72 & 1705 & 49 & -2.75 \\
\hline 18 & 19 & 19,665 & 3.777 & 0.232 & 0.2630 & 0.0137 & 0.1042 & 0.0027 & 1588 & 49 & 1505 & 70 & 1700 & 49 & -3.20 \\
\hline 19 & 19 & 20,643 & 3.949 & 0.248 & 0.2748 & 0.0147 & 0.1042 & 0.0027 & 1624 & 51 & 1565 & 74 & 1701 & 48 & -2.05 \\
\hline 20 & 19 & 6,093 & 4.164 & 0.266 & 0.2876 & 0.0154 & 0.1050 & 0.0028 & 1667 & 52 & 1629 & 77 & 1715 & 49 & -1.18 \\
\hline 21 & 18 & 6,179 & 3.903 & 0.246 & 0.2716 & 0.0143 & 0.1042 & 0.0028 & 1614 & 51 & 1549 & 73 & 1701 & 50 & -2.35 \\
\hline 22 & 19 & 24,391 & 3.826 & 0.236 & 0.2657 & 0.0139 & 0.1045 & 0.0027 & 1598 & 50 & 1519 & 71 & 1705 & 48 & -3.00 \\
\hline 23 & 20 & 11,017 & 4.806 & 0.306 & 0.3051 & 0.0164 & 0.1143 & 0.0030 & 1786 & 54 & 1716 & 81 & 1868 & 47 & -1.96 \\
\hline 24 & 20 & 15,164 & 4.518 & 0.291 & 0.3125 & 0.0171 & 0.1049 & 0.0028 & 1734 & 54 & 1753 & 84 & 1712 & 48 & 0.47 \\
\hline 25 & 19 & 10,462 & 4.279 & 0.275 & 0.2966 & 0.0160 & 0.1047 & 0.0028 & 1689 & 53 & 1674 & 79 & 1708 & 50 & -0.44 \\
\hline 26 & 19 & 15,710 & 4.042 & 0.254 & 0.2827 & 0.0151 & 0.1037 & 0.0027 & 1643 & 51 & 1605 & 76 & 1691 & 48 & -1.22 \\
\hline 27 & 20 & 20,239 & 3.996 & 0.252 & 0.2761 & 0.0148 & 0.1050 & 0.0027 & 1633 & 51 & 1572 & 75 & 1714 & 48 & -2.13 \\
\hline 28 & 20 & 11,316 & 4.202 & 0.267 & 0.2929 & 0.0157 & 0.1040 & 0.0027 & 1674 & 52 & 1656 & 78 & 1697 & 49 & -0.55 \\
\hline 29 & 20 & 16,586 & 3.850 & 0.239 & 0.2678 & 0.0140 & 0.1043 & 0.0028 & 1603 & 50 & 1530 & 71 & 1701 & 49 & -2.72 \\
\hline 30 & 19 & 30,639 & 4.373 & 0.278 & 0.2924 & 0.0158 & 0.1085 & 0.0028 & 1707 & 53 & 1653 & 79 & 1774 & 48 & -1.64 \\
\hline 31 & 20 & 31,880 & 4.661 & 0.296 & 0.3110 & 0.0168 & 0.1087 & 0.0028 & 1760 & 53 & 1746 & 83 & 1778 & 47 & -0.39 \\
\hline 32 & 20 & 12,164 & 4.871 & 0.325 & 0.3390 & 0.0193 & 0.1042 & 0.0027 & 1797 & 56 & 1882 & 93 & 1700 & 48 & 1.75 \\
\hline 33 & 18 & 14,014 & 5.098 & 0.579 & 0.3571 & 0.0383 & 0.1035 & 0.0028 & 1836 & 96 & 1968 & 182 & 1688 & 49 & 2.39 \\
\hline 34 & 19 & 10,885 & 4.509 & 0.292 & 0.3132 & 0.0172 & 0.1044 & 0.0028 & 1733 & 54 & 1757 & 84 & 1704 & 49 & 0.61 \\
\hline 35 & 18 & 11,334 & 4.227 & 0.272 & 0.2947 & 0.0161 & 0.1040 & 0.0028 & 1679 & 53 & 1665 & 80 & 1697 & 49 & -0.43 \\
\hline 36 & 18 & 15,123 & 4.057 & 0.255 & 0.2819 & 0.0150 & 0.1044 & 0.0028 & 1646 & 51 & 1601 & 75 & 1703 & 49 & -1.47 \\
\hline 37 & 19 & 14,722 & 4.504 & 0.289 & 0.3137 & 0.0170 & 0.1041 & 0.0027 & 1732 & 53 & 1759 & 83 & 1699 & 48 & 0.68 \\
\hline 38 & 19 & 10,251 & 3.983 & 0.250 & 0.2767 & 0.0146 & 0.1044 & 0.0028 & 1631 & 51 & 1575 & 74 & 1704 & 49 & -1.92 \\
\hline 39 & 20 & 13,650 & 3.990 & 0.251 & 0.2776 & 0.0148 & 0.1042 & 0.0028 & 1632 & 51 & 1579 & 75 & 1701 & 49 & -1.80 \\
\hline 40 & 19 & 8,306 & 4.546 & 0.298 & 0.3166 & 0.0176 & 0.1041 & 0.0028 & 1739 & 55 & 1773 & 86 & 1699 & 49 & 0.83 \\
\hline 41 & 19 & 11,816 & 4.910 & 0.336 & 0.3444 & 0.0202 & 0.1034 & 0.0027 & 1804 & 58 & 1908 & 97 & 1686 & 49 & 2.06 \\
\hline
\end{tabular}


Isotopic ratios (corrected)

Ages (Ma)

\begin{tabular}{|c|c|c|c|c|c|c|c|c|c|c|c|c|c|c|c|}
\hline$\#$ & $\mathrm{~N}$ & $\frac{{ }^{206} \mathrm{~Pb}}{{ }^{204} \mathrm{~Pb}^{\dagger}}$ & $\begin{array}{c}{ }^{207} \mathrm{~Pb}^{*} \\
{ }^{235} \mathrm{U}\end{array}$ & $\begin{array}{c} \pm \\
\text { (abs) }\end{array}$ & $\begin{array}{l}{ }^{206} \mathrm{~Pb}^{*} \\
{ }^{238} \mathrm{U}\end{array}$ & $\begin{array}{c} \pm \\
(\mathrm{abs})\end{array}$ & $\begin{array}{l}{ }^{207} \mathrm{~Pb}^{*} \\
{ }^{206} \mathrm{~Pb}^{*}\end{array}$ & $\begin{array}{c} \pm \\
\text { (abs) }\end{array}$ & $\begin{array}{c}{ }^{207} \mathrm{~Pb}^{*} \\
{ }^{235} \mathrm{U}\end{array}$ & $\begin{array}{c} \pm \\
\text { (abs) }\end{array}$ & $\begin{array}{c}{ }^{206} \mathrm{~Pb}^{*} \\
{ }^{238} \mathrm{U}\end{array}$ & $\begin{array}{c} \pm \\
(\mathrm{abs})\end{array}$ & $\begin{array}{l}{ }^{207} \mathrm{~Pb}^{*} \\
{ }^{206} \mathrm{~Pb}^{*}\end{array}$ & $\begin{array}{c} \pm \\
(\mathrm{abs})\end{array}$ & $\begin{array}{c}\text { Disc. } \\
\text { (\%) }\end{array}$ \\
\hline 42 & 14 & 4,971 & 4.731 & 0.325 & 0.3269 & 0.0189 & 0.1049 & 0.0029 & 1773 & 58 & 1823 & 92 & 1713 & 51 & 1.16 \\
\hline 43 & 20 & 4,018 & 3.983 & 0.259 & 0.2826 & 0.0149 & 0.1022 & 0.0030 & 1631 & 53 & 1605 & 75 & 1664 & 54 & -0.84 \\
\hline 44 & 20 & 10,399 & 3.866 & 0.240 & 0.2696 & 0.0141 & 0.1040 & 0.0027 & 1607 & 50 & 1539 & 72 & 1697 & 49 & -2.48 \\
\hline 45 & 20 & 22,597 & 3.851 & 0.236 & 0.2679 & 0.0139 & 0.1043 & 0.0027 & 1603 & 49 & 1530 & 71 & 1701 & 48 & -2.72 \\
\hline 46 & 18 & 11,877 & 3.858 & 0.241 & 0.2693 & 0.0142 & 0.1039 & 0.0028 & 1605 & 50 & 1537 & 72 & 1695 & 49 & -2.46 \\
\hline 47 & 20 & 11,274 & 4.050 & 0.254 & 0.2826 & 0.0150 & 0.1040 & 0.0027 & 1644 & 51 & 1604 & 75 & 1696 & 48 & -1.30 \\
\hline 48 & 19 & 9,717 & 4.290 & 0.274 & 0.2982 & 0.0161 & 0.1043 & 0.0028 & 1691 & 53 & 1683 & 80 & 1702 & 49 & -0.25 \\
\hline 49 & 19 & 12,728 & 3.985 & 0.250 & 0.2777 & 0.0146 & 0.1041 & 0.0028 & 1631 & 51 & 1580 & 74 & 1698 & 50 & -1.75 \\
\hline 50 & 18 & 13,340 & 3.961 & 0.249 & 0.2774 & 0.0147 & 0.1035 & 0.0027 & 1626 & 51 & 1578 & 74 & 1689 & 49 & -1.62 \\
\hline 51 & 20 & 61,588 & 11.770 & 0.901 & 0.4626 & 0.0303 & 0.1845 & 0.0048 & 2586 & 72 & 2451 & 133 & 2694 & 43 & -1.53 \\
\hline 52 & 9 & 4,752 & 4.876 & 0.472 & 0.3342 & 0.0285 & 0.1058 & 0.0035 & 1798 & 82 & 1859 & 138 & 1728 & 61 & 1.31 \\
\hline 53 & 19 & 12,443 & 6.126 & 0.453 & 0.4285 & 0.0269 & 0.1037 & 0.0027 & 1994 & 65 & 2299 & 122 & 1691 & 49 & 3.34 \\
\hline 54 & 18 & 20,600 & 5.598 & 0.426 & 0.3920 & 0.0261 & 0.1036 & 0.0027 & 1916 & 66 & 2132 & 121 & 1689 & 49 & 3.04 \\
\hline 55 & 12 & 15,384 & 4.304 & 0.295 & 0.2987 & 0.0175 & 0.1045 & 0.0029 & 1694 & 57 & 1685 & 87 & 1706 & 51 & -0.26 \\
\hline 56 & 20 & 24,333 & 4.442 & 0.283 & 0.3080 & 0.0167 & 0.1046 & 0.0027 & 1720 & 53 & 1731 & 82 & 1707 & 48 & 0.28 \\
\hline 57 & 8 & 266 & 5.627 & 0.569 & 0.3015 & 0.0224 & 0.1354 & 0.0050 & 1920 & 87 & 1699 & 111 & 2169 & 65 & -6.89 \\
\hline 58 & 18 & 5,013 & 4.670 & 0.327 & 0.3253 & 0.0187 & 0.1041 & 0.0031 & 1762 & 58 & 1816 & 91 & 1699 & 55 & 1.24 \\
\hline 59 & 20 & 28,000 & 4.686 & 0.309 & 0.3158 & 0.0179 & 0.1076 & 0.0028 & 1765 & 55 & 1769 & 88 & 1759 & 48 & 0.11 \\
\hline 60 & 16 & 7,856 & 5.149 & 0.371 & 0.3622 & 0.0223 & 0.1031 & 0.0028 & 1844 & 61 & 1993 & 105 & 1681 & 50 & 2.57 \\
\hline 61 & 19 & 7,192 & 4.876 & 0.332 & 0.3403 & 0.0195 & 0.1039 & 0.0028 & 1798 & 57 & 1888 & 94 & 1695 & 51 & 1.84 \\
\hline 62 & 20 & 15,892 & 4.221 & 0.266 & 0.2928 & 0.0156 & 0.1046 & 0.0027 & 1678 & 52 & 1656 & 78 & 1707 & 48 & -0.68 \\
\hline 63 & 14 & 14,596 & 4.425 & 0.297 & 0.3070 & 0.0176 & 0.1045 & 0.0028 & 1717 & 56 & 1726 & 87 & 1706 & 50 & 0.24 \\
\hline 64 & 19 & 10,632 & 4.566 & 0.300 & 0.3185 & 0.0177 & 0.1040 & 0.0028 & 1743 & 55 & 1782 & 87 & 1696 & 49 & 0.95 \\
\hline 65 & 19 & 8,015 & 4.659 & 0.311 & 0.3251 & 0.0185 & 0.1039 & 0.0028 & 1760 & 56 & 1815 & 90 & 1695 & 49 & 1.26 \\
\hline 66 & 20 & 16,461 & 4.847 & 0.323 & 0.3240 & 0.0185 & 0.1085 & 0.0028 & 1793 & 56 & 1809 & 90 & 1775 & 48 & 0.37 \\
\hline 67 & 20 & 13,759 & 4.167 & 0.262 & 0.2887 & 0.0154 & 0.1047 & 0.0028 & 1668 & 52 & 1635 & 77 & 1709 & 48 & -1.01 \\
\hline 68 & 19 & 12,332 & 4.196 & 0.266 & 0.2910 & 0.0156 & 0.1046 & 0.0028 & 1673 & 52 & 1646 & 78 & 1707 & 49 & -0.81 \\
\hline 69 & 20 & 21,416 & 5.210 & 0.374 & 0.3645 & 0.0227 & 0.1037 & 0.0027 & 1854 & 61 & 2004 & 107 & 1691 & 48 & 2.55 \\
\hline 70 & 19 & 5,998 & 4.111 & 0.261 & 0.2878 & 0.0153 & 0.1036 & 0.0028 & 1657 & 52 & 1631 & 77 & 1690 & 50 & -0.81 \\
\hline 72 & 20 & 14,604 & 4.177 & 0.264 & 0.2909 & 0.0155 & 0.1041 & 0.0028 & 1669 & 52 & 1646 & 78 & 1699 & 49 & -0.71 \\
\hline 73 & 19 & 12,742 & 4.354 & 0.272 & 0.2821 & 0.0149 & 0.1120 & 0.0029 & 1704 & 52 & 1602 & 75 & 1831 & 48 & -3.44 \\
\hline 74 & 20 & 7,886 & 4.055 & 0.254 & 0.2842 & 0.0150 & 0.1035 & 0.0027 & 1645 & 51 & 1613 & 75 & 1687 & 49 & -1.05 \\
\hline 75 & 20 & 11,938 & 5.637 & 0.401 & 0.3954 & 0.0240 & 0.1034 & 0.0027 & 1922 & 61 & 2148 & 111 & 1686 & 49 & 3.10 \\
\hline 76 & 19 & 20,914 & 4.466 & 0.294 & 0.3097 & 0.0175 & 0.1046 & 0.0027 & 1725 & 55 & 1739 & 86 & 1707 & 48 & 0.38 \\
\hline 77 & 19 & 14,488 & 4.433 & 0.288 & 0.3083 & 0.0171 & 0.1043 & 0.0027 & 1719 & 54 & 1732 & 84 & 1702 & 48 & 0.36 \\
\hline 78 & 19 & 18,310 & 3.874 & 0.240 & 0.2690 & 0.0141 & 0.1045 & 0.0027 & 1608 & 50 & 1536 & 72 & 1705 & 48 & -2.67 \\
\hline 79 & 16 & 18,585 & 3.942 & 0.249 & 0.2747 & 0.0147 & 0.1041 & 0.0028 & 1622 & 51 & 1565 & 74 & 1698 & 49 & -2.01 \\
\hline 80 & 15 & 13,070 & 4.248 & 0.276 & 0.2870 & 0.0158 & 0.1073 & 0.0029 & 1683 & 53 & 1627 & 79 & 1755 & 50 & -1.80 \\
\hline 81 & 18 & 14,649 & 3.836 & 0.239 & 0.2673 & 0.0141 & 0.1041 & 0.0028 & 1600 & 50 & 1527 & 72 & 1698 & 49 & -2.72 \\
\hline 82 & 19 & 26,175 & 5.017 & 0.327 & 0.3248 & 0.0180 & 0.1120 & 0.0029 & 1822 & 55 & 1813 & 88 & 1833 & 47 & -0.21 \\
\hline 83 & 20 & 13,040 & 4.008 & 0.249 & 0.2779 & 0.0146 & 0.1046 & 0.0027 & 1636 & 51 & 1581 & 74 & 1707 & 48 & -1.86 \\
\hline 84 & 13 & 17,376 & 4.100 & 0.269 & 0.2863 & 0.0158 & 0.1039 & 0.0029 & 1654 & 54 & 1623 & 79 & 1694 & 52 & -0.99 \\
\hline 85 & 10 & 21,779 & 4.138 & 0.285 & 0.2866 & 0.0168 & 0.1047 & 0.0030 & 1662 & 56 & 1624 & 84 & 1709 & 52 & -1.18 \\
\hline 86 & 17 & 9,196 & 4.900 & 0.331 & 0.3436 & 0.0196 & 0.1034 & 0.0028 & 1802 & 57 & 1904 & 94 & 1686 & 50 & 2.03 \\
\hline 87 & 19 & 24,137 & 5.937 & 0.422 & 0.3813 & 0.0232 & 0.1129 & 0.0030 & 1967 & 62 & 2082 & 108 & 1847 & 48 & 1.82 \\
\hline 88 & 18 & 19,282 & 4.553 & 0.297 & 0.3173 & 0.0176 & 0.1041 & 0.0027 & 1741 & 54 & 1776 & 86 & 1698 & 48 & 0.87 \\
\hline
\end{tabular}


Isotopic ratios (corrected)

Ages (Ma)

\begin{tabular}{|c|c|c|c|c|c|c|c|c|c|c|c|c|c|c|c|}
\hline \# & $\mathrm{N}$ & $\frac{{ }^{206} \mathrm{~Pb}}{{ }^{204} \mathrm{~Pb}^{\dagger}}$ & $\begin{array}{l}{ }^{2077} \mathrm{~Pb}^{*} \\
{ }^{235} \mathrm{U}\end{array}$ & $\begin{array}{c} \pm \\
(\mathrm{abs})\end{array}$ & $\begin{array}{l}{ }^{206} \mathrm{~Pb}^{*} \\
{ }^{238} \mathrm{U}\end{array}$ & $\begin{array}{c} \pm \\
\text { (abs) }\end{array}$ & $\begin{array}{l}{ }^{207} \mathrm{~Pb}^{*} \\
{ }^{206} \mathrm{~Pb}^{*}\end{array}$ & $\begin{array}{c} \pm \\
\text { (abs) }\end{array}$ & $\begin{array}{c}{ }^{207} \mathrm{~Pb}^{*} \\
{ }^{235} \mathrm{U}\end{array}$ & $\begin{array}{c} \pm \pm \\
\text { (abs) }\end{array}$ & $\begin{array}{c}{ }^{206} \mathrm{~Pb}^{*} \\
{ }^{238} \mathrm{U}\end{array}$ & $\begin{array}{c} \pm \\
(\mathrm{abs})\end{array}$ & $\begin{array}{l}{ }^{207} \mathrm{~Pb}^{*} \\
{ }^{206} \mathrm{~Pb}^{*}\end{array}$ & $\begin{array}{c} \pm \\
(\mathrm{abs})\end{array}$ & $\begin{array}{c}\text { Disc. } \\
\text { (\%) }\end{array}$ \\
\hline 89 & 20 & 28,295 & 9.812 & 0.709 & 0.4232 & 0.0261 & 0.1682 & 0.0044 & 2417 & 67 & 2275 & 118 & 2539 & 44 & -2.00 \\
\hline 90 & 19 & 24,023 & 6.411 & 0.459 & 0.4068 & 0.0248 & 0.1143 & 0.0030 & 2034 & 63 & 2200 & 114 & 1869 & 47 & 2.20 \\
\hline 91 & 19 & 19,565 & 5.615 & 0.413 & 0.3929 & 0.0250 & 0.1037 & 0.0027 & 1918 & 63 & 2136 & 116 & 1691 & 49 & 3.04 \\
\hline 92 & 18 & 30,830 & 5.672 & 0.392 & 0.3759 & 0.0221 & 0.1094 & 0.0029 & 1927 & 60 & 2057 & 104 & 1790 & 48 & 2.09 \\
\hline 93 & 19 & 10,926 & 4.111 & 0.260 & 0.2864 & 0.0153 & 0.1041 & 0.0028 & 1657 & 52 & 1623 & 77 & 1699 & 49 & -1.04 \\
\hline 94 & 16 & 9,845 & 4.056 & 0.258 & 0.2827 & 0.0152 & 0.1040 & 0.0028 & 1645 & 52 & 1605 & 76 & 1698 & 50 & -1.32 \\
\hline 95 & 18 & 16,273 & 4.109 & 0.259 & 0.2855 & 0.0152 & 0.1044 & 0.0028 & 1656 & 51 & 1619 & 76 & 1703 & 49 & -1.18 \\
\hline 96 & 20 & 19,730 & 3.905 & 0.242 & 0.2709 & 0.0142 & 0.1045 & 0.0027 & 1615 & 50 & 1546 & 72 & 1706 & 48 & -2.50 \\
\hline 97 & 18 & 18,725 & 4.602 & 0.313 & 0.3211 & 0.0188 & 0.1039 & 0.0027 & 1750 & 57 & 1795 & 92 & 1696 & 48 & 1.08 \\
\hline 98 & 20 & 22,274 & 5.460 & 0.397 & 0.3890 & 0.0245 & 0.1018 & 0.0027 & 1894 & 62 & 2118 & 114 & 1657 & 48 & 3.19 \\
\hline 99 & 19 & 15,101 & 5.091 & 0.341 & 0.3480 & 0.0198 & 0.1061 & 0.0028 & 1835 & 57 & 1925 & 95 & 1734 & 48 & 1.76 \\
\hline 100 & 17 & 14,933 & 3.994 & 0.252 & 0.2800 & 0.0150 & 0.1035 & 0.0028 & 1633 & 51 & 1591 & 75 & 1687 & 49 & -1.39 \\
\hline 101 & 18 & 18,194 & 4.826 & 0.309 & 0.3098 & 0.0168 & 0.1130 & 0.0030 & 1789 & 54 & 1740 & 83 & 1848 & 48 & -1.34 \\
\hline
\end{tabular}

\begin{tabular}{|c|c|c|c|c|c|c|c|c|c|c|c|c|c|c|c|}
\hline \multicolumn{16}{|c|}{ Sample ORT-N: Quartzite, Ortega Formation, Tusas Mountains, New Mexico } \\
\hline 1 & 19 & 30,746 & 3.984 & 0.293 & 0.2730 & 0.0173 & 0.1059 & 0.0028 & 1631 & 60 & 1556 & 88 & 1729 & 48 & 2.68 \\
\hline 2 & 12 & 2,500 & 3.061 & 0.277 & 0.2114 & 0.0164 & 0.1050 & 0.0031 & 1423 & 69 & 1236 & 87 & 1715 & 54 & 11.92 \\
\hline 3 & 11 & 4,054 & 3.384 & 0.532 & 0.2306 & 0.0341 & 0.1064 & 0.0031 & 1501 & 123 & 1338 & 179 & 1739 & 53 & 8.60 \\
\hline 4 & 13 & 6,790 & 3.850 & 0.294 & 0.2485 & 0.0161 & 0.1124 & 0.0031 & 1603 & 62 & 1431 & 83 & 1838 & 51 & 7.85 \\
\hline 5 & 20 & 5,697 & 7.111 & 0.567 & 0.2828 & 0.0197 & 0.1824 & 0.0048 & 2125 & 71 & 1605 & 99 & 2675 & 44 & 21.67 \\
\hline 6 & 17 & 5,615 & 3.776 & 0.283 & 0.2523 & 0.0161 & 0.1085 & 0.0029 & 1588 & 60 & 1450 & 83 & 1775 & 49 & 5.94 \\
\hline 7 & 15 & 4,693 & 4.508 & 0.365 & 0.3012 & 0.0207 & 0.1086 & 0.0030 & 1732 & 67 & 1697 & 102 & 1776 & 51 & 1.00 \\
\hline 8 & 11 & 2,669 & 4.092 & 0.370 & 0.2806 & 0.0213 & 0.1058 & 0.0032 & 1653 & 74 & 1594 & 107 & 1728 & 56 & 1.95 \\
\hline 9 & 19 & 3,739 & 4.347 & 0.616 & 0.2986 & 0.0394 & 0.1056 & 0.0029 & 1702 & 117 & 1684 & 195 & 1724 & 50 & 0.51 \\
\hline 10 & 20 & 23,635 & 5.375 & 0.424 & 0.3685 & 0.0249 & 0.1058 & 0.0028 & 1881 & 68 & 2022 & 117 & 1728 & 48 & -2.37 \\
\hline 11 & 18 & 6,958 & 4.252 & 0.309 & 0.2930 & 0.0179 & 0.1052 & 0.0028 & 1684 & 60 & 1656 & 89 & 1719 & 49 & 0.83 \\
\hline 12 & 20 & 10,216 & 3.717 & 0.272 & 0.2560 & 0.0160 & 0.1053 & 0.0028 & 1575 & 58 & 1469 & 82 & 1720 & 49 & 4.38 \\
\hline 13 & 11 & 4,970 & 3.723 & 0.284 & 0.2511 & 0.0160 & 0.1075 & 0.0031 & 1576 & 61 & 1444 & 83 & 1758 & 53 & 5.76 \\
\hline 14 & 16 & 23,301 & 4.318 & 0.356 & 0.2869 & 0.0209 & 0.1092 & 0.0029 & 1697 & 68 & 1626 & 105 & 1785 & 49 & 2.26 \\
\hline 15 & 15 & 5,790 & 4.560 & 0.363 & 0.3002 & 0.0203 & 0.1102 & 0.0030 & 1742 & 66 & 1692 & 101 & 1802 & 50 & 1.43 \\
\hline 16 & 20 & 7,989 & 5.065 & 0.405 & 0.3486 & 0.0238 & 0.1054 & 0.0028 & 1830 & 68 & 1928 & 114 & 1721 & 49 & -1.89 \\
\hline 17 & 19 & 10,510 & 5.081 & 0.420 & 0.3497 & 0.0250 & 0.1054 & 0.0028 & 1833 & 70 & 1933 & 119 & 1721 & 49 & -1.92 \\
\hline 18 & 17 & 1,855 & 4.115 & 0.331 & 0.2812 & 0.0184 & 0.1061 & 0.0030 & 1657 & 66 & 1598 & 92 & 1734 & 52 & 1.98 \\
\hline 19 & 18 & 15,982 & 3.826 & 0.266 & 0.2625 & 0.0155 & 0.1057 & 0.0028 & 1598 & 56 & 1503 & 79 & 1727 & 48 & 3.74 \\
\hline 20 & 14 & 7,631 & 5.254 & 0.597 & 0.3456 & 0.0361 & 0.1103 & 0.0030 & 1861 & 97 & 1914 & 173 & 1804 & 50 & -1.05 \\
\hline 21 & 19 & 13,921 & 4.380 & 0.322 & 0.3007 & 0.0188 & 0.1056 & 0.0028 & 1709 & 61 & 1695 & 93 & 1726 & 48 & 0.39 \\
\hline 22 & 19 & 7,620 & 4.923 & 0.414 & 0.3361 & 0.0245 & 0.1062 & 0.0028 & 1806 & 71 & 1868 & 118 & 1736 & 49 & -1.31 \\
\hline 23 & 13 & 4,943 & 3.635 & 0.267 & 0.2461 & 0.0149 & 0.1071 & 0.0031 & 1557 & 58 & 1418 & 77 & 1751 & 52 & 6.34 \\
\hline 24 & 16 & 4,923 & 4.316 & 0.329 & 0.2960 & 0.0189 & 0.1058 & 0.0029 & 1696 & 63 & 1671 & 94 & 1727 & 50 & 0.74 \\
\hline 25 & 12 & 5,414 & 3.455 & 0.356 & 0.2291 & 0.0215 & 0.1094 & 0.0031 & 1517 & 81 & 1330 & 113 & 1789 & 51 & 10.15 \\
\hline 26 & 19 & 14,412 & 4.489 & 0.335 & 0.3088 & 0.0197 & 0.1054 & 0.0028 & 1729 & 62 & 1735 & 97 & 1722 & 49 & -0.15 \\
\hline 27 & 11 & 4,963 & 3.787 & 0.295 & 0.2653 & 0.0171 & 0.1035 & 0.0031 & 1590 & 63 & 1517 & 87 & 1688 & 55 & 2.76 \\
\hline 28 & 15 & 7,093 & 3.713 & 0.273 & 0.2568 & 0.0159 & 0.1049 & 0.0029 & 1574 & 59 & 1473 & 82 & 1712 & 50 & 4.14 \\
\hline 29 & 19 & 23,357 & 4.067 & 0.286 & 0.2781 & 0.0166 & 0.1061 & 0.0028 & 1648 & 57 & 1582 & 84 & 1733 & 48 & 2.24 \\
\hline 30 & 20 & 11,852 & 3.959 & 0.281 & 0.2735 & 0.0164 & 0.1050 & 0.0028 & 1626 & 58 & 1559 & 83 & 1714 & 49 & 2.38 \\
\hline 31 & 19 & 11,140 & 3.656 & 0.251 & 0.2453 & 0.0142 & 0.1081 & 0.0029 & 1562 & 55 & 1414 & 73 & 1767 & 48 & 6.81 \\
\hline
\end{tabular}


Isotopic ratios (corrected)

Ages (Ma)

\begin{tabular}{|c|c|c|c|c|c|c|c|c|c|c|c|c|c|c|c|}
\hline$\#$ & $\mathrm{~N}$ & $\frac{{ }^{206} \mathrm{~Pb}}{{ }^{204} \mathrm{~Pb}^{\dagger}}$ & $\begin{array}{c}{ }^{207} \mathrm{~Pb}^{*} \\
{ }^{235} \mathrm{U}\end{array}$ & $\begin{array}{c} \pm \\
\text { (abs) }\end{array}$ & $\begin{array}{c}{ }^{206} \mathrm{~Pb}^{*} \\
{ }^{238} \mathrm{U}\end{array}$ & $\begin{array}{c} \pm \\
(\mathrm{abs})\end{array}$ & $\begin{array}{l}{ }^{207} \mathrm{~Pb}^{*} \\
{ }^{206} \mathrm{~Pb}^{*}\end{array}$ & $\begin{array}{c} \pm \\
\text { (abs) }\end{array}$ & $\begin{array}{c}{ }^{207} \mathrm{~Pb}^{*} \\
{ }^{235} \mathrm{U}\end{array}$ & $\begin{array}{c} \pm \\
\text { (abs) }\end{array}$ & $\begin{array}{c}{ }^{206} \mathrm{~Pb}^{*} \\
{ }^{238} \mathrm{U}\end{array}$ & $\begin{array}{c} \pm \\
(\mathrm{abs})\end{array}$ & $\begin{array}{l}{ }^{207} \mathrm{~Pb}^{*} \\
{ }^{206} \mathrm{~Pb}^{*}\end{array}$ & $\begin{array}{c} \pm \\
(\mathrm{abs})\end{array}$ & $\begin{array}{c}\text { Disc. } \\
(\%)\end{array}$ \\
\hline 32 & 20 & 6,903 & 4.018 & 0.310 & 0.2761 & 0.0183 & 0.1056 & 0.0028 & 1638 & 63 & 1571 & 93 & 1724 & 49 & 2.30 \\
\hline 33 & 19 & 12,941 & 3.940 & 0.317 & 0.2694 & 0.0191 & 0.1061 & 0.0028 & 1622 & 65 & 1538 & 97 & 1733 & 48 & 3.10 \\
\hline 34 & 12 & 1,896 & 3.986 & 0.388 & 0.2737 & 0.0226 & 0.1056 & 0.0031 & 1631 & 79 & 1560 & 114 & 1725 & 54 & 2.53 \\
\hline 35 & 15 & 2,171 & 3.818 & 0.292 & 0.2620 & 0.0162 & 0.1057 & 0.0030 & 1597 & 62 & 1500 & 83 & 1726 & 52 & 3.78 \\
\hline 36 & 16 & 10,403 & 3.579 & 0.248 & 0.2400 & 0.0140 & 0.1081 & 0.0029 & 1545 & 55 & 1387 & 73 & 1768 & 49 & 7.67 \\
\hline 37 & 20 & 14,217 & 4.637 & 0.370 & 0.3194 & 0.0222 & 0.1053 & 0.0028 & 1756 & 67 & 1787 & 108 & 1719 & 48 & -0.75 \\
\hline 38 & 19 & 19,135 & 5.232 & 0.411 & 0.3599 & 0.0241 & 0.1054 & 0.0028 & 1858 & 67 & 1982 & 114 & 1722 & 48 & -2.20 \\
\hline 39 & 19 & 11,995 & 3.596 & 0.250 & 0.2434 & 0.0144 & 0.1071 & 0.0028 & 1549 & 55 & 1404 & 75 & 1751 & 48 & 6.76 \\
\hline 40 & 12 & 1,566 & 4.707 & 0.469 & 0.3220 & 0.0266 & 0.1060 & 0.0032 & 1768 & 83 & 1799 & 130 & 1732 & 55 & -0.74 \\
\hline 41 & 15 & 5,686 & 4.995 & 0.533 & 0.3464 & 0.0333 & 0.1046 & 0.0029 & 1818 & 90 & 1917 & 159 & 1707 & 51 & -1.94 \\
\hline 42 & 18 & 7,737 & 4.641 & 0.407 & 0.3201 & 0.0247 & 0.1052 & 0.0028 & 1757 & 73 & 1790 & 121 & 1717 & 49 & -0.81 \\
\hline 43 & 19 & 8,601 & 4.030 & 0.292 & 0.2779 & 0.0170 & 0.1052 & 0.0028 & 1640 & 59 & 1581 & 86 & 1718 & 49 & 2.03 \\
\hline 44 & 20 & 17,277 & 4.503 & 0.355 & 0.3000 & 0.0207 & 0.1089 & 0.0029 & 1731 & 66 & 1691 & 103 & 1780 & 48 & 1.15 \\
\hline 45 & 11 & 835 & 3.959 & 0.387 & 0.2668 & 0.0199 & 0.1076 & 0.0036 & 1626 & 79 & 1524 & 101 & 1760 & 60 & 3.84 \\
\hline 46 & 16 & 9,157 & 5.029 & 0.517 & 0.3465 & 0.0323 & 0.1053 & 0.0028 & 1824 & 87 & 1918 & 155 & 1719 & 49 & -1.84 \\
\hline 47 & 19 & 31,766 & 4.693 & 0.391 & 0.3223 & 0.0238 & 0.1056 & 0.0028 & 1766 & 70 & 1801 & 116 & 1725 & 48 & -0.83 \\
\hline 48 & 19 & 9,151 & 3.664 & 0.260 & 0.2554 & 0.0153 & 0.1041 & 0.0028 & 1564 & 57 & 1466 & 79 & 1698 & 49 & 4.04 \\
\hline 49 & 16 & 9,581 & 4.789 & 0.484 & 0.3309 & 0.0303 & 0.1050 & 0.0028 & 1783 & 85 & 1843 & 147 & 1714 & 50 & -1.31 \\
\hline 50 & 20 & 6,230 & 5.299 & 0.446 & 0.3649 & 0.0262 & 0.1053 & 0.0028 & 1869 & 72 & 2006 & 124 & 1720 & 49 & -2.35 \\
\hline 51 & 19 & 9,382 & 5.090 & 0.403 & 0.3517 & 0.0237 & 0.1050 & 0.0028 & 1834 & 67 & 1942 & 113 & 1714 & 49 & -2.04 \\
\hline 52 & 16 & 1,822 & 4.079 & 0.328 & 0.2796 & 0.0181 & 0.1058 & 0.0030 & 1650 & 65 & 1589 & 91 & 1728 & 53 & 2.04 \\
\hline 53 & 13 & 5,545 & 4.363 & 0.733 & 0.3015 & 0.0481 & 0.1049 & 0.0029 & 1705 & 139 & 1699 & 238 & 1713 & 51 & 0.18 \\
\hline 54 & 19 & 13,873 & 4.025 & 0.293 & 0.2771 & 0.0172 & 0.1053 & 0.0028 & 1639 & 59 & 1577 & 87 & 1720 & 48 & 2.15 \\
\hline 55 & 19 & 9,619 & 3.905 & 0.288 & 0.2695 & 0.0170 & 0.1051 & 0.0028 & 1615 & 60 & 1538 & 86 & 1716 & 49 & 2.81 \\
\hline 56 & 16 & 7,772 & 3.890 & 0.285 & 0.2700 & 0.0167 & 0.1045 & 0.0028 & 1612 & 59 & 1541 & 85 & 1705 & 50 & 2.56 \\
\hline 57 & 15 & 9,880 & 3.829 & 0.276 & 0.2657 & 0.0161 & 0.1045 & 0.0029 & 1599 & 58 & 1519 & 82 & 1706 & 50 & 3.02 \\
\hline 58 & 17 & 2,406 & 3.580 & 0.291 & 0.2443 & 0.0161 & 0.1063 & 0.0033 & 1545 & 64 & 1409 & 83 & 1737 & 57 & 6.31 \\
\hline 59 & 12 & 22,042 & 10.271 & 1.570 & 0.3853 & 0.0562 & 0.1934 & 0.0055 & 2460 & 141 & 2101 & 261 & 2771 & 47 & 6.98 \\
\hline 60 & 19 & 12,201 & 3.937 & 0.282 & 0.2602 & 0.0159 & 0.1097 & 0.0029 & 1621 & 58 & 1491 & 82 & 1795 & 48 & 5.28 \\
\hline 61 & 15 & 9,498 & 3.965 & 0.314 & 0.2737 & 0.0188 & 0.1051 & 0.0028 & 1627 & 64 & 1560 & 95 & 1716 & 50 & 2.38 \\
\hline 62 & 12 & 3,655 & 3.157 & 0.255 & 0.2097 & 0.0145 & 0.1092 & 0.0031 & 1447 & 62 & 1227 & 77 & 1785 & 52 & 14.47 \\
\hline 63 & 15 & 5,398 & 5.136 & 0.409 & 0.3506 & 0.0233 & 0.1062 & 0.0029 & 1842 & 68 & 1937 & 111 & 1736 & 50 & -1.82 \\
\hline 64 & 15 & 1,890 & 4.552 & 0.397 & 0.3112 & 0.0222 & 0.1061 & 0.0031 & 1740 & 73 & 1747 & 109 & 1733 & 53 & -0.16 \\
\hline 65 & 18 & 12,634 & 5.926 & 0.492 & 0.3961 & 0.0280 & 0.1085 & 0.0029 & 1965 & 72 & 2151 & 129 & 1775 & 48 & -2.59 \\
\hline 66 & 15 & 2,795 & 5.742 & 0.567 & 0.3574 & 0.0304 & 0.1165 & 0.0033 & 1938 & 85 & 1970 & 145 & 1904 & 51 & -0.61 \\
\hline 67 & 19 & 16,659 & 5.463 & 0.440 & 0.3772 & 0.0259 & 0.1050 & 0.0028 & 1895 & 69 & 2063 & 121 & 1715 & 48 & -2.64 \\
\hline 68 & 14 & 4,337 & 5.498 & 0.497 & 0.3779 & 0.0290 & 0.1055 & 0.0030 & 1900 & 78 & 2067 & 136 & 1723 & 52 & -2.60 \\
\hline 69 & 13 & 1,874 & 5.122 & 0.597 & 0.3585 & 0.0357 & 0.1036 & 0.0032 & 1840 & 99 & 1975 & 169 & 1690 & 57 & -2.41 \\
\hline 70 & 20 & 9,048 & 4.879 & 0.379 & 0.3360 & 0.0222 & 0.1053 & 0.0028 & 1799 & 66 & 1867 & 107 & 1720 & 49 & -1.46 \\
\hline 71 & 9 & 525 & 5.198 & 0.722 & 0.3320 & 0.0345 & 0.1136 & 0.0042 & 1852 & 118 & 1848 & 167 & 1857 & 66 & 0.10 \\
\hline 72 & 20 & 14,257 & 3.821 & 0.268 & 0.2554 & 0.0153 & 0.1085 & 0.0028 & 1597 & 57 & 1466 & 78 & 1775 & 48 & 5.51 \\
\hline 73 & 13 & 2,926 & 3.541 & 0.453 & 0.2440 & 0.0285 & 0.1052 & 0.0030 & 1536 & 101 & 1408 & 147 & 1719 & 53 & 5.96 \\
\hline 74 & 14 & 3,813 & 4.581 & 0.366 & 0.3154 & 0.0210 & 0.1054 & 0.0029 & 1746 & 67 & 1767 & 103 & 1721 & 51 & -0.53 \\
\hline 75 & 12 & 6,918 & 5.236 & 0.462 & 0.3625 & 0.0274 & 0.1048 & 0.0029 & 1859 & 75 & 1994 & 130 & 1710 & 51 & -2.36 \\
\hline 76 & 13 & 5,790 & 3.668 & 0.372 & 0.2511 & 0.0230 & 0.1059 & 0.0030 & 1564 & 81 & 1444 & 119 & 1730 & 52 & 5.21 \\
\hline 77 & 19 & 14,196 & 3.557 & 0.241 & 0.2375 & 0.0135 & 0.1086 & 0.0029 & 1540 & 54 & 1374 & 70 & 1776 & 49 & 8.28 \\
\hline
\end{tabular}


Isotopic ratios (corrected)

Ages (Ma)

\begin{tabular}{|c|c|c|c|c|c|c|c|c|c|c|c|c|c|c|c|}
\hline$\#$ & $\mathrm{~N}$ & $\frac{{ }^{206} \mathrm{~Pb}}{{ }^{204} \mathrm{~Pb}^{\dagger}}$ & $\begin{array}{c}{ }^{207} \mathrm{~Pb}^{*} \\
{ }^{235} \mathrm{U}\end{array}$ & $\begin{array}{c} \pm \\
(\mathrm{abs})\end{array}$ & $\begin{array}{l}{ }^{206} \mathrm{~Pb}^{*} \\
{ }^{238} \mathrm{U}\end{array}$ & $\begin{array}{c} \pm \\
\text { (abs) }\end{array}$ & $\begin{array}{l}{ }^{207} \mathrm{~Pb}^{*} \\
{ }^{206} \mathrm{~Pb}^{*}\end{array}$ & $\begin{array}{c} \pm \\
(\mathrm{abs})\end{array}$ & $\begin{array}{c}{ }^{207} \mathrm{~Pb}^{*} \\
{ }^{235} \mathrm{U}\end{array}$ & $\begin{array}{c} \pm \\
(\mathrm{abs})\end{array}$ & $\begin{array}{c}{ }^{206} \mathrm{~Pb}^{*} \\
{ }^{238} \mathrm{U}\end{array}$ & $\begin{array}{c} \pm \\
(\mathrm{abs})\end{array}$ & $\begin{array}{l}{ }^{207} \mathrm{~Pb}^{*} \\
{ }^{206} \mathrm{~Pb}^{*}\end{array}$ & $\begin{array}{c} \pm \\
(\mathrm{abs})\end{array}$ & $\begin{array}{c}\text { Disc. } \\
(\%)\end{array}$ \\
\hline 78 & 9 & 14,340 & 3.789 & 0.307 & 0.2558 & 0.0178 & 0.1074 & 0.0031 & 1590 & 65 & 1468 & 92 & 1756 & 54 & 5.10 \\
\hline 79 & 19 & 20,325 & 3.533 & 0.260 & 0.2256 & 0.0144 & 0.1136 & 0.0030 & 1535 & 58 & 1311 & 76 & 1857 & 48 & 12.73 \\
\hline 80 & 19 & 4,794 & 3.872 & 0.292 & 0.2666 & 0.0169 & 0.1054 & 0.0029 & 1608 & 61 & 1523 & 86 & 1720 & 50 & 3.18 \\
\hline 81 & 11 & 3,845 & 3.979 & 0.486 & 0.2752 & 0.0299 & 0.1049 & 0.0036 & 1630 & 99 & 1567 & 151 & 1712 & 63 & 2.19 \\
\hline 82 & 19 & 16,498 & 5.270 & 0.406 & 0.3371 & 0.0222 & 0.1134 & 0.0030 & 1864 & 66 & 1873 & 107 & 1854 & 48 & -0.19 \\
\hline 83 & 17 & 9,361 & 3.630 & 0.256 & 0.2511 & 0.0149 & 0.1049 & 0.0028 & 1556 & 56 & 1444 & 77 & 1712 & 49 & 4.85 \\
\hline 84 & 20 & 3,424 & 4.490 & 0.397 & 0.3101 & 0.0236 & 0.1050 & 0.0029 & 1729 & 73 & 1741 & 116 & 1715 & 50 & -0.31 \\
\hline 85 & 13 & 5,713 & 3.812 & 0.302 & 0.2634 & 0.0178 & 0.1049 & 0.0029 & 1595 & 64 & 1507 & 91 & 1713 & 52 & 3.40 \\
\hline 86 & 13 & 7,049 & 4.240 & 0.353 & 0.2952 & 0.0212 & 0.1042 & 0.0029 & 1682 & 68 & 1667 & 105 & 1700 & 52 & 0.43 \\
\hline 87 & 11 & 6,837 & 3.918 & 0.369 & 0.2730 & 0.0228 & 0.1041 & 0.0030 & 1617 & 76 & 1556 & 115 & 1698 & 53 & 2.16 \\
\hline 88 & 7 & 1,782 & 4.451 & 0.540 & 0.2998 & 0.0294 & 0.1077 & 0.0045 & 1722 & 101 & 1691 & 146 & 1760 & 77 & 0.89 \\
\hline 89 & 13 & 896 & 4.040 & 0.479 & 0.2631 & 0.0254 & 0.1113 & 0.0036 & 1642 & 96 & 1506 & 130 & 1822 & 58 & 5.42 \\
\hline 90 & 19 & 4,486 & 5.153 & 0.414 & 0.3577 & 0.0239 & 0.1045 & 0.0029 & 1845 & 68 & 1971 & 113 & 1705 & 50 & -2.28 \\
\hline 91 & 14 & 12,398 & 4.086 & 0.324 & 0.2819 & 0.0194 & 0.1051 & 0.0029 & 1652 & 65 & 1601 & 97 & 1717 & 50 & 1.67 \\
\hline 92 & 20 & 14,992 & 4.839 & 0.439 & 0.3321 & 0.0270 & 0.1057 & 0.0028 & 1792 & 76 & 1849 & 131 & 1726 & 48 & -1.25 \\
\hline 93 & 15 & 8,439 & 3.844 & 0.295 & 0.2541 & 0.0169 & 0.1097 & 0.0030 & 1602 & 62 & 1459 & 87 & 1795 & 49 & 6.11 \\
\hline 94 & 20 & 12,631 & 3.584 & 0.253 & 0.2466 & 0.0149 & 0.1054 & 0.0028 & 1546 & 56 & 1421 & 77 & 1721 & 48 & 5.64 \\
\hline 95 & 8 & 2,509 & 4.024 & 0.441 & 0.2757 & 0.0254 & 0.1059 & 0.0039 & 1639 & 89 & 1570 & 128 & 1729 & 67 & 2.42 \\
\hline 96 & 20 & 11,559 & 4.506 & 0.347 & 0.3098 & 0.0204 & 0.1055 & 0.0028 & 1732 & 64 & 1740 & 101 & 1723 & 49 & -0.20 \\
\hline 97 & 10 & 1,994 & 5.364 & 0.521 & 0.3634 & 0.0285 & 0.1071 & 0.0034 & 1879 & 83 & 1998 & 135 & 1750 & 59 & -2.08 \\
\hline 98 & 14 & 3,260 & 4.716 & 0.476 & 0.3114 & 0.0277 & 0.1098 & 0.0031 & 1770 & 85 & 1748 & 136 & 1797 & 52 & 0.59 \\
\hline
\end{tabular}

\begin{tabular}{|c|c|c|c|c|c|c|c|c|c|c|c|c|c|c|c|}
\hline \multicolumn{16}{|c|}{ Sample LC-CC-15: Quartzite, Cebolla Canyon, south of Gunnison, Colorado } \\
\hline 1 & 18 & 18,165 & 3.910 & 0.231 & 0.2635 & 0.0131 & 0.1076 & 0.0029 & 1616 & 48 & 1508 & 67 & 1760 & 49 & 4.21 \\
\hline 2 & 15 & 12,150 & 4.355 & 0.279 & 0.2902 & 0.0158 & 0.1088 & 0.0030 & 1704 & 53 & 1643 & 79 & 1780 & 50 & 1.90 \\
\hline 3 & 20 & 14,373 & 3.980 & 0.236 & 0.2699 & 0.0135 & 0.1070 & 0.0028 & 1630 & 48 & 1540 & 69 & 1748 & 48 & 3.31 \\
\hline 4 & 19 & 11,239 & 4.613 & 0.316 & 0.3153 & 0.0189 & 0.1061 & 0.0028 & 1752 & 57 & 1767 & 92 & 1734 & 48 & -0.38 \\
\hline 5 & 18 & 13,073 & 4.364 & 0.287 & 0.2966 & 0.0169 & 0.1067 & 0.0028 & 1706 & 54 & 1675 & 84 & 1744 & 48 & 0.90 \\
\hline 6 & 16 & 12,021 & 4.198 & 0.301 & 0.2837 & 0.0180 & 0.1073 & 0.0029 & 1674 & 59 & 1610 & 91 & 1754 & 49 & 2.07 \\
\hline 7 & 11 & 5,881 & 3.439 & 0.282 & 0.2352 & 0.0172 & 0.1060 & 0.0031 & 1513 & 65 & 1362 & 90 & 1732 & 53 & 7.63 \\
\hline 8 & 18 & 11,323 & 3.707 & 0.219 & 0.2523 & 0.0125 & 0.1066 & 0.0028 & 1573 & 47 & 1451 & 64 & 1741 & 49 & 5.27 \\
\hline 9 & 20 & 23,333 & 3.659 & 0.213 & 0.2471 & 0.0122 & 0.1074 & 0.0028 & 1562 & 46 & 1424 & 63 & 1755 & 48 & 6.28 \\
\hline 10 & 20 & 23,675 & 3.820 & 0.225 & 0.2585 & 0.0129 & 0.1072 & 0.0028 & 1597 & 47 & 1482 & 66 & 1752 & 48 & 4.68 \\
\hline 11 & 20 & 4,907 & 3.002 & 0.425 & 0.2024 & 0.0273 & 0.1076 & 0.0029 & 1408 & 108 & 1188 & 146 & 1758 & 49 & 15.58 \\
\hline 12 & 20 & 13,155 & 3.711 & 0.218 & 0.2523 & 0.0124 & 0.1067 & 0.0029 & 1574 & 47 & 1450 & 64 & 1744 & 49 & 5.32 \\
\hline 13 & 18 & 13,856 & 4.080 & 0.256 & 0.2791 & 0.0150 & 0.1060 & 0.0028 & 1650 & 51 & 1587 & 76 & 1732 & 49 & 2.15 \\
\hline 14 & 17 & 12,076 & 4.071 & 0.246 & 0.2700 & 0.0138 & 0.1094 & 0.0029 & 1649 & 49 & 1541 & 70 & 1789 & 49 & 4.00 \\
\hline 15 & 17 & 8,361 & 3.856 & 0.232 & 0.2523 & 0.0128 & 0.1108 & 0.0030 & 1604 & 49 & 1450 & 66 & 1813 & 49 & 6.74 \\
\hline 16 & 20 & 22,590 & 3.778 & 0.219 & 0.2515 & 0.0124 & 0.1089 & 0.0028 & 1588 & 47 & 1446 & 64 & 1782 & 48 & 6.19 \\
\hline 17 & 19 & 24,859 & 3.936 & 0.232 & 0.2615 & 0.0130 & 0.1092 & 0.0029 & 1621 & 48 & 1497 & 67 & 1786 & 48 & 4.94 \\
\hline 18 & 15 & 9,364 & 3.800 & 0.231 & 0.2587 & 0.0132 & 0.1065 & 0.0029 & 1593 & 49 & 1483 & 67 & 1741 & 50 & 4.44 \\
\hline 19 & 19 & 33,590 & 10.235 & 0.661 & 0.3865 & 0.0212 & 0.1921 & 0.0050 & 2456 & 60 & 2107 & 99 & 2760 & 43 & 6.72 \\
\hline 20 & 19 & 16,146 & 3.545 & 0.208 & 0.2428 & 0.0120 & 0.1059 & 0.0028 & 1537 & 46 & 1401 & 62 & 1730 & 48 & 6.38 \\
\hline 21 & 17 & 27,084 & 3.942 & 0.233 & 0.2607 & 0.0131 & 0.1096 & 0.0029 & 1622 & 48 & 1494 & 67 & 1793 & 48 & 5.18 \\
\hline 22 & 19 & 11,682 & 3.866 & 0.228 & 0.2618 & 0.0130 & 0.1071 & 0.0028 & 1607 & 48 & 1499 & 66 & 1750 & 49 & 4.25 \\
\hline 23 & 19 & 5,756 & 3.518 & 0.210 & 0.2425 & 0.0121 & 0.1052 & 0.0028 & 1531 & 47 & 1400 & 63 & 1718 & 50 & 6.17 \\
\hline
\end{tabular}


Isotopic ratios (corrected)

Ages (Ma)

\begin{tabular}{|c|c|c|c|c|c|c|c|c|c|c|c|c|c|c|c|}
\hline$\#$ & $\mathrm{~N}$ & $\frac{{ }^{206} \mathrm{~Pb}}{{ }^{204} \mathrm{~Pb}^{\dagger}}$ & $\begin{array}{l}{ }^{207} \mathrm{~Pb}^{*} \\
{ }^{235} \mathrm{U}\end{array}$ & $\begin{array}{c} \pm \\
\text { (abs) }\end{array}$ & $\begin{array}{l}{ }^{206} \mathrm{~Pb}^{*} \\
{ }^{238} \mathrm{U}\end{array}$ & $\begin{array}{c} \pm \\
(\mathrm{abs})\end{array}$ & $\begin{array}{l}{ }^{207} \mathrm{~Pb}^{*} \\
{ }^{206} \mathrm{~Pb}^{*}\end{array}$ & $\begin{array}{c} \pm \\
\text { (abs) }\end{array}$ & $\begin{array}{c}{ }^{207} \mathrm{~Pb}^{*} \\
{ }^{235} \mathrm{U}\end{array}$ & $\begin{array}{c} \pm \\
\text { (abs) }\end{array}$ & $\begin{array}{c}{ }^{206} \mathrm{~Pb}^{*} \\
{ }^{238} \mathrm{U}\end{array}$ & $\begin{array}{c} \pm \\
(\mathrm{abs})\end{array}$ & $\begin{array}{l}{ }^{207} \mathrm{~Pb}^{*} \\
{ }^{206} \mathrm{~Pb}^{*}\end{array}$ & $\begin{array}{c} \pm \\
(\mathrm{abs})\end{array}$ & $\begin{array}{c}\text { Disc. } \\
(\%)\end{array}$ \\
\hline 24 & 20 & 24,634 & 3.847 & 0.226 & 0.2599 & 0.0130 & 0.1074 & 0.0028 & 1603 & 47 & 1489 & 66 & 1755 & 48 & 4.57 \\
\hline 25 & 19 & 28,777 & 4.475 & 0.279 & 0.2953 & 0.0158 & 0.1099 & 0.0029 & 1726 & 52 & 1668 & 79 & 1798 & 48 & 1.74 \\
\hline 26 & 19 & 3,516 & 3.818 & 0.240 & 0.2621 & 0.0131 & 0.1057 & 0.0032 & 1597 & 50 & 1500 & 67 & 1726 & 55 & 3.77 \\
\hline 27 & 20 & 17,050 & 3.907 & 0.228 & 0.2576 & 0.0127 & 0.1100 & 0.0029 & 1615 & 47 & 1478 & 65 & 1799 & 48 & 5.70 \\
\hline 28 & 20 & 14,630 & 3.845 & 0.225 & 0.2611 & 0.0128 & 0.1068 & 0.0028 & 1602 & 47 & 1495 & 66 & 1746 & 49 & 4.25 \\
\hline 29 & 18 & 8,117 & 3.721 & 0.226 & 0.2556 & 0.0130 & 0.1056 & 0.0029 & 1576 & 49 & 1467 & 67 & 1724 & 50 & 4.50 \\
\hline 30 & 19 & 11,898 & 3.738 & 0.221 & 0.2558 & 0.0127 & 0.1060 & 0.0028 & 1580 & 47 & 1468 & 65 & 1732 & 49 & 4.64 \\
\hline 31 & 19 & 69,251 & 3.847 & 0.225 & 0.2596 & 0.0129 & 0.1075 & 0.0028 & 1603 & 47 & 1488 & 66 & 1757 & 48 & 4.63 \\
\hline 32 & 19 & 13,252 & 4.484 & 0.273 & 0.2871 & 0.0148 & 0.1133 & 0.0030 & 1728 & 51 & 1627 & 74 & 1853 & 48 & 3.28 \\
\hline 33 & 17 & 15,776 & 3.979 & 0.240 & 0.2644 & 0.0135 & 0.1091 & 0.0029 & 1630 & 49 & 1512 & 69 & 1785 & 48 & 4.57 \\
\hline 34 & 17 & 8,236 & 4.498 & 0.278 & 0.2872 & 0.0148 & 0.1136 & 0.0031 & 1731 & 51 & 1628 & 74 & 1857 & 50 & 3.34 \\
\hline 35 & 18 & 9,899 & 4.534 & 0.288 & 0.2971 & 0.0160 & 0.1107 & 0.0030 & 1737 & 53 & 1677 & 79 & 1810 & 49 & 1.78 \\
\hline 36 & 19 & 14,802 & 3.602 & 0.207 & 0.2352 & 0.0115 & 0.1111 & 0.0029 & 1550 & 46 & 1362 & 60 & 1817 & 48 & 9.67 \\
\hline 37 & 20 & 13,210 & 3.909 & 0.253 & 0.2564 & 0.0145 & 0.1106 & 0.0029 & 1615 & 52 & 1471 & 74 & 1809 & 48 & 6.06 \\
\hline 38 & 18 & 13,930 & 4.343 & 0.293 & 0.2927 & 0.0173 & 0.1076 & 0.0029 & 1702 & 56 & 1655 & 86 & 1759 & 49 & 1.42 \\
\hline 39 & 19 & 15,324 & 3.981 & 0.238 & 0.2716 & 0.0137 & 0.1063 & 0.0028 & 1630 & 49 & 1549 & 70 & 1737 & 49 & 2.94 \\
\hline 40 & 19 & 24,033 & 4.221 & 0.267 & 0.2850 & 0.0156 & 0.1074 & 0.0028 & 1678 & 52 & 1617 & 78 & 1756 & 48 & 1.99 \\
\hline 41 & 20 & 17,788 & 4.274 & 0.257 & 0.2821 & 0.0144 & 0.1099 & 0.0029 & 1688 & 49 & 1602 & 72 & 1797 & 48 & 2.89 \\
\hline 42 & 18 & 9,325 & 3.891 & 0.240 & 0.2671 & 0.0139 & 0.1057 & 0.0029 & 1612 & 50 & 1526 & 71 & 1726 & 50 & 3.22 \\
\hline 43 & 18 & 24,415 & 4.230 & 0.255 & 0.2854 & 0.0146 & 0.1075 & 0.0028 & 1680 & 50 & 1619 & 73 & 1757 & 48 & 1.97 \\
\hline 44 & 19 & 7,431 & 4.004 & 0.243 & 0.2750 & 0.0139 & 0.1056 & 0.0029 & 1635 & 49 & 1566 & 70 & 1725 & 50 & 2.41 \\
\hline 45 & 12 & 5,879 & 3.770 & 0.237 & 0.2565 & 0.0133 & 0.1066 & 0.0031 & 1586 & 51 & 1472 & 68 & 1742 & 53 & 4.75 \\
\hline 46 & 19 & 8,211 & 4.084 & 0.248 & 0.2780 & 0.0141 & 0.1065 & 0.0029 & 1651 & 49 & 1581 & 71 & 1741 & 50 & 2.38 \\
\hline 47 & 19 & 13,346 & 4.006 & 0.238 & 0.2721 & 0.0136 & 0.1068 & 0.0028 & 1635 & 48 & 1552 & 69 & 1745 & 48 & 3.02 \\
\hline 48 & 19 & 9,429 & 4.768 & 0.317 & 0.3242 & 0.0185 & 0.1067 & 0.0028 & 1779 & 56 & 1810 & 90 & 1743 & 49 & -0.72 \\
\hline 49 & 19 & 9,707 & 4.001 & 0.242 & 0.2755 & 0.0140 & 0.1053 & 0.0028 & 1634 & 49 & 1568 & 71 & 1720 & 49 & 2.30 \\
\hline 50 & 19 & 21,830 & 4.506 & 0.275 & 0.3057 & 0.0158 & 0.1069 & 0.0028 & 1732 & 51 & 1719 & 78 & 1748 & 48 & 0.35 \\
\hline 51 & 20 & 24,551 & 4.255 & 0.255 & 0.2812 & 0.0143 & 0.1097 & 0.0029 & 1685 & 49 & 1598 & 72 & 1795 & 48 & 2.93 \\
\hline 52 & 19 & 12,891 & 4.495 & 0.286 & 0.3021 & 0.0164 & 0.1079 & 0.0029 & 1730 & 53 & 1702 & 81 & 1764 & 49 & 0.80 \\
\hline 53 & 18 & 10,055 & 3.912 & 0.235 & 0.2676 & 0.0134 & 0.1060 & 0.0029 & 1616 & 49 & 1529 & 68 & 1732 & 49 & 3.27 \\
\hline 54 & 19 & 8,204 & 3.793 & 0.239 & 0.2585 & 0.0139 & 0.1064 & 0.0028 & 1591 & 51 & 1482 & 71 & 1739 & 49 & 4.44 \\
\hline 55 & 14 & 16,936 & 3.946 & 0.241 & 0.2632 & 0.0135 & 0.1087 & 0.0030 & 1623 & 49 & 1506 & 69 & 1779 & 50 & 4.60 \\
\hline 56 & 19 & 27,476 & 3.804 & 0.222 & 0.2544 & 0.0126 & 0.1084 & 0.0029 & 1594 & 47 & 1461 & 65 & 1773 & 48 & 5.63 \\
\hline 57 & 6 & 1,238 & 3.489 & 0.382 & 0.2633 & 0.0223 & 0.0961 & 0.0045 & 1525 & 86 & 1507 & 114 & 1550 & 87 & 0.68 \\
\hline 58 & 20 & 13,377 & 3.743 & 0.219 & 0.2546 & 0.0126 & 0.1066 & 0.0028 & 1581 & 47 & 1462 & 65 & 1743 & 48 & 5.00 \\
\hline 59 & 19 & 12,322 & 3.946 & 0.235 & 0.2669 & 0.0134 & 0.1072 & 0.0029 & 1623 & 48 & 1525 & 68 & 1753 & 49 & 3.71 \\
\hline 60 & 20 & 14,629 & 3.755 & 0.221 & 0.2593 & 0.0128 & 0.1050 & 0.0028 & 1583 & 47 & 1486 & 65 & 1715 & 50 & 3.89 \\
\hline 61 & 18 & 19,418 & 3.801 & 0.223 & 0.2535 & 0.0125 & 0.1087 & 0.0029 & 1593 & 47 & 1457 & 64 & 1779 & 48 & 5.85 \\
\hline 62 & 19 & 10,304 & 3.919 & 0.235 & 0.2677 & 0.0134 & 0.1062 & 0.0029 & 1618 & 48 & 1529 & 68 & 1735 & 50 & 3.32 \\
\hline 63 & 19 & 22,453 & 3.793 & 0.222 & 0.2565 & 0.0127 & 0.1073 & 0.0028 & 1591 & 47 & 1472 & 65 & 1753 & 48 & 4.95 \\
\hline 64 & 19 & 16,855 & 4.016 & 0.238 & 0.2670 & 0.0134 & 0.1091 & 0.0029 & 1637 & 48 & 1525 & 68 & 1784 & 48 & 4.26 \\
\hline 65 & 20 & 21,967 & 4.126 & 0.243 & 0.2689 & 0.0134 & 0.1113 & 0.0029 & 1659 & 48 & 1535 & 68 & 1820 & 48 & 4.67 \\
\hline 66 & 15 & 6,958 & 3.452 & 0.246 & 0.2384 & 0.0149 & 0.1050 & 0.0029 & 1516 & 56 & 1378 & 78 & 1715 & 51 & 6.73 \\
\hline 67 & 20 & 20,885 & 3.957 & 0.238 & 0.2615 & 0.0135 & 0.1097 & 0.0029 & 1625 & 49 & 1498 & 69 & 1795 & 48 & 5.12 \\
\hline 68 & 17 & 11,739 & 3.474 & 0.250 & 0.2330 & 0.0150 & 0.1081 & 0.0029 & 1521 & 57 & 1350 & 78 & 1768 & 49 & 8.88 \\
\hline 69 & 14 & 5,994 & 3.682 & 0.246 & 0.2557 & 0.0145 & 0.1045 & 0.0030 & 1568 & 53 & 1468 & 74 & 1705 & 52 & 4.14 \\
\hline
\end{tabular}


Isotopic ratios (corrected)

Ages (Ma)

\begin{tabular}{|c|c|c|c|c|c|c|c|c|c|c|c|c|c|c|c|}
\hline \# & $\mathrm{N}$ & $\frac{{ }^{206} \mathrm{~Pb}}{{ }^{204} \mathrm{~Pb}^{\dagger}}$ & $\begin{array}{c}{ }^{207} \mathrm{~Pb}^{*} \\
{ }^{235} \mathrm{U}\end{array}$ & $\begin{array}{c} \pm \\
(\mathrm{abs})\end{array}$ & $\begin{array}{c}{ }^{206} \mathrm{~Pb}^{*} \\
{ }^{238} \mathrm{U} \\
\end{array}$ & $\begin{array}{c} \pm \\
\text { (abs) }\end{array}$ & $\begin{array}{c}{ }^{207} \mathrm{~Pb}^{*} \\
{ }^{206} \mathrm{~Pb}^{*}\end{array}$ & $\begin{array}{c} \pm \\
(\mathrm{abs})\end{array}$ & $\begin{array}{l}{ }^{207} \mathrm{~Pb}^{*} \\
{ }^{235} \mathrm{U} \\
\end{array}$ & $\begin{array}{c} \pm \\
\text { (abs) }\end{array}$ & $\begin{array}{l}{ }^{206} \mathrm{~Pb}^{*} \\
{ }^{238} \mathrm{U} \\
\end{array}$ & $\begin{array}{c} \pm \\
\text { (abs) }\end{array}$ & $\begin{array}{l}{ }^{207} \mathrm{~Pb}^{*} \\
{ }^{206} \mathrm{~Pb}^{*}\end{array}$ & $\begin{array}{c} \pm \\
(\mathrm{abs})\end{array}$ & $\begin{array}{c}\text { Disc. } \\
(\%)\end{array}$ \\
\hline 70 & 20 & 14,790 & 4.206 & 0.258 & 0.2840 & 0.0148 & 0.1074 & 0.0028 & 1675 & 50 & 1612 & 74 & 1756 & 48 & 2.08 \\
\hline 71 & 16 & 8,907 & 4.203 & 0.261 & 0.2781 & 0.0145 & 0.1096 & 0.0030 & 1675 & 51 & 1582 & 73 & 1793 & 49 & 3.20 \\
\hline 72 & 18 & 21,048 & 5.916 & 0.461 & 0.3901 & 0.0271 & 0.1100 & 0.0029 & 1964 & 68 & 2123 & 126 & 1799 & 48 & -2.33 \\
\hline 73 & 16 & 11,013 & 4.062 & 0.256 & 0.2760 & 0.0148 & 0.1067 & 0.0029 & 1647 & 51 & 1571 & 75 & 1744 & 50 & 2.63 \\
\hline 74 & 20 & 17,358 & 3.993 & 0.237 & 0.2681 & 0.0134 & 0.1080 & 0.0028 & 1633 & 48 & 1531 & 68 & 1766 & 48 & 3.80 \\
\hline 75 & 17 & 19,675 & 4.432 & 0.309 & 0.2993 & 0.0184 & 0.1074 & 0.0029 & 1718 & 58 & 1688 & 91 & 1755 & 49 & 0.87 \\
\hline 76 & 17 & 11,644 & 5.822 & 0.453 & 0.3845 & 0.0265 & 0.1098 & 0.0029 & 1950 & 67 & 2097 & 123 & 1797 & 48 & -2.24 \\
\hline 77 & 18 & 11,390 & 4.069 & 0.256 & 0.2744 & 0.0145 & 0.1075 & 0.0029 & 1648 & 51 & 1563 & 73 & 1758 & 49 & 3.01 \\
\hline 78 & 19 & 13,110 & 11.844 & 0.927 & 0.4718 & 0.0316 & 0.1821 & 0.0048 & 2592 & 73 & 2492 & 138 & 2672 & 43 & 1.07 \\
\hline 79 & 19 & 21,184 & 5.522 & 0.410 & 0.3655 & 0.0238 & 0.1096 & 0.0029 & 1904 & 64 & 2008 & 112 & 1793 & 48 & -1.81 \\
\hline 80 & 14 & 4,526 & 13.510 & 1.441 & 0.5236 & 0.0493 & 0.1871 & 0.0051 & 2716 & 101 & 2715 & 209 & 2717 & 45 & 0.01 \\
\hline 81 & 19 & 10,907 & 3.149 & 0.480 & 0.2139 & 0.0315 & 0.1068 & 0.0029 & 1445 & 117 & 1250 & 168 & 1745 & 49 & 12.21 \\
\hline 82 & 18 & 9,337 & 3.844 & 0.238 & 0.2540 & 0.0133 & 0.1098 & 0.0029 & 1602 & 50 & 1459 & 68 & 1795 & 49 & 6.13 \\
\hline 83 & 19 & 12,806 & 4.297 & 0.266 & 0.2754 & 0.0144 & 0.1132 & 0.0030 & 1693 & 51 & 1568 & 73 & 1851 & 48 & 4.48 \\
\hline 84 & 17 & 45,395 & 4.016 & 0.248 & 0.2628 & 0.0138 & 0.1108 & 0.0029 & 1637 & 50 & 1504 & 71 & 1813 & 48 & 5.28 \\
\hline 85 & 18 & 10,516 & 3.795 & 0.233 & 0.2591 & 0.0133 & 0.1062 & 0.0029 & 1592 & 49 & 1485 & 68 & 1736 & 49 & 4.30 \\
\hline 86 & 18 & 18,811 & 3.734 & 0.227 & 0.2539 & 0.0130 & 0.1067 & 0.0028 & 1579 & 49 & 1459 & 67 & 1743 & 49 & 5.10 \\
\hline 87 & 19 & 13,004 & 4.032 & 0.253 & 0.2751 & 0.0145 & 0.1063 & 0.0029 & 1641 & 51 & 1567 & 73 & 1737 & 49 & 2.59 \\
\hline 88 & 18 & 9,053 & 7.799 & 0.596 & 0.3535 & 0.0238 & 0.1600 & 0.0042 & 2208 & 69 & 1951 & 113 & 2456 & 45 & 5.75 \\
\hline 89 & 19 & 23,749 & 4.615 & 0.300 & 0.2986 & 0.0166 & 0.1121 & 0.0029 & 1752 & 54 & 1684 & 83 & 1834 & 47 & 1.99 \\
\hline 90 & 20 & 16,680 & 4.263 & 0.277 & 0.2925 & 0.0163 & 0.1057 & 0.0028 & 1686 & 53 & 1654 & 81 & 1727 & 48 & 0.97 \\
\hline 91 & 19 & 16,871 & 4.413 & 0.312 & 0.3000 & 0.0186 & 0.1067 & 0.0028 & 1715 & 58 & 1691 & 92 & 1743 & 48 & 0.66 \\
\hline 92 & 17 & 9,890 & 4.692 & 0.325 & 0.3193 & 0.0189 & 0.1066 & 0.0029 & 1766 & 58 & 1786 & 93 & 1742 & 49 & -0.50 \\
\hline 93 & 15 & 11,909 & 4.440 & 0.329 & 0.3062 & 0.0199 & 0.1052 & 0.0029 & 1720 & 61 & 1722 & 98 & 1717 & 50 & -0.05 \\
\hline 94 & 17 & 15,228 & 4.536 & 0.299 & 0.3072 & 0.0173 & 0.1071 & 0.0028 & 1738 & 55 & 1727 & 85 & 1751 & 49 & 0.29 \\
\hline 95 & 16 & 10,909 & 5.117 & 0.374 & 0.3480 & 0.0220 & 0.1066 & 0.0029 & 1839 & 62 & 1925 & 105 & 1743 & 49 & -1.68 \\
\hline 96 & 19 & 13,130 & 5.595 & 0.395 & 0.3844 & 0.0231 & 0.1056 & 0.0028 & 1915 & 61 & 2097 & 108 & 1724 & 49 & -2.71 \\
\hline 97 & 17 & 15,808 & 5.645 & 0.447 & 0.3724 & 0.0261 & 0.1100 & 0.0029 & 1923 & 68 & 2041 & 123 & 1799 & 49 & -1.94 \\
\hline 98 & 18 & 15,858 & 4.940 & 0.347 & 0.3364 & 0.0205 & 0.1065 & 0.0028 & 1809 & 59 & 1870 & 99 & 1740 & 48 & -1.29 \\
\hline 99 & 20 & 21,817 & 4.181 & 0.258 & 0.2777 & 0.0145 & 0.1092 & 0.0029 & 1670 & 50 & 1580 & 73 & 1786 & 48 & 3.14 \\
\hline 100 & 19 & 21,380 & 5.359 & 0.385 & 0.3627 & 0.0226 & 0.1072 & 0.0028 & 1878 & 61 & 1995 & 107 & 1752 & 48 & -2.04 \\
\hline 101 & 17 & 11,964 & 4.007 & 0.253 & 0.2740 & 0.0146 & 0.1061 & 0.0028 & 1636 & 51 & 1561 & 74 & 1733 & 49 & 2.63 \\
\hline 102 & 18 & 17,370 & 5.129 & 0.347 & 0.3475 & 0.0200 & 0.1071 & 0.0028 & 1841 & 57 & 1923 & 96 & 1750 & 49 & -1.60 \\
\hline
\end{tabular}

\begin{tabular}{|c|c|c|c|c|c|c|c|c|c|c|c|c|c|c|c|}
\hline 1 & 20 & 8,885 & 4.602 & 0.321 & 0.3161 & 0.0185 & 0.1056 & 0.0028 & 1750 & 58 & 1771 & 91 & 1725 & 49 & -0.52 \\
\hline 2 & 19 & 8,885 & 4.826 & 0.349 & 0.3277 & 0.0200 & 0.1068 & 0.0028 & 1789 & 61 & 1827 & 97 & 1746 & 49 & -0.86 \\
\hline 3 & 17 & 5,975 & 5.078 & 0.678 & 0.3355 & 0.0421 & 0.1098 & 0.0030 & 1832 & 113 & 1865 & 203 & 1796 & 49 & -0.70 \\
\hline 4 & 18 & 14,781 & 5.874 & 0.589 & 0.4002 & 0.0364 & 0.1064 & 0.0028 & 1957 & 87 & 2170 & 168 & 1739 & 49 & -2.85 \\
\hline 5 & 19 & 14,301 & 4.131 & 0.279 & 0.2822 & 0.0160 & 0.1062 & 0.0028 & 1660 & 55 & 1603 & 81 & 1735 & 49 & 1.91 \\
\hline 6 & 19 & 11,076 & 5.037 & 0.362 & 0.3317 & 0.0202 & 0.1102 & 0.0029 & 1826 & 61 & 1846 & 98 & 1802 & 48 & -0.47 \\
\hline 7 & 19 & 21,492 & 5.175 & 0.371 & 0.3489 & 0.0212 & 0.1076 & 0.0028 & 1849 & 61 & 1929 & 101 & 1759 & 48 & -1.57 \\
\hline 8 & 17 & 6,958 & 4.982 & 0.394 & 0.3295 & 0.0225 & 0.1097 & 0.0030 & 1816 & 67 & 1836 & 109 & 1794 & 49 & -0.45 \\
\hline 9 & 20 & 13,815 & 4.751 & 0.338 & 0.3151 & 0.0190 & 0.1094 & 0.0029 & 1776 & 60 & 1766 & 93 & 1789 & 49 & 0.27 \\
\hline 10 & 20 & 6,618 & 5.796 & 0.449 & 0.3897 & 0.0251 & 0.1079 & 0.0030 & 1946 & 67 & 2121 & 116 & 1764 & 50 & -2.55 \\
\hline 11 & 19 & 14,065 & 5.472 & 0.457 & 0.3624 & 0.0267 & 0.1095 & 0.0029 & 1896 & 72 & 1994 & 126 & 1791 & 48 & -1.73 \\
\hline
\end{tabular}


Isotopic ratios (corrected)

Ages (Ma)

\begin{tabular}{|c|c|c|c|c|c|c|c|c|c|c|c|c|c|c|c|}
\hline$\#$ & $\mathrm{~N}$ & $\frac{{ }^{206} \mathrm{~Pb}}{{ }^{204} \mathrm{~Pb}^{\dagger}}$ & $\begin{array}{l}{ }^{207} \mathrm{~Pb}^{*} \\
{ }^{235} \mathrm{U}\end{array}$ & $\begin{array}{c} \pm \\
\text { (abs) }\end{array}$ & $\begin{array}{l}{ }^{206} \mathrm{~Pb}^{*} \\
{ }^{238} \mathrm{U}\end{array}$ & $\begin{array}{c} \pm \\
\text { (abs) }\end{array}$ & $\begin{array}{l}{ }^{207} \mathrm{~Pb}^{*} \\
{ }^{206} \mathrm{~Pb}^{*}\end{array}$ & $\begin{array}{c} \pm \\
\text { (abs) }\end{array}$ & $\begin{array}{c}{ }^{207} \mathrm{~Pb}^{*} \\
{ }^{235} \mathrm{U}\end{array}$ & $\begin{array}{c} \pm \\
\text { (abs) }\end{array}$ & $\begin{array}{c}{ }^{206} \mathrm{~Pb}^{*} \\
{ }^{238} \mathrm{U}\end{array}$ & $\begin{array}{c} \pm \\
(\mathrm{abs})\end{array}$ & $\begin{array}{l}{ }^{207} \mathrm{~Pb}^{*} \\
{ }^{206} \mathrm{~Pb}^{*}\end{array}$ & $\begin{array}{c} \pm \\
(\mathrm{abs})\end{array}$ & $\begin{array}{c}\text { Disc. } \\
\text { (\%) }\end{array}$ \\
\hline 12 & 20 & 11,750 & 4.950 & 0.363 & 0.3396 & 0.0213 & 0.1057 & 0.0028 & 1811 & 62 & 1885 & 102 & 1727 & 48 & -1.53 \\
\hline 13 & 19 & 16,239 & 4.295 & 0.293 & 0.2956 & 0.0171 & 0.1054 & 0.0028 & 1692 & 56 & 1670 & 85 & 1721 & 48 & 0.67 \\
\hline 14 & 17 & 6,821 & 4.748 & 0.373 & 0.3265 & 0.0221 & 0.1055 & 0.0029 & 1776 & 66 & 1821 & 107 & 1723 & 50 & -1.05 \\
\hline 15 & 19 & 13,113 & 4.759 & 0.348 & 0.3252 & 0.0203 & 0.1061 & 0.0028 & 1778 & 61 & 1815 & 99 & 1734 & 49 & -0.87 \\
\hline 16 & 11 & 4,430 & 5.713 & 0.471 & 0.3576 & 0.0247 & 0.1159 & 0.0034 & 1933 & 71 & 1971 & 117 & 1894 & 53 & -0.70 \\
\hline 17 & 19 & 16,641 & 5.164 & 0.387 & 0.3545 & 0.0227 & 0.1056 & 0.0028 & 1847 & 64 & 1956 & 108 & 1726 & 48 & -2.03 \\
\hline 18 & 16 & 6,613 & 3.218 & 0.242 & 0.2185 & 0.0144 & 0.1068 & 0.0029 & 1461 & 58 & 1274 & 76 & 1746 & 50 & 11.22 \\
\hline 19 & 19 & 2,394 & 3.142 & 0.280 & 0.2131 & 0.0166 & 0.1069 & 0.0030 & 1443 & 69 & 1245 & 88 & 1748 & 51 & 12.49 \\
\hline 20 & 19 & 6,434 & 3.973 & 0.331 & 0.2647 & 0.0196 & 0.1089 & 0.0029 & 1629 & 68 & 1514 & 100 & 1781 & 48 & 4.46 \\
\hline 21 & 20 & 15,981 & 5.566 & 0.402 & 0.3533 & 0.0216 & 0.1143 & 0.0030 & 1911 & 62 & 1950 & 103 & 1868 & 48 & -0.76 \\
\hline 22 & 19 & 12,174 & 5.069 & 0.373 & 0.3450 & 0.0215 & 0.1066 & 0.0028 & 1831 & 62 & 1911 & 103 & 1742 & 49 & -1.59 \\
\hline 23 & 19 & 32,579 & 5.553 & 0.466 & 0.3794 & 0.0281 & 0.1062 & 0.0028 & 1909 & 72 & 2073 & 131 & 1734 & 48 & -2.55 \\
\hline 24 & 18 & 3,601 & 5.461 & 0.509 & 0.3739 & 0.0304 & 0.1059 & 0.0029 & 1894 & 80 & 2048 & 143 & 1730 & 50 & -2.47 \\
\hline 25 & 16 & 6,663 & 4.229 & 0.309 & 0.2933 & 0.0182 & 0.1046 & 0.0029 & 1680 & 60 & 1658 & 91 & 1707 & 50 & 0.64 \\
\hline 26 & 20 & 17,229 & 4.662 & 0.328 & 0.3201 & 0.0191 & 0.1056 & 0.0028 & 1760 & 59 & 1790 & 93 & 1725 & 48 & -0.72 \\
\hline 27 & 19 & 3,690 & 3.810 & 0.274 & 0.2585 & 0.0157 & 0.1069 & 0.0029 & 1595 & 58 & 1482 & 80 & 1747 & 50 & 4.58 \\
\hline 28 & 19 & 12,135 & 4.616 & 0.327 & 0.3188 & 0.0191 & 0.1050 & 0.0028 & 1752 & 59 & 1784 & 94 & 1714 & 49 & -0.77 \\
\hline 29 & 17 & 13,921 & 4.872 & 0.353 & 0.3344 & 0.0205 & 0.1057 & 0.0028 & 1797 & 61 & 1860 & 99 & 1726 & 49 & -1.34 \\
\hline 30 & 19 & 20,921 & 4.744 & 0.336 & 0.3132 & 0.0189 & 0.1098 & 0.0029 & 1775 & 59 & 1757 & 93 & 1797 & 48 & 0.48 \\
\hline 31 & 19 & 39,671 & 4.732 & 0.319 & 0.2975 & 0.0170 & 0.1154 & 0.0030 & 1773 & 57 & 1679 & 85 & 1886 & 47 & 2.83 \\
\hline 32 & 20 & 41,369 & 4.443 & 0.298 & 0.2925 & 0.0166 & 0.1102 & 0.0029 & 1720 & 56 & 1654 & 83 & 1802 & 48 & 2.04 \\
\hline 33 & 20 & 38,612 & 4.140 & 0.277 & 0.2816 & 0.0160 & 0.1066 & 0.0028 & 1662 & 55 & 1599 & 81 & 1742 & 48 & 2.08 \\
\hline 34 & 19 & 7,349 & 5.044 & 0.387 & 0.3463 & 0.0226 & 0.1056 & 0.0028 & 1827 & 65 & 1917 & 108 & 1726 & 49 & -1.77 \\
\hline 35 & 19 & 19,482 & 4.814 & 0.340 & 0.3217 & 0.0194 & 0.1085 & 0.0029 & 1787 & 59 & 1798 & 94 & 1775 & 48 & -0.26 \\
\hline 36 & 18 & 19,761 & 4.988 & 0.368 & 0.3418 & 0.0216 & 0.1058 & 0.0028 & 1817 & 62 & 1895 & 104 & 1729 & 48 & -1.59 \\
\hline 37 & 15 & 15,800 & 5.937 & 0.575 & 0.3903 & 0.0341 & 0.1103 & 0.0030 & 1967 & 84 & 2124 & 158 & 1805 & 49 & -2.30 \\
\hline 38 & 20 & 3,353 & 2.972 & 0.213 & 0.1843 & 0.0113 & 0.1169 & 0.0032 & 1401 & 54 & 1091 & 61 & 1910 & 49 & 27.79 \\
\hline 39 & 16 & 12,015 & 4.510 & 0.319 & 0.3115 & 0.0186 & 0.1050 & 0.0028 & 1733 & 59 & 1748 & 91 & 1714 & 49 & -0.40 \\
\hline 40 & 18 & 21,406 & 4.701 & 0.330 & 0.3206 & 0.0191 & 0.1064 & 0.0028 & 1767 & 59 & 1793 & 93 & 1738 & 48 & -0.61 \\
\hline 41 & 15 & 9,684 & 5.598 & 0.502 & 0.3830 & 0.0303 & 0.1060 & 0.0029 & 1916 & 77 & 2090 & 141 & 1732 & 50 & -2.64 \\
\hline 42 & 20 & 11,180 & 5.357 & 0.398 & 0.3656 & 0.0229 & 0.1063 & 0.0028 & 1878 & 64 & 2009 & 108 & 1736 & 49 & -2.24 \\
\hline 43 & 19 & 6,522 & 5.289 & 0.404 & 0.3598 & 0.0233 & 0.1066 & 0.0028 & 1867 & 65 & 1981 & 110 & 1742 & 49 & -2.04 \\
\hline 44 & 14 & 5,191 & 4.453 & 0.400 & 0.3056 & 0.0243 & 0.1057 & 0.0029 & 1722 & 75 & 1719 & 120 & 1726 & 51 & 0.09 \\
\hline 45 & 14 & 5,103 & 5.634 & 0.473 & 0.3847 & 0.0275 & 0.1062 & 0.0029 & 1921 & 72 & 2098 & 128 & 1735 & 51 & -2.64 \\
\hline 46 & 18 & 23,716 & 4.859 & 0.356 & 0.3335 & 0.0210 & 0.1057 & 0.0028 & 1795 & 62 & 1855 & 101 & 1726 & 49 & -1.30 \\
\hline 47 & 13 & 13,542 & 5.756 & 0.464 & 0.3800 & 0.0262 & 0.1099 & 0.0030 & 1940 & 70 & 2076 & 122 & 1797 & 50 & -2.14 \\
\hline 48 & 17 & 19,046 & 5.964 & 0.486 & 0.4085 & 0.0286 & 0.1059 & 0.0028 & 1971 & 71 & 2208 & 131 & 1730 & 49 & -3.00 \\
\hline 49 & 20 & 18,916 & 5.899 & 0.484 & 0.4008 & 0.0285 & 0.1067 & 0.0028 & 1961 & 71 & 2173 & 131 & 1745 & 48 & -2.83 \\
\hline 50 & 19 & 24,098 & 5.156 & 0.388 & 0.3374 & 0.0220 & 0.1108 & 0.0029 & 1845 & 64 & 1874 & 106 & 1813 & 48 & -0.62 \\
\hline 51 & 16 & 10,318 & 4.381 & 0.315 & 0.2981 & 0.0182 & 0.1066 & 0.0029 & 1709 & 59 & 1682 & 90 & 1742 & 49 & 0.78 \\
\hline 52 & 14 & 6,932 & 4.634 & 0.367 & 0.3208 & 0.0216 & 0.1048 & 0.0030 & 1755 & 66 & 1793 & 105 & 1710 & 52 & -0.91 \\
\hline 53 & 17 & 17,490 & 4.382 & 0.326 & 0.3002 & 0.0194 & 0.1059 & 0.0028 & 1709 & 61 & 1692 & 96 & 1729 & 49 & 0.47 \\
\hline 54 & 13 & 2,437 & 3.763 & 0.331 & 0.2574 & 0.0194 & 0.1060 & 0.0031 & 1585 & 71 & 1477 & 100 & 1732 & 53 & 4.44 \\
\hline 55 & 20 & 18,933 & 5.325 & 0.396 & 0.3336 & 0.0213 & 0.1158 & 0.0031 & 1873 & 63 & 1856 & 103 & 1892 & 48 & 0.38 \\
\hline 56 & 19 & 29,524 & 4.688 & 0.323 & 0.2933 & 0.0172 & 0.1159 & 0.0030 & 1765 & 58 & 1658 & 86 & 1894 & 47 & 3.33 \\
\hline 57 & 19 & 13,679 & 4.327 & 0.305 & 0.2952 & 0.0178 & 0.1063 & 0.0028 & 1698 & 58 & 1667 & 88 & 1737 & 49 & 0.92 \\
\hline
\end{tabular}


Isotopic ratios (corrected)

Ages (Ma)

\begin{tabular}{|c|c|c|c|c|c|c|c|c|c|c|c|c|c|c|c|}
\hline \# & $\mathrm{N}$ & $\frac{{ }^{206} \mathrm{~Pb}}{{ }^{204} \mathrm{~Pb}^{\dagger}}$ & $\begin{array}{c}{ }^{207} \mathrm{~Pb}^{*} \\
{ }^{235} \mathrm{U}\end{array}$ & $\begin{array}{c} \pm \\
\text { (abs) }\end{array}$ & $\begin{array}{l}{ }^{206} \mathrm{~Pb}^{*} \\
{ }^{238} \mathrm{U}\end{array}$ & $\begin{array}{c} \pm \\
\text { (abs) }\end{array}$ & $\begin{array}{l}{ }^{207} \mathrm{~Pb}^{*} \\
{ }^{206} \mathrm{~Pb}^{*}\end{array}$ & $\begin{array}{c} \pm \\
\text { (abs) }\end{array}$ & $\begin{array}{c}{ }^{207} \mathrm{~Pb}^{*} \\
{ }^{235} \mathrm{U}\end{array}$ & $\begin{array}{c} \pm \\
\text { (abs) }\end{array}$ & $\begin{array}{c}{ }^{206} \mathrm{~Pb}^{*} \\
{ }^{238} \mathrm{U}\end{array}$ & $\begin{array}{c} \pm \\
(\mathrm{abs})\end{array}$ & $\begin{array}{l}{ }^{207} \mathrm{~Pb}^{*} \\
{ }^{206} \mathrm{~Pb}^{*}\end{array}$ & $\begin{array}{c} \pm \\
(\mathrm{abs})\end{array}$ & $\begin{array}{c}\text { Disc. } \\
\text { (\%) }\end{array}$ \\
\hline 58 & 19 & 9,703 & 4.223 & 0.293 & 0.2603 & 0.0154 & 0.1177 & 0.0031 & 1679 & 57 & 1492 & 79 & 1921 & 47 & 7.79 \\
\hline 59 & 12 & 9,089 & 4.556 & 0.339 & 0.2934 & 0.0185 & 0.1126 & 0.0032 & 1741 & 62 & 1659 & 92 & 1842 & 51 & 2.54 \\
\hline 60 & 13 & 6,896 & 4.522 & 0.347 & 0.3199 & 0.0206 & 0.1025 & 0.0030 & 1735 & 64 & 1789 & 100 & 1671 & 54 & -1.29 \\
\hline 61 & 18 & 5,178 & 6.010 & 0.555 & 0.4105 & 0.0330 & 0.1062 & 0.0029 & 1977 & 80 & 2217 & 151 & 1735 & 49 & -3.00 \\
\hline 62 & 20 & 13,007 & 4.336 & 0.300 & 0.2944 & 0.0172 & 0.1068 & 0.0028 & 1700 & 57 & 1663 & 86 & 1746 & 49 & 1.10 \\
\hline 63 & 20 & 14,936 & 5.064 & 0.371 & 0.3450 & 0.0215 & 0.1065 & 0.0028 & 1830 & 62 & 1911 & 103 & 1740 & 48 & -1.61 \\
\hline 64 & 20 & 12,214 & 4.894 & 0.349 & 0.3333 & 0.0200 & 0.1065 & 0.0029 & 1801 & 60 & 1854 & 97 & 1740 & 49 & -1.16 \\
\hline 65 & 19 & 56,511 & 4.989 & 0.341 & 0.3057 & 0.0178 & 0.1184 & 0.0031 & 1817 & 58 & 1719 & 88 & 1932 & 47 & 2.78 \\
\hline 66 & 19 & 18,926 & 4.433 & 0.301 & 0.2939 & 0.0169 & 0.1094 & 0.0029 & 1718 & 56 & 1661 & 84 & 1789 & 48 & 1.74 \\
\hline 67 & 20 & 32,755 & 4.834 & 0.339 & 0.3202 & 0.0192 & 0.1095 & 0.0029 & 1791 & 59 & 1791 & 94 & 1791 & 48 & 0.00 \\
\hline 68 & 19 & 15,225 & 6.079 & 0.514 & 0.3839 & 0.0285 & 0.1149 & 0.0030 & 1987 & 74 & 2094 & 133 & 1878 & 48 & -1.66 \\
\hline 69 & 20 & 16,102 & 13.316 & 1.203 & 0.4771 & 0.0369 & 0.2024 & 0.0053 & 2702 & 85 & 2515 & 161 & 2846 & 43 & 2.03 \\
\hline 70 & 17 & 21,386 & 4.783 & 0.346 & 0.3295 & 0.0203 & 0.1053 & 0.0028 & 1782 & 61 & 1836 & 99 & 1720 & 49 & -1.20 \\
\hline 71 & 20 & 15,920 & 4.242 & 0.285 & 0.2812 & 0.0160 & 0.1094 & 0.0029 & 1682 & 55 & 1597 & 80 & 1790 & 48 & 2.86 \\
\hline 72 & 20 & 15,660 & 4.802 & 0.355 & 0.3312 & 0.0211 & 0.1051 & 0.0028 & 1785 & 62 & 1844 & 102 & 1717 & 48 & -1.31 \\
\hline 73 & 18 & 16,875 & 4.419 & 0.303 & 0.2852 & 0.0166 & 0.1124 & 0.0030 & 1716 & 57 & 1617 & 83 & 1838 & 48 & 3.24 \\
\hline 74 & 20 & 13,866 & 4.764 & 0.349 & 0.3187 & 0.0201 & 0.1084 & 0.0028 & 1779 & 61 & 1784 & 98 & 1773 & 48 & -0.12 \\
\hline 75 & 18 & 30,996 & 11.413 & 0.942 & 0.4431 & 0.0312 & 0.1868 & 0.0049 & 2557 & 77 & 2364 & 140 & 2714 & 43 & 2.50 \\
\hline 76 & 17 & 9,285 & 5.021 & 0.389 & 0.3334 & 0.0223 & 0.1092 & 0.0029 & 1823 & 66 & 1855 & 108 & 1787 & 49 & -0.70 \\
\hline 77 & 19 & 3,565 & 8.886 & 0.967 & 0.3696 & 0.0368 & 0.1744 & 0.0047 & 2326 & 99 & 2027 & 173 & 2600 & 45 & 6.20 \\
\hline 78 & 20 & 6,033 & 4.434 & 0.340 & 0.3083 & 0.0199 & 0.1043 & 0.0030 & 1719 & 64 & 1732 & 98 & 1702 & 53 & -0.35 \\
\hline 79 & 19 & 24,643 & 4.881 & 0.364 & 0.3355 & 0.0216 & 0.1055 & 0.0028 & 1799 & 63 & 1865 & 104 & 1723 & 48 & -1.41 \\
\hline 80 & 19 & 14,469 & 4.771 & 0.348 & 0.3295 & 0.0206 & 0.1050 & 0.0028 & 1780 & 61 & 1836 & 100 & 1715 & 48 & -1.25 \\
\hline 81 & 20 & 58,012 & 4.069 & 0.269 & 0.2686 & 0.0151 & 0.1099 & 0.0029 & 1648 & 54 & 1534 & 77 & 1797 & 48 & 4.30 \\
\hline 82 & 10 & 11,277 & 4.140 & 0.297 & 0.2673 & 0.0161 & 0.1123 & 0.0032 & 1662 & 59 & 1527 & 82 & 1838 & 52 & 5.19 \\
\hline 83 & 18 & 9,522 & 4.506 & 0.322 & 0.2806 & 0.0172 & 0.1165 & 0.0031 & 1732 & 59 & 1594 & 86 & 1903 & 48 & 4.78 \\
\hline 84 & 19 & 36,108 & 4.658 & 0.324 & 0.3102 & 0.0184 & 0.1089 & 0.0029 & 1760 & 58 & 1741 & 90 & 1782 & 48 & 0.48 \\
\hline 85 & 19 & 27,229 & 3.784 & 0.249 & 0.2605 & 0.0145 & 0.1054 & 0.0028 & 1589 & 53 & 1492 & 74 & 1721 & 48 & 3.86 \\
\hline 86 & 18 & 12,089 & 4.848 & 0.343 & 0.3032 & 0.0182 & 0.1160 & 0.0031 & 1793 & 60 & 1707 & 90 & 1895 & 48 & 2.48 \\
\hline 87 & 16 & 8,862 & 4.688 & 0.356 & 0.3216 & 0.0207 & 0.1057 & 0.0030 & 1765 & 64 & 1797 & 101 & 1727 & 51 & -0.77 \\
\hline 88 & 20 & 11,716 & 4.924 & 0.353 & 0.3391 & 0.0205 & 0.1053 & 0.0028 & 1806 & 61 & 1882 & 99 & 1720 & 49 & -1.58 \\
\hline 89 & 10 & 10,465 & 4.256 & 0.377 & 0.2950 & 0.0232 & 0.1046 & 0.0030 & 1685 & 73 & 1667 & 115 & 1708 & 53 & 0.54 \\
\hline 90 & 17 & 13,454 & 4.665 & 0.328 & 0.3154 & 0.0187 & 0.1073 & 0.0029 & 1761 & 59 & 1767 & 92 & 1754 & 49 & -0.16 \\
\hline 91 & 19 & 34,239 & 5.691 & 0.449 & 0.3791 & 0.0259 & 0.1089 & 0.0029 & 1930 & 68 & 2072 & 121 & 1781 & 48 & -2.23 \\
\hline 92 & 20 & 19,622 & 4.067 & 0.272 & 0.2755 & 0.0156 & 0.1071 & 0.0028 & 1648 & 54 & 1569 & 79 & 1750 & 48 & 2.77 \\
\hline 93 & 20 & 15,424 & 4.132 & 0.276 & 0.2789 & 0.0157 & 0.1074 & 0.0028 & 1661 & 55 & 1586 & 79 & 1756 & 49 & 2.54 \\
\hline 94 & 19 & 4,242 & 5.100 & 0.423 & 0.3418 & 0.0232 & 0.1082 & 0.0034 & 1836 & 70 & 1895 & 111 & 1770 & 58 & -1.22 \\
\hline 95 & 19 & 20,791 & 4.537 & 0.314 & 0.2999 & 0.0176 & 0.1097 & 0.0029 & 1738 & 58 & 1691 & 87 & 1794 & 48 & 1.34 \\
\hline 96 & 19 & 21,500 & 3.789 & 0.250 & 0.2592 & 0.0144 & 0.1060 & 0.0028 & 1590 & 53 & 1486 & 74 & 1732 & 48 & 4.21 \\
\hline 97 & 20 & 20,917 & 4.023 & 0.267 & 0.2715 & 0.0153 & 0.1075 & 0.0028 & 1639 & 54 & 1548 & 77 & 1757 & 48 & 3.29 \\
\hline 98 & 20 & 16,694 & 4.966 & 0.362 & 0.3396 & 0.0212 & 0.1061 & 0.0028 & 1814 & 62 & 1885 & 102 & 1733 & 48 & -1.48 \\
\hline 99 & 19 & 8,954 & 4.824 & 0.346 & 0.3214 & 0.0195 & 0.1088 & 0.0029 & 1789 & 60 & 1797 & 95 & 1780 & 49 & -0.18 \\
\hline 100 & 11 & 4,645 & 5.032 & 0.571 & 0.3434 & 0.0354 & 0.1063 & 0.0031 & 1825 & 96 & 1903 & 170 & 1736 & 53 & -1.58 \\
\hline 101 & 18 & 11,983 & 5.201 & 0.403 & 0.3437 & 0.0231 & 0.1098 & 0.0029 & 1853 & 66 & 1904 & 111 & 1795 & 48 & -1.05 \\
\hline 102 & 19 & 16,077 & 4.654 & 0.333 & 0.3201 & 0.0196 & 0.1054 & 0.0028 & 1759 & 60 & 1790 & 96 & 1722 & 49 & -0.75 \\
\hline 103 & 19 & 17,782 & 4.771 & 0.342 & 0.3257 & 0.0199 & 0.1063 & 0.0028 & 1780 & 60 & 1817 & 97 & 1736 & 48 & -0.87 \\
\hline
\end{tabular}


Isotopic ratios (corrected)

Ages (Ma)

\begin{tabular}{|c|c|c|c|c|c|c|c|c|c|c|c|c|c|c|c|}
\hline \# & $\mathrm{N}$ & $\frac{{ }^{206} \mathrm{~Pb}}{{ }^{204} \mathrm{~Pb}^{\dagger}}$ & $\begin{array}{c}{ }^{207} \mathrm{~Pb}^{*} \\
{ }^{235} \mathrm{U}\end{array}$ & $\begin{array}{c} \pm \\
(\mathrm{abs})\end{array}$ & $\begin{array}{c}{ }^{206} \mathrm{~Pb}^{*} \\
{ }^{238} \mathrm{U}\end{array}$ & $\begin{array}{c} \pm \\
\text { (abs) }\end{array}$ & $\begin{array}{l}{ }^{207} \mathrm{~Pb}^{*} \\
{ }^{206} \mathrm{~Pb}^{*}\end{array}$ & $\begin{array}{c} \pm \\
(\mathrm{abs})\end{array}$ & $\begin{array}{c}{ }^{207} \mathrm{~Pb}^{*} \\
{ }^{235} \mathrm{U}\end{array}$ & $\begin{array}{c} \pm \\
\text { (abs) }\end{array}$ & $\begin{array}{c}{ }^{206} \mathrm{~Pb}^{*} \\
{ }^{238} \mathrm{U}\end{array}$ & $\begin{array}{c} \pm \\
\text { (abs) }\end{array}$ & $\begin{array}{l}{ }^{207} \mathrm{~Pb}^{*} \\
{ }^{206} \mathrm{~Pb}^{*}\end{array}$ & $\begin{array}{c} \pm \\
\text { (abs) }\end{array}$ & $\begin{array}{c}\text { Disc. } \\
(\%)\end{array}$ \\
\hline 104 & 16 & 4,994 & 4.657 & 0.377 & 0.3144 & 0.0220 & 0.1074 & 0.0029 & 1760 & 68 & 1762 & 108 & 1756 & 50 & -0.07 \\
\hline 105 & 14 & 6,093 & 6.798 & 0.799 & 0.4446 & 0.0471 & 0.1109 & 0.0031 & 2085 & 104 & 2371 & 210 & 1814 & 51 & -2.90 \\
\hline 106 & 10 & 1,768 & 3.989 & 0.349 & 0.2708 & 0.0191 & 0.1068 & 0.0036 & 1632 & 71 & 1545 & 97 & 1746 & 61 & 3.18 \\
\hline 107 & 20 & 15,985 & 4.466 & 0.308 & 0.3058 & 0.0178 & 0.1059 & 0.0028 & 1725 & 57 & 1720 & 88 & 1731 & 48 & 0.13 \\
\hline 109 & 18 & 15,955 & 4.778 & 0.353 & 0.3269 & 0.0207 & 0.1060 & 0.0028 & 1781 & 62 & 1824 & 101 & 1732 & 49 & -0.97 \\
\hline 110 & 16 & 14,987 & 5.985 & 0.511 & 0.3668 & 0.0275 & 0.1183 & 0.0032 & 1974 & 74 & 2015 & 130 & 1931 & 48 & -0.72 \\
\hline
\end{tabular}

Sample Q41: Quartzite, Lower Q4 unit, Uncompahgre Formation, Needle Mountains, Colorado

\begin{tabular}{|c|c|c|c|c|c|c|c|c|c|c|c|c|c|c|c|}
\hline 1 & 19 & 10,748 & 4.733 & 0.340 & 0.3128 & 0.0176 & 0.1098 & 0.0038 & 1773 & 60 & 1754 & 87 & 1795 & 63 & 0.49 \\
\hline 2 & 12 & 5,222 & 4.523 & 0.333 & 0.3170 & 0.0199 & 0.1035 & 0.0030 & 1735 & 61 & 1775 & 97 & 1687 & 53 & -0.98 \\
\hline 3 & 20 & 8,036 & 4.788 & 0.310 & 0.3198 & 0.0174 & 0.1086 & 0.0029 & 1783 & 54 & 1789 & 85 & 1776 & 49 & -0.15 \\
\hline 4 & 18 & 5,703 & 5.054 & 0.344 & 0.3468 & 0.0199 & 0.1057 & 0.0028 & 1828 & 58 & 1919 & 95 & 1726 & 49 & -1.79 \\
\hline 5 & 20 & 23,877 & 4.434 & 0.280 & 0.2989 & 0.0160 & 0.1076 & 0.0028 & 1719 & 52 & 1686 & 80 & 1759 & 48 & 0.95 \\
\hline 6 & 19 & 18,614 & 5.674 & 0.398 & 0.3775 & 0.0227 & 0.1090 & 0.0029 & 1927 & 61 & 2065 & 106 & 1783 & 48 & -2.18 \\
\hline 7 & 20 & 7,232 & 5.256 & 0.374 & 0.3546 & 0.0218 & 0.1075 & 0.0028 & 1862 & 61 & 1956 & 104 & 1758 & 48 & -1.76 \\
\hline 8 & 20 & 6,456 & 5.889 & 0.397 & 0.3623 & 0.0206 & 0.1179 & 0.0031 & 1960 & 59 & 1993 & 98 & 1925 & 47 & -0.61 \\
\hline 9 & 19 & 12,746 & 4.672 & 0.303 & 0.3176 & 0.0174 & 0.1067 & 0.0028 & 1762 & 54 & 1778 & 85 & 1744 & 48 & -0.38 \\
\hline 10 & 19 & 16,895 & 4.466 & 0.283 & 0.2975 & 0.0160 & 0.1089 & 0.0029 & 1725 & 53 & 1679 & 79 & 1781 & 48 & 1.34 \\
\hline 11 & 15 & 9,739 & 4.360 & 0.280 & 0.2831 & 0.0153 & 0.1117 & 0.0030 & 1705 & 53 & 1607 & 77 & 1827 & 49 & 3.27 \\
\hline 12 & 20 & 6,696 & 4.040 & 0.269 & 0.2774 & 0.0159 & 0.1056 & 0.0028 & 1642 & 54 & 1578 & 80 & 1725 & 48 & 2.19 \\
\hline 13 & 18 & 8,462 & 4.062 & 0.256 & 0.2826 & 0.0150 & 0.1042 & 0.0028 & 1647 & 51 & 1604 & 75 & 1701 & 49 & 1.38 \\
\hline 14 & 10 & 10,243 & 4.289 & 0.395 & 0.2948 & 0.0248 & 0.1055 & 0.0030 & 1691 & 76 & 1665 & 124 & 1723 & 52 & 0.76 \\
\hline 15 & 19 & 9,912 & 4.293 & 0.295 & 0.2886 & 0.0173 & 0.1079 & 0.0028 & 1692 & 57 & 1635 & 86 & 1764 & 48 & 1.80 \\
\hline 16 & 20 & 4,704 & 4.455 & 0.295 & 0.3038 & 0.0168 & 0.1063 & 0.0029 & 1723 & 55 & 1710 & 83 & 1737 & 50 & 0.34 \\
\hline 17 & 18 & 13,827 & 4.208 & 0.267 & 0.2935 & 0.0158 & 0.1040 & 0.0027 & 1676 & 52 & 1659 & 79 & 1697 & 49 & 0.50 \\
\hline 18 & 19 & 7,566 & 4.360 & 0.277 & 0.2999 & 0.0161 & 0.1054 & 0.0028 & 1705 & 53 & 1691 & 80 & 1722 & 48 & 0.40 \\
\hline 19 & 18 & 4,067 & 4.921 & 0.324 & 0.3175 & 0.0174 & 0.1124 & 0.0030 & 1806 & 55 & 1777 & 85 & 1839 & 49 & 0.72 \\
\hline 20 & 18 & 7,926 & 4.444 & 0.289 & 0.3056 & 0.0169 & 0.1055 & 0.0028 & 1720 & 54 & 1719 & 83 & 1722 & 49 & 0.04 \\
\hline 21 & 10 & 327 & 4.965 & 0.571 & 0.2733 & 0.0248 & 0.1317 & 0.0049 & 1813 & 97 & 1558 & 125 & 2121 & 65 & 9.97 \\
\hline 22 & 9 & 2,948 & 4.439 & 0.329 & 0.3021 & 0.0187 & 0.1066 & 0.0032 & 1720 & 61 & 1702 & 92 & 1741 & 55 & 0.50 \\
\hline 23 & 19 & 11,289 & 4.672 & 0.300 & 0.3090 & 0.0168 & 0.1096 & 0.0029 & 1762 & 54 & 1736 & 83 & 1793 & 48 & 0.70 \\
\hline 24 & 13 & 2,985 & 2.617 & 0.219 & 0.1754 & 0.0133 & 0.1082 & 0.0030 & 1305 & 62 & 1042 & 73 & 1769 & 51 & 25.46 \\
\hline 25 & 19 & 11,317 & 4.281 & 0.269 & 0.2851 & 0.0151 & 0.1089 & 0.0029 & 1690 & 52 & 1617 & 76 & 1781 & 48 & 2.37 \\
\hline 26 & 19 & 18,692 & 4.721 & 0.307 & 0.3085 & 0.0171 & 0.1110 & 0.0029 & 1771 & 54 & 1733 & 84 & 1816 & 48 & 1.02 \\
\hline 27 & 19 & 7,525 & 11.363 & 0.839 & 0.4419 & 0.0277 & 0.1865 & 0.0049 & 2553 & 69 & 2359 & 124 & 2711 & 43 & 2.53 \\
\hline 28 & 19 & 7,109 & 4.380 & 0.281 & 0.2993 & 0.0162 & 0.1062 & 0.0028 & 1709 & 53 & 1688 & 80 & 1734 & 48 & 0.60 \\
\hline 29 & 18 & 4,925 & 5.125 & 0.387 & 0.3488 & 0.0229 & 0.1066 & 0.0029 & 1840 & 64 & 1929 & 109 & 1742 & 50 & -1.72 \\
\hline 30 & 18 & 9,084 & 4.845 & 0.322 & 0.3232 & 0.0183 & 0.1087 & 0.0029 & 1793 & 56 & 1805 & 89 & 1778 & 48 & -0.30 \\
\hline 31 & 17 & 3,606 & 4.777 & 0.329 & 0.3259 & 0.0190 & 0.1063 & 0.0029 & 1781 & 58 & 1819 & 92 & 1737 & 50 & -0.87 \\
\hline 32 & 17 & 3,555 & 5.430 & 0.419 & 0.3660 & 0.0244 & 0.1076 & 0.0029 & 1890 & 66 & 2010 & 115 & 1759 & 50 & -2.07 \\
\hline 33 & 15 & 7,569 & 5.311 & 0.397 & 0.3593 & 0.0233 & 0.1072 & 0.0029 & 1871 & 64 & 1979 & 110 & 1753 & 50 & -1.94 \\
\hline 34 & 18 & 10,133 & 4.935 & 0.332 & 0.3204 & 0.0184 & 0.1117 & 0.0030 & 1808 & 57 & 1792 & 90 & 1827 & 48 & 0.41 \\
\hline 35 & 18 & 7,363 & 5.618 & 0.398 & 0.3631 & 0.0221 & 0.1122 & 0.0030 & 1919 & 61 & 1997 & 104 & 1835 & 48 & -1.39 \\
\hline 36 & 19 & 9,509 & 4.839 & 0.331 & 0.3293 & 0.0194 & 0.1066 & 0.0028 & 1792 & 58 & 1835 & 94 & 1741 & 48 & -0.98 \\
\hline 37 & 19 & 19,815 & 4.047 & 0.251 & 0.2745 & 0.0144 & 0.1069 & 0.0028 & 1644 & 50 & 1564 & 73 & 1747 & 48 & 2.82 \\
\hline
\end{tabular}


Isotopic ratios (corrected)

Ages (Ma)

\begin{tabular}{|c|c|c|c|c|c|c|c|c|c|c|c|c|c|c|c|}
\hline \# & $\mathrm{N}$ & ${ }^{206} \mathrm{~Pb}$ & $\begin{array}{c}{ }^{207} \mathrm{~Pb}^{*} \\
{ }^{235} \mathrm{U} \\
\end{array}$ & $\begin{array}{c} \pm \\
\text { (abs) }\end{array}$ & $\begin{array}{c}{ }^{206} \mathrm{~Pb}^{*} \\
{ }^{238} \mathrm{U} \\
\end{array}$ & $\begin{array}{c} \pm \\
(\mathrm{abs})\end{array}$ & $\begin{array}{l}{ }^{207} \mathrm{~Pb}^{*} \\
{ }^{206} \mathrm{~Pb}^{*}\end{array}$ & $\begin{array}{c} \pm \\
(\mathrm{abs})\end{array}$ & $\begin{array}{c}{ }^{207} \mathrm{~Pb}^{*} \\
{ }^{235} \mathrm{U} \\
\end{array}$ & $\begin{array}{c} \pm \\
\text { (abs) }\end{array}$ & $\begin{array}{c}{ }^{206} \mathrm{~Pb}^{*} \\
{ }^{238} \mathrm{U} \\
\end{array}$ & $\begin{array}{c} \pm \\
\text { (abs) }\end{array}$ & $\begin{array}{l}{ }^{207} \mathrm{~Pb}^{*} \\
{ }^{206} \mathrm{~Pb}^{*} \\
\end{array}$ & $\begin{array}{c} \pm \\
(\mathrm{abs})\end{array}$ & $\begin{array}{c}\text { Disc. } \\
(\%)\end{array}$ \\
\hline 38 & 16 & 10,773 & 4.028 & 0.256 & 0.2792 & 0.0150 & 0.1047 & 0.0028 & 1640 & 52 & 1587 & 75 & 1708 & 50 & 1.78 \\
\hline 39 & 14 & 5,197 & 4.650 & 0.319 & 0.3076 & 0.0178 & 0.1096 & 0.0031 & 1758 & 57 & 1729 & 88 & 1793 & 51 & 0.79 \\
\hline 40 & 19 & 13,246 & 4.538 & 0.285 & 0.2928 & 0.0156 & 0.1124 & 0.0030 & 1738 & 52 & 1656 & 78 & 1838 & 48 & 2.54 \\
\hline 41 & 17 & 4,807 & 5.005 & 0.421 & 0.3299 & 0.0249 & 0.1100 & 0.0030 & 1820 & 71 & 1838 & 121 & 1800 & 49 & -0.41 \\
\hline 42 & 18 & 6,597 & 5.913 & 0.449 & 0.3903 & 0.0256 & 0.1099 & 0.0030 & 1963 & 66 & 2124 & 119 & 1797 & 49 & -2.35 \\
\hline 43 & 20 & 5,382 & 4.890 & 0.324 & 0.3325 & 0.0185 & 0.1067 & 0.0028 & 1801 & 56 & 1850 & 90 & 1743 & 49 & -1.10 \\
\hline 44 & 19 & 4,898 & 7.672 & 0.524 & 0.3164 & 0.0187 & 0.1759 & 0.0046 & 2193 & 61 & 1772 & 91 & 2614 & 44 & 13.09 \\
\hline 45 & 19 & 6,071 & 4.531 & 0.297 & 0.3127 & 0.0173 & 0.1051 & 0.0028 & 1737 & 55 & 1754 & 85 & 1716 & 49 & -0.44 \\
\hline 46 & 20 & 5,676 & 4.133 & 0.264 & 0.2870 & 0.0153 & 0.1044 & 0.0029 & 1661 & 52 & 1626 & 76 & 1705 & 50 & 1.08 \\
\hline 47 & 20 & 10,043 & 4.429 & 0.286 & 0.3046 & 0.0167 & 0.1054 & 0.0028 & 1718 & 53 & 1714 & 83 & 1722 & 48 & 0.10 \\
\hline 48 & 19 & 23,927 & 4.341 & 0.277 & 0.2957 & 0.0161 & 0.1065 & 0.0028 & 1701 & 53 & 1670 & 80 & 1740 & 48 & 0.91 \\
\hline 49 & 20 & 2,990 & 3.215 & 0.199 & 0.2182 & 0.0114 & 0.1069 & 0.0029 & 1461 & 48 & 1272 & 60 & 1747 & 49 & 11.31 \\
\hline 50 & 20 & 22,774 & 8.736 & 0.601 & 0.3913 & 0.0229 & 0.1619 & 0.0042 & 2311 & 63 & 2129 & 106 & 2476 & 44 & 3.12 \\
\hline 51 & 13 & 3,843 & 3.898 & 0.315 & 0.2655 & 0.0191 & 0.1065 & 0.0030 & 1613 & 65 & 1518 & 97 & 1740 & 52 & 3.64 \\
\hline 52 & 19 & 7,965 & 13.426 & 1.462 & 0.5391 & 0.0517 & 0.1806 & 0.0047 & 2710 & 103 & 2780 & 216 & 2659 & 43 & -0.49 \\
\hline 53 & 11 & 3,394 & 6.376 & 0.797 & 0.4322 & 0.0499 & 0.1070 & 0.0031 & 2029 & 110 & 2316 & 225 & 1749 & 53 & -3.11 \\
\hline 54 & 15 & 3,187 & 5.088 & 0.368 & 0.3437 & 0.0211 & 0.1074 & 0.0029 & 1834 & 61 & 1905 & 101 & 1755 & 50 & -1.42 \\
\hline 55 & 17 & 4,868 & 6.371 & 0.620 & 0.4304 & 0.0378 & 0.1074 & 0.0029 & 2028 & 85 & 2307 & 171 & 1755 & 49 & -3.07 \\
\hline 56 & 14 & 1,467 & 6.821 & 0.613 & 0.4416 & 0.0336 & 0.1120 & 0.0032 & 2088 & 80 & 2358 & 150 & 1832 & 51 & -2.80 \\
\hline 57 & 20 & 5,406 & 5.038 & 0.362 & 0.3390 & 0.0211 & 0.1078 & 0.0028 & 1826 & 61 & 1882 & 102 & 1762 & 48 & -1.18 \\
\hline 58 & 19 & 8,632 & 4.068 & 0.258 & 0.2758 & 0.0148 & 0.1070 & 0.0028 & 1648 & 52 & 1570 & 75 & 1748 & 48 & 2.71 \\
\hline 59 & 20 & 14,322 & 4.865 & 0.313 & 0.3164 & 0.0172 & 0.1115 & 0.0029 & 1796 & 54 & 1772 & 84 & 1824 & 48 & 0.61 \\
\hline 60 & 19 & 7,605 & 5.046 & 0.352 & 0.3427 & 0.0205 & 0.1068 & 0.0028 & 1827 & 59 & 1900 & 98 & 1745 & 49 & -1.48 \\
\hline 61 & 11 & 1,174 & 2.474 & 0.246 & 0.1547 & 0.0139 & 0.1159 & 0.0034 & 1264 & 72 & 927 & 77 & 1895 & 53 & -36.32 \\
\hline 62 & 12 & 2,480 & 4.462 & 0.303 & 0.2953 & 0.0166 & 0.1096 & 0.0031 & 1724 & 56 & 1668 & 82 & 1792 & 52 & 1.67 \\
\hline 63 & 19 & 19,900 & 4.935 & 0.322 & 0.3332 & 0.0185 & 0.1074 & 0.0028 & 1808 & 55 & 1854 & 89 & 1756 & 48 & -1.00 \\
\hline 64 & 19 & 4,277 & 4.529 & 0.293 & 0.3048 & 0.0165 & 0.1077 & 0.0029 & 1736 & 54 & 1715 & 82 & 1762 & 49 & 0.58 \\
\hline 65 & 19 & 41,199 & 10.201 & 0.715 & 0.4070 & 0.0242 & 0.1818 & 0.0047 & 2453 & 65 & 2201 & 111 & 2669 & 43 & 4.10 \\
\hline 66 & 14 & 4,865 & 4.379 & 0.290 & 0.2952 & 0.0163 & 0.1076 & 0.0030 & 1708 & 55 & 1668 & 81 & 1759 & 51 & 1.21 \\
\hline 67 & 19 & 11,951 & 5.116 & 0.346 & 0.3546 & 0.0203 & 0.1047 & 0.0028 & 1839 & 57 & 1956 & 97 & 1708 & 48 & -2.17 \\
\hline 68 & 19 & 2,695 & 3.264 & 0.201 & 0.2208 & 0.0114 & 0.1072 & 0.0029 & 1472 & 48 & 1286 & 60 & 1752 & 49 & 10.87 \\
\hline 69 & 7 & 12,336 & 6.536 & 1.024 & 0.3587 & 0.0538 & 0.1321 & 0.0043 & 2051 & 138 & 1976 & 255 & 2127 & 57 & 1.48 \\
\hline 70 & 20 & 3,910 & 4.393 & 0.282 & 0.2991 & 0.0160 & 0.1065 & 0.0028 & 1711 & 53 & 1687 & 80 & 1741 & 49 & 0.70 \\
\hline 71 & 19 & 10,079 & 4.784 & 0.316 & 0.3238 & 0.0182 & 0.1072 & 0.0028 & 1782 & 56 & 1808 & 89 & 1752 & 48 & -0.62 \\
\hline 72 & 9 & 3,527 & 6.375 & 0.599 & 0.4325 & 0.0354 & 0.1069 & 0.0032 & 2029 & 82 & 2317 & 159 & 1748 & 54 & -3.11 \\
\hline 73 & 20 & 9,119 & 3.990 & 0.248 & 0.2759 & 0.0145 & 0.1049 & 0.0028 & 1632 & 51 & 1570 & 73 & 1712 & 49 & 2.13 \\
\hline 74 & 20 & 4,274 & 4.941 & 0.340 & 0.3415 & 0.0199 & 0.1049 & 0.0028 & 1809 & 58 & 1894 & 96 & 1713 & 49 & -1.72 \\
\hline 75 & 20 & 14,409 & 5.022 & 0.333 & 0.3409 & 0.0192 & 0.1068 & 0.0028 & 1823 & 56 & 1891 & 92 & 1746 & 48 & -1.40 \\
\hline 76 & 19 & 12,592 & 5.119 & 0.340 & 0.3411 & 0.0192 & 0.1088 & 0.0029 & 1839 & 56 & 1892 & 92 & 1780 & 48 & -1.09 \\
\hline 77 & 20 & 3,861 & 4.543 & 0.309 & 0.3136 & 0.0180 & 0.1051 & 0.0029 & 1739 & 57 & 1758 & 88 & 1716 & 50 & -0.49 \\
\hline 78 & 20 & 4,386 & 4.603 & 0.312 & 0.3178 & 0.0182 & 0.1051 & 0.0028 & 1750 & 57 & 1779 & 89 & 1715 & 49 & -0.71 \\
\hline 79 & 18 & 3,553 & 3.998 & 0.267 & 0.2650 & 0.0150 & 0.1094 & 0.0030 & 1634 & 54 & 1516 & 77 & 1789 & 50 & 4.58 \\
\hline 80 & 19 & 5,568 & 4.781 & 0.317 & 0.3137 & 0.0177 & 0.1106 & 0.0029 & 1782 & 56 & 1759 & 87 & 1809 & 48 & 0.59 \\
\hline 81 & 15 & 7,149 & 4.344 & 0.285 & 0.2977 & 0.0165 & 0.1058 & 0.0029 & 1702 & 54 & 1680 & 82 & 1729 & 50 & 0.63 \\
\hline 82 & 20 & 5,050 & 4.743 & 0.322 & 0.3321 & 0.0189 & 0.1036 & 0.0028 & 1775 & 57 & 1849 & 92 & 1689 & 50 & -1.61 \\
\hline 83 & 18 & 14,958 & 4.371 & 0.281 & 0.2985 & 0.0163 & 0.1062 & 0.0028 & 1707 & 53 & 1684 & 81 & 1735 & 49 & 0.67 \\
\hline
\end{tabular}


Isotopic ratios (corrected)

Ages (Ma)

\begin{tabular}{|c|c|c|c|c|c|c|c|c|c|c|c|c|c|c|c|}
\hline \# & $\mathrm{N}$ & $\frac{{ }^{206} \mathrm{~Pb}}{{ }^{204} \mathrm{~Pb}^{\dagger}}$ & $\begin{array}{c}{ }^{207} \mathrm{~Pb}^{*} \\
{ }^{235} \mathrm{U} \\
\end{array}$ & $\begin{array}{c} \pm \\
(\mathrm{abs})\end{array}$ & $\begin{array}{c}{ }^{206} \mathrm{~Pb}^{*} \\
{ }^{238} \mathrm{U} \\
\end{array}$ & $\begin{array}{c} \pm \\
\text { (abs) }\end{array}$ & $\begin{array}{l}{ }^{207} \mathrm{~Pb}^{*} \\
{ }^{206} \mathrm{~Pb}^{*} \\
\end{array}$ & $\begin{array}{c} \pm \\
\text { (abs) }\end{array}$ & $\begin{array}{c}{ }^{207} \mathrm{~Pb}^{*} \\
{ }^{235} \mathrm{U} \\
\end{array}$ & $\begin{array}{c} \pm \\
(\mathrm{abs}) \\
\end{array}$ & $\begin{array}{c}{ }^{206} \mathrm{~Pb}^{*} \\
{ }^{238} \mathrm{U} \\
\end{array}$ & $\begin{array}{c} \pm \\
(\mathrm{abs}) \\
\end{array}$ & $\begin{array}{l}{ }^{207} \mathrm{~Pb}^{*} \\
{ }^{206} \mathrm{~Pb}^{*}\end{array}$ & $\begin{array}{c} \pm \\
\text { (abs) }\end{array}$ & $\begin{array}{c}\text { Disc. } \\
\text { (\%) }\end{array}$ \\
\hline 84 & 20 & 10,334 & 4.453 & 0.284 & 0.3081 & 0.0167 & 0.1048 & 0.0027 & 1722 & 53 & 1732 & 82 & 1711 & 48 & -0.24 \\
\hline 85 & 17 & 8,821 & 4.512 & 0.293 & 0.3104 & 0.0170 & 0.1054 & 0.0028 & 1733 & 54 & 1743 & 84 & 1722 & 49 & -0.24 \\
\hline 86 & 19 & 6,524 & 4.852 & 0.320 & 0.3315 & 0.0184 & 0.1062 & 0.0028 & 1794 & 56 & 1846 & 89 & 1734 & 49 & -1.14 \\
\hline 87 & 19 & 13,438 & 4.409 & 0.280 & 0.3025 & 0.0163 & 0.1057 & 0.0028 & 1714 & 53 & 1704 & 81 & 1727 & 48 & 0.28 \\
\hline 89 & 18 & 13,052 & 5.074 & 0.335 & 0.3374 & 0.0189 & 0.1091 & 0.0029 & 1832 & 56 & 1874 & 91 & 1784 & 48 & -0.91 \\
\hline 90 & 12 & 2,041 & 5.426 & 0.415 & 0.3583 & 0.0228 & 0.1098 & 0.0032 & 1889 & 66 & 1974 & 108 & 1797 & 53 & -1.56 \\
\hline 91 & 11 & 2,218 & 4.494 & 0.359 & 0.3016 & 0.0207 & 0.1080 & 0.0032 & 1730 & 66 & 1699 & 102 & 1767 & 54 & 0.86 \\
\hline 92 & 15 & 1,572 & 4.933 & 0.357 & 0.3275 & 0.0197 & 0.1093 & 0.0030 & 1808 & 61 & 1826 & 96 & 1787 & 51 & -0.42 \\
\hline 93 & 19 & 5,048 & 4.155 & 0.267 & 0.2866 & 0.0156 & 0.1052 & 0.0028 & 1665 & 53 & 1624 & 78 & 1717 & 49 & 1.29 \\
\hline 98 & 19 & 12,049 & 4.609 & 0.304 & 0.3181 & 0.0179 & 0.1051 & 0.0028 & 1751 & 55 & 1780 & 88 & 1716 & 48 & -0.72 \\
\hline 99 & 19 & 6,656 & 4.690 & 0.308 & 0.3242 & 0.0180 & 0.1049 & 0.0028 & 1765 & 55 & 1810 & 88 & 1713 & 49 & -1.04 \\
\hline 100 & 18 & 11,082 & 4.303 & 0.278 & 0.2982 & 0.0164 & 0.1047 & 0.0028 & 1694 & 53 & 1682 & 81 & 1708 & 49 & 0.34 \\
\hline 101 & 20 & 5,138 & 4.696 & 0.313 & 0.3267 & 0.0183 & 0.1042 & 0.0028 & 1767 & 56 & 1822 & 89 & 1701 & 49 & -1.27 \\
\hline 102 & 20 & 14,619 & 4.917 & 0.327 & 0.3283 & 0.0187 & 0.1086 & 0.0028 & 1805 & 56 & 1830 & 91 & 1777 & 48 & -0.57 \\
\hline
\end{tabular}

\begin{tabular}{|c|c|c|c|c|c|c|c|c|c|c|c|c|c|c|}
\hline \multicolumn{15}{|c|}{ Sample J03-NM4: Basal conglomerate, Uncompahgre Formation, Needle Mountains, Colorado } \\
\hline 1 & 46,844 & 4.372 & 0.183 & 0.2969 & 0.0124 & 0.1068 & 0.0027 & 1707 & 35 & 1676 & 62 & 1745 & 46 & 0.91 \\
\hline 2 & 22,883 & 4.355 & 0.184 & 0.2924 & 0.0122 & 0.1080 & 0.0028 & 1704 & 35 & 1653 & 61 & 1767 & 47 & 1.54 \\
\hline 4 & 17,276 & 4.315 & 0.179 & 0.2885 & 0.0120 & 0.1085 & 0.0027 & 1696 & 34 & 1634 & 60 & 1774 & 45 & 1.95 \\
\hline 5 & 22,286 & 4.985 & 0.215 & 0.3362 & 0.0145 & 0.1075 & 0.0027 & 1817 & 36 & 1869 & 70 & 1758 & 45 & -1.11 \\
\hline 6 & 909 & 2.520 & 0.141 & 0.1420 & 0.0053 & 0.1287 & 0.0064 & 1278 & 41 & 856 & 30 & 2080 & 88 & 67.30 \\
\hline 7 & 4,999 & 4.157 & 0.150 & 0.2767 & 0.0098 & 0.1089 & 0.0029 & 1666 & 30 & 1575 & 49 & 1782 & 48 & 3.16 \\
\hline 8 & 9,606 & 4.199 & 0.174 & 0.2870 & 0.0119 & 0.1061 & 0.0027 & 1674 & 34 & 1626 & 60 & 1734 & 46 & 1.50 \\
\hline 9 & 2,436 & 4.086 & 0.172 & 0.2597 & 0.0106 & 0.1141 & 0.0031 & 1652 & 34 & 1488 & 54 & 1866 & 48 & 6.74 \\
\hline 10 & 15,427 & 4.575 & 0.194 & 0.3070 & 0.0129 & 0.1081 & 0.0027 & 1745 & 35 & 1726 & 64 & 1767 & 46 & 0.50 \\
\hline 11 & 752 & 5.955 & 0.260 & 0.3401 & 0.0147 & 0.1270 & 0.0032 & 1969 & 38 & 1887 & 71 & 2057 & 45 & 1.83 \\
\hline 14 & 13,475 & 4.337 & 0.183 & 0.2908 & 0.0121 & 0.1082 & 0.0028 & 1700 & 35 & 1645 & 60 & 1769 & 47 & 1.70 \\
\hline 15 & 4,420 & 4.241 & 0.175 & 0.2817 & 0.0116 & 0.1092 & 0.0027 & 1682 & 34 & 1600 & 59 & 1786 & 46 & 2.75 \\
\hline 16 & 39,647 & 4.013 & 0.165 & 0.2746 & 0.0113 & 0.1060 & 0.0026 & 1637 & 33 & 1564 & 57 & 1731 & 46 & 2.55 \\
\hline 17 & 15,376 & 3.958 & 0.162 & 0.2705 & 0.0111 & 0.1061 & 0.0026 & 1626 & 33 & 1543 & 56 & 1734 & 46 & 3.00 \\
\hline 18 & 13,752 & 3.982 & 0.162 & 0.2676 & 0.0110 & 0.1079 & 0.0027 & 1631 & 33 & 1529 & 56 & 1764 & 45 & 3.83 \\
\hline 19 & 17,586 & 3.800 & 0.139 & 0.2545 & 0.0092 & 0.1083 & 0.0028 & 1593 & 29 & 1462 & 47 & 1771 & 47 & 5.55 \\
\hline 21 & 6,487 & 3.954 & 0.165 & 0.2619 & 0.0107 & 0.1095 & 0.0029 & 1625 & 34 & 1500 & 55 & 1791 & 48 & 4.98 \\
\hline 22 & 38,519 & 3.976 & 0.163 & 0.2727 & 0.0112 & 0.1057 & 0.0026 & 1629 & 33 & 1555 & 57 & 1727 & 45 & 2.66 \\
\hline 23 & 35,973 & 4.198 & 0.174 & 0.2874 & 0.0119 & 0.1060 & 0.0026 & 1674 & 34 & 1628 & 60 & 1731 & 46 & 1.43 \\
\hline 24 & 2,001 & 5.256 & 0.235 & 0.3283 & 0.0141 & 0.1161 & 0.0032 & 1862 & 38 & 1830 & 68 & 1897 & 49 & 0.75 \\
\hline
\end{tabular}


Isotopic ratios (corrected)

Ages (Ma)

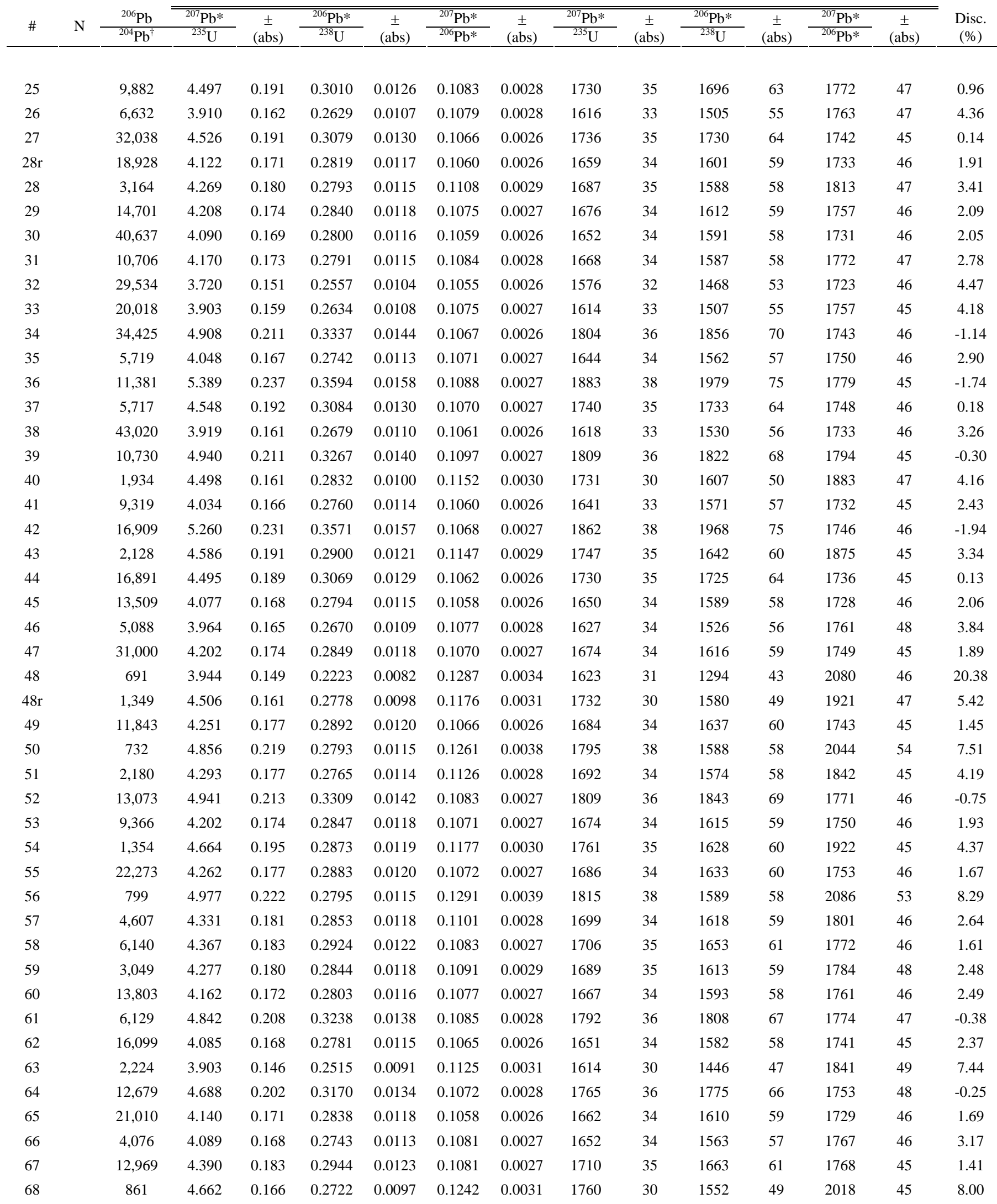


Isotopic ratios (corrected)

Ages (Ma)

\begin{tabular}{|c|c|c|c|c|c|c|c|c|c|c|c|c|c|c|c|}
\hline$\#$ & $\mathrm{~N}$ & $\frac{{ }^{206} \mathrm{~Pb}}{{ }^{204} \mathrm{~Pb}^{\dagger}}$ & $\begin{array}{c}{ }^{207} \mathrm{~Pb}^{*} \\
{ }^{235} \mathrm{U}\end{array}$ & $\begin{array}{c} \pm \\
\text { (abs) }\end{array}$ & $\begin{array}{l}{ }^{206} \mathrm{~Pb}^{*} \\
{ }^{238} \mathrm{U}\end{array}$ & $\begin{array}{c} \pm \\
(\mathrm{abs})\end{array}$ & $\begin{array}{l}{ }^{207} \mathrm{~Pb}^{*} \\
{ }^{206} \mathrm{~Pb}^{*}\end{array}$ & $\begin{array}{c} \pm \\
(\mathrm{abs})\end{array}$ & $\begin{array}{c}{ }^{207} \mathrm{~Pb}^{*} \\
{ }^{235} \mathrm{U}\end{array}$ & $\stackrel{ \pm}{ \pm}$ & $\begin{array}{l}{ }^{206} \mathrm{~Pb}^{*} \\
{ }^{238} \mathrm{U}\end{array}$ & $\begin{array}{c} \pm \\
(\mathrm{abs})\end{array}$ & $\begin{array}{l}{ }^{207} \mathrm{~Pb}^{*} \\
{ }^{206} \mathrm{~Pb}^{*}\end{array}$ & $\begin{array}{c} \pm \\
\text { (abs) }\end{array}$ & $\begin{array}{c}\text { Disc. } \\
(\%)\end{array}$ \\
\hline 69 & & 10,236 & 4.424 & 0.185 & 0.2944 & 0.0123 & 0.1090 & 0.0027 & 1717 & 35 & 1663 & 61 & 1783 & 46 & 1.61 \\
\hline 70 & & 13,660 & 4.290 & 0.178 & 0.2879 & 0.0120 & 0.1081 & 0.0027 & 1691 & 34 & 1631 & 60 & 1767 & 46 & 1.90 \\
\hline 71 & & 1,479 & 4.513 & 0.187 & 0.2767 & 0.0114 & 0.1183 & 0.0030 & 1733 & 34 & 1574 & 58 & 1931 & 46 & 5.74 \\
\hline 72 & & 11,078 & 3.872 & 0.158 & 0.2611 & 0.0106 & 0.1075 & 0.0027 & 1608 & 33 & 1496 & 54 & 1758 & 46 & 4.47 \\
\hline 74 & & 5,712 & 4.085 & 0.169 & 0.2712 & 0.0111 & 0.1093 & 0.0028 & 1651 & 34 & 1547 & 56 & 1787 & 46 & 3.83 \\
\hline 75 & & 7,797 & 3.992 & 0.164 & 0.2691 & 0.0110 & 0.1076 & 0.0027 & 1633 & 33 & 1536 & 56 & 1759 & 46 & 3.58 \\
\hline 76 & & 3,057 & 4.403 & 0.156 & 0.2871 & 0.0101 & 0.1112 & 0.0028 & 1713 & 29 & 1627 & 50 & 1819 & 46 & 2.76 \\
\hline $76 r$ & & 2,403 & 4.100 & 0.168 & 0.2652 & 0.0108 & 0.1121 & 0.0028 & 1654 & 33 & 1516 & 55 & 1834 & 45 & 5.39 \\
\hline 77 & & 13,626 & 4.522 & 0.192 & 0.3010 & 0.0126 & 0.1089 & 0.0029 & 1735 & 35 & 1696 & 62 & 1782 & 48 & 1.10 \\
\hline 81 & & 2,968 & 5.188 & 0.224 & 0.3332 & 0.0144 & 0.1129 & 0.0028 & 1851 & 37 & 1854 & 69 & 1847 & 45 & -0.07 \\
\hline 82 & & 4,914 & 4.616 & 0.195 & 0.3053 & 0.0129 & 0.1096 & 0.0028 & 1752 & 35 & 1718 & 63 & 1794 & 46 & 0.95 \\
\hline 83 & & 3,647 & 5.077 & 0.225 & 0.3265 & 0.0140 & 0.1128 & 0.0031 & 1832 & 38 & 1821 & 68 & 1845 & 49 & 0.26 \\
\hline 84 & & 12,148 & 4.346 & 0.183 & 0.2923 & 0.0122 & 0.1078 & 0.0027 & 1702 & 35 & 1653 & 61 & 1763 & 46 & 1.49 \\
\hline 85 & & 3,796 & 4.656 & 0.199 & 0.3101 & 0.0131 & 0.1089 & 0.0028 & 1759 & 36 & 1741 & 64 & 1781 & 47 & 0.48 \\
\hline 86 & & 10,828 & 4.173 & 0.172 & 0.2812 & 0.0116 & 0.1076 & 0.0027 & 1669 & 34 & 1597 & 58 & 1759 & 46 & 2.38 \\
\hline 87 & & 1,275 & 4.231 & 0.192 & 0.2552 & 0.0104 & 0.1202 & 0.0038 & 1680 & 37 & 1465 & 53 & 1960 & 56 & 9.45 \\
\hline 88 & & 17,498 & 4.013 & 0.164 & 0.2690 & 0.0110 & 0.1082 & 0.0027 & 1637 & 33 & 1536 & 56 & 1769 & 45 & 3.76 \\
\hline
\end{tabular}

\begin{tabular}{|c|c|c|c|c|c|c|c|c|c|c|c|c|c|c|c|}
\hline \multicolumn{16}{|c|}{ Sample J05-VC2: Fall Creek Formation, Vallecito Conglomerate, Needle Mountains, Colorado } \\
\hline 1 & 19 & 19,294 & 3.605 & 0.215 & 0.2425 & 0.0122 & 0.1078 & 0.0028 & 1551 & 47 & 1400 & 64 & 1763 & 48 & 7.14 \\
\hline 2 & 20 & 17,012 & 3.609 & 0.214 & 0.2440 & 0.0123 & 0.1073 & 0.0028 & 1552 & 47 & 1407 & 64 & 1754 & 48 & 6.72 \\
\hline 3 & 17 & 26,961 & 3.748 & 0.227 & 0.2532 & 0.0131 & 0.1073 & 0.0028 & 1582 & 49 & 1455 & 67 & 1755 & 48 & 5.41 \\
\hline 4 & 20 & 8,249 & 3.624 & 0.218 & 0.2473 & 0.0125 & 0.1063 & 0.0029 & 1555 & 48 & 1424 & 64 & 1737 & 50 & 5.88 \\
\hline 5 & 19 & 41,090 & 4.329 & 0.276 & 0.2937 & 0.0161 & 0.1069 & 0.0028 & 1699 & 53 & 1660 & 80 & 1747 & 48 & 1.16 \\
\hline 6 & 14 & 7,028 & 3.796 & 0.237 & 0.2582 & 0.0135 & 0.1066 & 0.0029 & 1592 & 50 & 1481 & 69 & 1743 & 50 & 4.54 \\
\hline 7 & 19 & 18,618 & 4.504 & 0.292 & 0.3056 & 0.0171 & 0.1069 & 0.0028 & 1732 & 54 & 1719 & 84 & 1747 & 48 & 0.34 \\
\hline 8 & 17 & 8,956 & 3.577 & 0.216 & 0.2419 & 0.0123 & 0.1073 & 0.0029 & 1544 & 48 & 1396 & 64 & 1753 & 50 & 7.04 \\
\hline 9 & 19 & 19,637 & 3.577 & 0.212 & 0.2426 & 0.0122 & 0.1069 & 0.0028 & 1544 & 47 & 1400 & 63 & 1748 & 48 & 6.79 \\
\hline 10 & 19 & 21,210 & 4.641 & 0.303 & 0.3149 & 0.0177 & 0.1069 & 0.0028 & 1757 & 55 & 1765 & 87 & 1747 & 48 & -0.21 \\
\hline 11 & 18 & 9,539 & 3.582 & 0.215 & 0.2436 & 0.0123 & 0.1067 & 0.0028 & 1545 & 48 & 1405 & 64 & 1743 & 49 & 6.55 \\
\hline 12 & 20 & 6,588 & 3.641 & 0.219 & 0.2473 & 0.0124 & 0.1068 & 0.0029 & 1559 & 48 & 1425 & 64 & 1745 & 50 & 6.03 \\
\hline 13 & 18 & 11,287 & 3.590 & 0.217 & 0.2438 & 0.0125 & 0.1068 & 0.0028 & 1547 & 48 & 1406 & 65 & 1746 & 49 & 6.57 \\
\hline 14 & 17 & 3,505 & 4.175 & 0.272 & 0.2829 & 0.0153 & 0.1070 & 0.0031 & 1669 & 53 & 1606 & 77 & 1749 & 52 & 2.07 \\
\hline 15 & 19 & 10,778 & 3.806 & 0.230 & 0.2588 & 0.0132 & 0.1067 & 0.0028 & 1594 & 49 & 1484 & 68 & 1743 & 48 & 4.47 \\
\hline 16 & 18 & 10,509 & 3.889 & 0.238 & 0.2620 & 0.0137 & 0.1076 & 0.0028 & 1611 & 50 & 1500 & 70 & 1760 & 48 & 4.39 \\
\hline 17 & 15 & 7,066 & 3.671 & 0.226 & 0.2517 & 0.0131 & 0.1058 & 0.0029 & 1565 & 49 & 1447 & 68 & 1728 & 50 & 5.07 \\
\hline 18 & 18 & 11,113 & 3.128 & 0.193 & 0.2479 & 0.0131 & 0.0915 & 0.0024 & 1439 & 48 & 1427 & 68 & 1457 & 50 & 0.51 \\
\hline 19 & 20 & 7,572 & 3.973 & 0.245 & 0.2715 & 0.0142 & 0.1061 & 0.0028 & 1629 & 50 & 1548 & 72 & 1734 & 49 & 2.90 \\
\hline 22 & 20 & 19,783 & 4.709 & 0.307 & 0.3205 & 0.0180 & 0.1066 & 0.0028 & 1769 & 55 & 1792 & 88 & 1742 & 48 & -0.56 \\
\hline 23 & 18 & 10,119 & 3.949 & 0.245 & 0.2688 & 0.0142 & 0.1066 & 0.0028 & 1624 & 50 & 1535 & 72 & 1741 & 49 & 3.30 \\
\hline 24 & 19 & 24,218 & 4.254 & 0.266 & 0.2884 & 0.0154 & 0.1070 & 0.0028 & 1684 & 51 & 1634 & 77 & 1748 & 48 & 1.60 \\
\hline 25 & 18 & 2,431 & 4.294 & 0.316 & 0.2887 & 0.0176 & 0.1079 & 0.0034 & 1692 & 61 & 1635 & 88 & 1764 & 58 & 1.79 \\
\hline
\end{tabular}


Isotopic ratios (corrected)

Ages (Ma)

\begin{tabular}{|c|c|c|c|c|c|c|c|c|c|c|c|c|c|c|c|}
\hline$\#$ & $\mathrm{~N}$ & $\frac{{ }^{206} \mathrm{~Pb}}{{ }^{204} \mathrm{~Pb}^{\dagger}}$ & $\begin{array}{l}{ }^{207} \mathrm{~Pb}^{*} \\
{ }^{235} \mathrm{U}\end{array}$ & $\begin{array}{c} \pm \\
\text { (abs) }\end{array}$ & $\begin{array}{l}{ }^{206} \mathrm{~Pb}^{*} \\
{ }^{238} \mathrm{U}\end{array}$ & $\begin{array}{c} \pm \\
\text { (abs) }\end{array}$ & $\begin{array}{l}{ }^{207} \mathrm{~Pb}^{*} \\
{ }^{206} \mathrm{~Pb}^{*}\end{array}$ & $\begin{array}{c} \pm \\
\text { (abs) }\end{array}$ & $\begin{array}{c}{ }^{207} \mathrm{~Pb}^{*} \\
{ }^{235} \mathrm{U}\end{array}$ & $\begin{array}{c} \pm \\
\text { (abs) }\end{array}$ & $\begin{array}{c}{ }^{206} \mathrm{~Pb}^{*} \\
{ }^{238} \mathrm{U}\end{array}$ & $\begin{array}{c} \pm \\
(\mathrm{abs})\end{array}$ & $\begin{array}{l}{ }^{207} \mathrm{~Pb}^{*} \\
{ }^{206} \mathrm{~Pb}^{*}\end{array}$ & $\begin{array}{c} \pm \\
(\mathrm{abs})\end{array}$ & $\begin{array}{c}\text { Disc. } \\
(\%)\end{array}$ \\
\hline 26 & 20 & 9,008 & 4.313 & 0.271 & 0.2937 & 0.0157 & 0.1065 & 0.0028 & 1696 & 52 & 1660 & 78 & 1740 & 49 & 1.07 \\
\hline 27 & 19 & 13,136 & 4.958 & 0.342 & 0.3356 & 0.0202 & 0.1071 & 0.0028 & 1812 & 58 & 1866 & 97 & 1751 & 48 & -1.15 \\
\hline 28 & 14 & 7,033 & 3.699 & 0.230 & 0.2524 & 0.0132 & 0.1063 & 0.0029 & 1571 & 50 & 1451 & 68 & 1737 & 50 & 5.16 \\
\hline 29 & 19 & 25,086 & 3.558 & 0.213 & 0.2414 & 0.0123 & 0.1069 & 0.0028 & 1540 & 47 & 1394 & 64 & 1748 & 48 & 6.99 \\
\hline 30 & 17 & 8,185 & 3.441 & 0.206 & 0.2367 & 0.0119 & 0.1055 & 0.0028 & 1514 & 47 & 1369 & 62 & 1722 & 50 & 7.17 \\
\hline 32 & 19 & 13,002 & 3.610 & 0.219 & 0.2462 & 0.0127 & 0.1063 & 0.0028 & 1552 & 48 & 1419 & 66 & 1737 & 48 & 6.04 \\
\hline 33 & 20 & 19,535 & 3.648 & 0.218 & 0.2478 & 0.0126 & 0.1067 & 0.0028 & 1560 & 48 & 1427 & 65 & 1745 & 48 & 5.96 \\
\hline 34 & 19 & 11,165 & 4.408 & 0.278 & 0.3005 & 0.0161 & 0.1064 & 0.0028 & 1714 & 52 & 1694 & 80 & 1738 & 48 & 0.56 \\
\hline 35 & 19 & 4,449 & 3.470 & 0.212 & 0.2391 & 0.0121 & 0.1053 & 0.0030 & 1521 & 48 & 1382 & 63 & 1719 & 52 & 6.70 \\
\hline 36 & 19 & 7,961 & 3.561 & 0.213 & 0.2430 & 0.0123 & 0.1063 & 0.0028 & 1541 & 47 & 1402 & 64 & 1737 & 49 & 6.50 \\
\hline 37 & 19 & 3,838 & 3.522 & 0.219 & 0.2446 & 0.0125 & 0.1045 & 0.0030 & 1532 & 49 & 1410 & 65 & 1705 & 53 & 5.59 \\
\hline 38 & 19 & 5,890 & 3.471 & 0.211 & 0.2371 & 0.0121 & 0.1062 & 0.0029 & 1521 & 48 & 1372 & 63 & 1735 & 50 & 7.39 \\
\hline 39 & 16 & 6,407 & 4.014 & 0.254 & 0.2749 & 0.0147 & 0.1059 & 0.0029 & 1637 & 51 & 1566 & 74 & 1730 & 50 & 2.51 \\
\hline 40 & 19 & 9,895 & 3.977 & 0.242 & 0.2717 & 0.0140 & 0.1062 & 0.0028 & 1630 & 49 & 1549 & 71 & 1735 & 49 & 2.89 \\
\hline 41 & 19 & 6,474 & 4.093 & 0.282 & 0.2800 & 0.0168 & 0.1060 & 0.0029 & 1653 & 56 & 1591 & 85 & 1732 & 50 & 2.07 \\
\hline 44 & 20 & 20,362 & 3.515 & 0.207 & 0.2397 & 0.0120 & 0.1064 & 0.0028 & 1531 & 47 & 1385 & 62 & 1738 & 48 & 7.04 \\
\hline 45 & 19 & 11,621 & 2.633 & 0.179 & 0.1788 & 0.0108 & 0.1068 & 0.0028 & 1310 & 50 & 1060 & 59 & 1746 & 48 & 23.06 \\
\hline 46 & 19 & 13,464 & 3.693 & 0.222 & 0.2516 & 0.0128 & 0.1064 & 0.0028 & 1570 & 48 & 1447 & 66 & 1739 & 49 & 5.32 \\
\hline 47 & 16 & 28,516 & 3.534 & 0.214 & 0.2409 & 0.0123 & 0.1064 & 0.0029 & 1535 & 48 & 1392 & 64 & 1738 & 49 & 6.85 \\
\hline 48 & 18 & 14,739 & 5.985 & 0.417 & 0.4025 & 0.0239 & 0.1078 & 0.0029 & 1974 & 61 & 2181 & 110 & 1763 & 48 & -2.75 \\
\hline 49 & 14 & 4,212 & 3.954 & 0.253 & 0.2710 & 0.0144 & 0.1058 & 0.0030 & 1625 & 52 & 1546 & 73 & 1729 & 52 & 2.87 \\
\hline 50 & 20 & 11,936 & 4.140 & 0.253 & 0.2812 & 0.0145 & 0.1068 & 0.0028 & 1662 & 50 & 1597 & 73 & 1745 & 49 & 2.17 \\
\hline 51 & 19 & 15,852 & 4.203 & 0.274 & 0.2834 & 0.0160 & 0.1076 & 0.0028 & 1675 & 54 & 1608 & 80 & 1758 & 48 & 2.17 \\
\hline 52 & 19 & 37,596 & 4.853 & 0.332 & 0.3285 & 0.0196 & 0.1071 & 0.0028 & 1794 & 58 & 1831 & 95 & 1751 & 48 & -0.84 \\
\hline 53 & 15 & 14,231 & 3.699 & 0.227 & 0.2530 & 0.0132 & 0.1060 & 0.0028 & 1571 & 49 & 1454 & 68 & 1732 & 49 & 5.00 \\
\hline 54 & 20 & 39,189 & 3.679 & 0.220 & 0.2498 & 0.0127 & 0.1068 & 0.0028 & 1567 & 48 & 1437 & 66 & 1746 & 48 & 5.71 \\
\hline 55 & 14 & 13,004 & 3.669 & 0.228 & 0.2512 & 0.0131 & 0.1060 & 0.0029 & 1565 & 50 & 1444 & 68 & 1731 & 51 & 5.21 \\
\hline 56 & 20 & 16,926 & 3.621 & 0.216 & 0.2471 & 0.0124 & 0.1063 & 0.0029 & 1554 & 47 & 1424 & 64 & 1736 & 49 & 5.88 \\
\hline 57 & 19 & 12,072 & 3.761 & 0.230 & 0.2561 & 0.0132 & 0.1065 & 0.0029 & 1584 & 49 & 1470 & 68 & 1740 & 50 & 4.75 \\
\hline 58 & 19 & 15,351 & 4.061 & 0.251 & 0.2762 & 0.0145 & 0.1066 & 0.0028 & 1646 & 50 & 1572 & 73 & 1742 & 49 & 2.58 \\
\hline 59 & 19 & 17,935 & 3.524 & 0.209 & 0.2392 & 0.0120 & 0.1068 & 0.0028 & 1533 & 47 & 1383 & 63 & 1746 & 48 & 7.31 \\
\hline 60 & 19 & 26,793 & 4.350 & 0.271 & 0.2955 & 0.0157 & 0.1068 & 0.0028 & 1703 & 51 & 1669 & 78 & 1745 & 48 & 1.00 \\
\hline 61 & 20 & 24,950 & 4.564 & 0.289 & 0.3095 & 0.0168 & 0.1069 & 0.0028 & 1743 & 53 & 1738 & 83 & 1748 & 48 & 0.12 \\
\hline 62 & 18 & 8,432 & 3.815 & 0.240 & 0.2571 & 0.0138 & 0.1076 & 0.0029 & 1596 & 51 & 1475 & 71 & 1760 & 49 & 5.01 \\
\hline 63 & 20 & 9,142 & 3.420 & 0.203 & 0.2335 & 0.0117 & 0.1062 & 0.0028 & 1509 & 47 & 1353 & 61 & 1736 & 49 & 8.03 \\
\hline 64 & 19 & 25,823 & 5.130 & 0.340 & 0.3498 & 0.0198 & 0.1064 & 0.0028 & 1841 & 56 & 1934 & 95 & 1738 & 48 & -1.78 \\
\hline 65 & 19 & 23,251 & 3.567 & 0.213 & 0.2440 & 0.0123 & 0.1060 & 0.0028 & 1542 & 47 & 1407 & 64 & 1732 & 49 & 6.25 \\
\hline 66 & 19 & 69,362 & 3.603 & 0.213 & 0.2452 & 0.0123 & 0.1066 & 0.0028 & 1550 & 47 & 1414 & 64 & 1741 & 48 & 6.26 \\
\hline 67 & 19 & 40,568 & 3.489 & 0.207 & 0.2375 & 0.0119 & 0.1066 & 0.0028 & 1525 & 47 & 1374 & 62 & 1741 & 49 & 7.47 \\
\hline 68 & 19 & 11,193 & 3.623 & 0.221 & 0.2460 & 0.0124 & 0.1068 & 0.0030 & 1555 & 49 & 1418 & 64 & 1745 & 52 & 6.23 \\
\hline 69 & 16 & 11,107 & 5.416 & 0.368 & 0.3643 & 0.0209 & 0.1078 & 0.0029 & 1887 & 58 & 2003 & 99 & 1763 & 50 & -2.00 \\
\hline 70 & 19 & 18,430 & 4.328 & 0.274 & 0.2948 & 0.0160 & 0.1065 & 0.0028 & 1699 & 52 & 1666 & 80 & 1740 & 48 & 0.99 \\
\hline 71 & 20 & 12,325 & 4.061 & 0.249 & 0.2778 & 0.0144 & 0.1060 & 0.0028 & 1647 & 50 & 1580 & 73 & 1733 & 48 & 2.27 \\
\hline 72 & 18 & 11,081 & 4.359 & 0.273 & 0.2969 & 0.0157 & 0.1065 & 0.0028 & 1705 & 52 & 1676 & 78 & 1740 & 49 & 0.84 \\
\hline 73 & 20 & 21,180 & 4.317 & 0.291 & 0.2930 & 0.0173 & 0.1069 & 0.0028 & 1697 & 56 & 1656 & 86 & 1747 & 48 & 1.21 \\
\hline 74 & 19 & 17,459 & 4.476 & 0.288 & 0.3056 & 0.0169 & 0.1062 & 0.0028 & 1727 & 53 & 1719 & 84 & 1736 & 48 & 0.20 \\
\hline
\end{tabular}


Isotopic ratios (corrected)

Ages (Ma)

\begin{tabular}{|c|c|c|c|c|c|c|c|c|c|c|c|c|c|c|c|}
\hline \# & $\mathrm{N}$ & $\frac{{ }^{206} \mathrm{~Pb}}{{ }^{204} \mathrm{~Pb}^{\dagger}}$ & $\begin{array}{c}{ }^{207} \mathrm{~Pb}^{*} \\
{ }^{235} \mathrm{U} \\
\end{array}$ & $\begin{array}{c} \pm \\
(\mathrm{abs})\end{array}$ & $\begin{array}{c}{ }^{206} \mathrm{~Pb}^{*} \\
{ }^{238} \mathrm{U} \\
\end{array}$ & $\begin{array}{c} \pm \\
\text { (abs) }\end{array}$ & $\begin{array}{c}{ }^{207} \mathrm{~Pb}^{*} \\
{ }^{206} \mathrm{~Pb}^{*}\end{array}$ & $\begin{array}{c} \pm \\
(\mathrm{abs})\end{array}$ & $\begin{array}{c}{ }^{207} \mathrm{~Pb}^{*} \\
{ }^{235} \mathrm{U} \\
\end{array}$ & $\begin{array}{c} \pm \\
(\mathrm{abs})\end{array}$ & $\begin{array}{c}{ }^{206} \mathrm{~Pb}^{*} \\
{ }^{238} \mathrm{U} \\
\end{array}$ & $\begin{array}{c} \pm \\
\pm \\
\text { (abs) }\end{array}$ & $\begin{array}{l}{ }^{207} \mathrm{~Pb}^{*} \\
{ }^{206} \mathrm{~Pb}^{*}\end{array}$ & $\begin{array}{c} \pm \\
(\mathrm{abs})\end{array}$ & $\begin{array}{c}\text { Disc. } \\
(\%)\end{array}$ \\
\hline 75 & 19 & 39,780 & 4.193 & 0.269 & 0.2855 & 0.0159 & 0.1065 & 0.0028 & 1673 & 53 & 1619 & 80 & 1741 & 48 & 1.73 \\
\hline 76 & 14 & 11,530 & 4.077 & 0.257 & 0.2782 & 0.0149 & 0.1063 & 0.0029 & 1650 & 51 & 1582 & 75 & 1736 & 50 & 2.29 \\
\hline 77 & 18 & 28,795 & 4.513 & 0.289 & 0.3063 & 0.0168 & 0.1069 & 0.0028 & 1733 & 53 & 1722 & 83 & 1746 & 48 & 0.29 \\
\hline 78 & 20 & 27,520 & 4.320 & 0.282 & 0.2939 & 0.0166 & 0.1066 & 0.0028 & 1697 & 54 & 1661 & 83 & 1742 & 48 & 1.08 \\
\hline 79 & 18 & 18,618 & 3.952 & 0.243 & 0.2687 & 0.0140 & 0.1067 & 0.0028 & 1624 & 50 & 1534 & 71 & 1744 & 49 & 3.36 \\
\hline 80 & 15 & 14,596 & 5.360 & 0.424 & 0.3670 & 0.0258 & 0.1059 & 0.0029 & 1878 & 68 & 2015 & 121 & 1730 & 50 & -2.32 \\
\hline 81 & 15 & 14,618 & 3.792 & 0.235 & 0.2572 & 0.0135 & 0.1069 & 0.0029 & 1591 & 50 & 1475 & 69 & 1748 & 50 & 4.77 \\
\hline 82 & 20 & 20,834 & 4.009 & 0.244 & 0.2730 & 0.0141 & 0.1065 & 0.0028 & 1636 & 49 & 1556 & 71 & 1741 & 48 & 2.87 \\
\hline 83 & 19 & 9,663 & 4.836 & 0.318 & 0.3295 & 0.0184 & 0.1064 & 0.0029 & 1791 & 55 & 1836 & 89 & 1739 & 49 & -1.01 \\
\hline 84 & 19 & 12,213 & 4.724 & 0.354 & 0.3202 & 0.0214 & 0.1070 & 0.0028 & 1772 & 63 & 1791 & 105 & 1749 & 48 & -0.46 \\
\hline 85 & 15 & 17,605 & 3.592 & 0.220 & 0.2443 & 0.0127 & 0.1066 & 0.0029 & 1548 & 49 & 1409 & 66 & 1742 & 49 & 6.42 \\
\hline 86 & 20 & 16,358 & 3.888 & 0.241 & 0.2635 & 0.0140 & 0.1070 & 0.0028 & 1611 & 50 & 1508 & 71 & 1750 & 48 & 4.03 \\
\hline 87 & 19 & 12,441 & 3.906 & 0.237 & 0.2662 & 0.0138 & 0.1064 & 0.0028 & 1615 & 49 & 1521 & 70 & 1739 & 48 & 3.54 \\
\hline 88 & 10 & 5,689 & 5.631 & 0.460 & 0.3721 & 0.0267 & 0.1098 & 0.0031 & 1921 & 70 & 2039 & 126 & 1795 & 52 & -1.96 \\
\hline 89 & 19 & 14,814 & 4.334 & 0.273 & 0.2958 & 0.0159 & 0.1063 & 0.0028 & 1700 & 52 & 1670 & 79 & 1736 & 49 & 0.87 \\
\hline 90 & 19 & 16,747 & 3.777 & 0.230 & 0.2567 & 0.0133 & 0.1067 & 0.0028 & 1588 & 49 & 1473 & 68 & 1744 & 49 & 4.74 \\
\hline 91 & 15 & 19,910 & 3.936 & 0.390 & 0.2685 & 0.0251 & 0.1063 & 0.0029 & 1621 & 80 & 1533 & 127 & 1737 & 50 & 3.27 \\
\hline 92 & 18 & 14,343 & 4.319 & 0.276 & 0.2901 & 0.0158 & 0.1080 & 0.0029 & 1697 & 53 & 1642 & 79 & 1765 & 49 & 1.70 \\
\hline 93 & 15 & 4,385 & 3.633 & 0.242 & 0.2424 & 0.0140 & 0.1087 & 0.0030 & 1557 & 53 & 1399 & 73 & 1778 & 50 & 7.51 \\
\hline 94 & 19 & 14,142 & 3.623 & 0.216 & 0.2470 & 0.0125 & 0.1064 & 0.0028 & 1555 & 47 & 1423 & 65 & 1738 & 48 & 5.95 \\
\hline 95 & 20 & 9,525 & 3.539 & 0.211 & 0.2430 & 0.0122 & 0.1056 & 0.0028 & 1536 & 47 & 1402 & 63 & 1725 & 49 & 6.24 \\
\hline 96 & 19 & 18,483 & 3.696 & 0.221 & 0.2524 & 0.0128 & 0.1062 & 0.0028 & 1571 & 48 & 1451 & 66 & 1735 & 48 & 5.14 \\
\hline 97 & 19 & 23,377 & 3.506 & 0.208 & 0.2396 & 0.0120 & 0.1061 & 0.0028 & 1529 & 47 & 1384 & 63 & 1734 & 49 & 6.98 \\
\hline 98 & 16 & 12,692 & 3.986 & 0.248 & 0.2715 & 0.0143 & 0.1065 & 0.0029 & 1631 & 50 & 1548 & 73 & 1740 & 49 & 3.01 \\
\hline 99 & 19 & 11,016 & 3.419 & 0.205 & 0.2344 & 0.0117 & 0.1058 & 0.0029 & 1509 & 47 & 1358 & 61 & 1728 & 51 & 7.67 \\
\hline 100 & 19 & 10,480 & 3.446 & 0.208 & 0.2360 & 0.0118 & 0.1059 & 0.0030 & 1515 & 47 & 1366 & 62 & 1730 & 52 & 7.48 \\
\hline 101 & 17 & 12,035 & 3.365 & 0.202 & 0.2285 & 0.0115 & 0.1068 & 0.0029 & 1496 & 47 & 1327 & 60 & 1746 & 50 & 9.17 \\
\hline 102 & 15 & 1,680 & 3.793 & 0.242 & 0.2401 & 0.0126 & 0.1146 & 0.0033 & 1591 & 51 & 1387 & 65 & 1874 & 52 & 10.15 \\
\hline 103 & 20 & 11,394 & 3.717 & 0.226 & 0.2524 & 0.0130 & 0.1068 & 0.0028 & 1575 & 49 & 1451 & 67 & 1746 & 48 & 5.34 \\
\hline 104 & 19 & 19,785 & 3.930 & 0.239 & 0.2672 & 0.0139 & 0.1067 & 0.0028 & 1620 & 49 & 1526 & 70 & 1744 & 48 & 3.52 \\
\hline 105 & 19 & 5,220 & 3.352 & 0.208 & 0.2293 & 0.0116 & 0.1060 & 0.0032 & 1493 & 49 & 1331 & 61 & 1732 & 56 & 8.67 \\
\hline 106 & 19 & 38,184 & 3.475 & 0.206 & 0.2345 & 0.0118 & 0.1075 & 0.0028 & 1522 & 47 & 1358 & 62 & 1757 & 48 & 8.34 \\
\hline 107 & 20 & 27,917 & 3.423 & 0.203 & 0.2330 & 0.0116 & 0.1065 & 0.0029 & 1510 & 47 & 1350 & 61 & 1741 & 50 & 8.22 \\
\hline
\end{tabular}

All errors reported at 2-sigma level unless otherwise indicated; \# = grain identifier; $r$ = rim analysis on grain where core and rim domains were clearly distinguishable; $\mathrm{N}=$ number of analyses used in ratio calculations (max. 20$) ;{ }^{\dagger}={ }^{204} \mathrm{~Pb}$ corrected for Hg interference; * = radiogenic Pb; Disc. (\%) = percent discordance calculated from comparison of ${ }^{207} \mathrm{~Pb} /{ }^{235} \mathrm{U}$ and ${ }^{206} \mathrm{~Pb} /{ }^{235} \mathrm{U}$ ratios. Measured $\mathrm{Pb} / \mathrm{U}$ ratios corrected for $\mathrm{Pb} / \mathrm{U}$ fractionation by comparison with in-house standard zircons. 